Supporting Information for

\title{
A Simple Primary-Tertiary Diamine-Brønsted Acid Catalyst for Asymmetric Direct Aldol Reaction of Linear Aliphatic Ketones
}

Sanzhong Luo, ${ }^{* \dagger}$ Hui Xu ${ }^{\dagger}{ }^{\top}$ Jiuyuan $\mathrm{Li}^{\dagger}{ }^{\dagger}$ Long Zhang ${ }^{\ddagger}$ and Jin-Pei Cheng ${ }^{\dagger \neq}$

Beijing National Laboratory for Molecular Sciences (BNLMS), Center for Molecular Science, Institute of Chemistry and Graduate School, Chinese Academy of Sciences, Beijing, 100080, China and Department of Chemistry, State Key Laboratory of elemento-organic Chemistry, Nankai University, Tianjin, 300071, China.

luosz@iccas.ac.cn, chengjp@mail.most.gov.cn

General informationS2

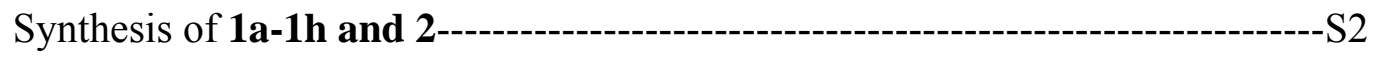

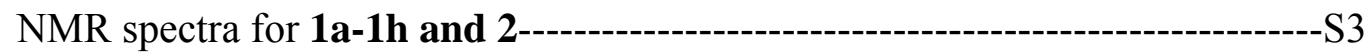

General experimental procedure----------------------------------------------------------S5

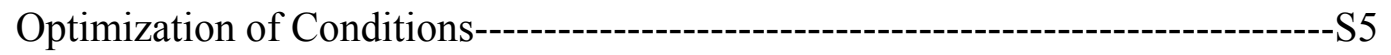

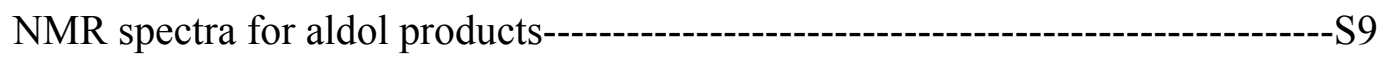

HPLC data--------------------------------------------------------------------------------------S14

NMR spectra for new compounds--------------------------------------------------------S17

HPLC spectra-------------------------------------------------------------------------------S43 
General Information: Commercial reagents were used as received, unless otherwise stated. Chemical shifts are reported in ppm from tetramethylsilane with the solvent resonance as the internal standard. The following abbreviations were used to designate chemical shift mutiplicities: $\mathrm{s}$ $=$ singlet, $\mathrm{d}=$ doublet, $\mathrm{t}=$ triplet, $\mathrm{q}=$ quartet, $\mathrm{h}=$ heptet, $\mathrm{m}=$ multiplet, $\mathrm{br}=$ broad. All first-order splitting patterns were assigned on the basis of the appearance of the multiplet. Splitting patterns that could not be easily interpreted are designated as multiplet (m) or broad (br). Mass spectra were obtained using fast-atom bombard (FAB) spectrometer or electrospray ionization (ESI) mass spectrometer. Optical rotations were measured using a $1 \mathrm{~mL}$ cell with a $1 \mathrm{dm}$ path length on a Perkin-Elmer 341 digital polarimeter and are reported as follows: $[\alpha]_{\mathrm{D}}{ }^{\mathrm{rt}}(c$ in $\mathrm{g}$ per $100 \mathrm{~mL}$ of solvent). HPLC analysis was performed using ChiralPak columns purchased.

\section{Representative procedure for the synthesis of catalysts:}

Synthesis of catalyst $\mathbf{1 d}$ :

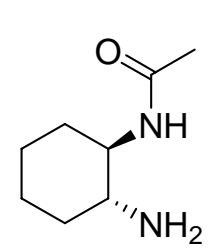

$1 \mathbf{a}$

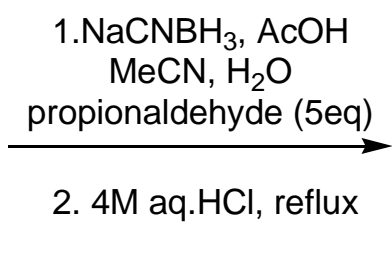

2. $4 \mathrm{M}$ aq. $\mathrm{HCl}$, reflux

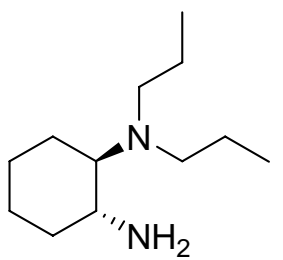

1d

1a [T. L. 2000, 41, 8431-8434 ] (3.40 g, $21.8 \mathrm{mmol}, 1 \mathrm{eq})$, propionaldehyde (7.68 mL, $109.0 \mathrm{mmol}$, 5 eq), and $\mathrm{H}_{2} \mathrm{O}(6 \mathrm{~mL})$ were mixed in a $100-\mathrm{mL}$ round bottomed flask with acetonitrile $(120 \mathrm{~mL})$ and stirred for 15 minutes. Sodium cyanoborohydride $(2.87 \mathrm{~g}, 45.8 \mathrm{mmol}, 2.1 \mathrm{eq})$ was added followed by $\mathrm{AcOH}(6 \mathrm{~mL}) 15$ minutes later. After 2 hours, the volatiles were removed in vacuo and the resulting residue transferred to a separatory funnel with ethyl acetate $(200 \mathrm{~mL})$ and $1 \mathrm{M} \mathrm{NaOH}$ $(50 \mathrm{~mL})$. The organic layer was washed twice with $1 \mathrm{M} \mathrm{NaOH}(50 \mathrm{~mL})$, brine $(50 \mathrm{~mL})$, and dried $\left(\mathrm{Na}_{2} \mathrm{SO}_{4}\right)$. The ethyl acetate was removed in vacuo and the crude residue was taken up in $4 \mathrm{M} \mathrm{HCl}$ $(100 \mathrm{~mL})$. The resulting solution was refluxed for 12 hours and after cooling to ambient temperature was made basic to $\mathrm{pH} \approx 13$ with $4 \mathrm{M} \mathrm{NaOH}$. The aqueous layer was extracted three 
times with $\mathrm{CH}_{2} \mathrm{Cl}_{2}(100 \mathrm{~mL})$, and dried $\left(\mathrm{Na}_{2} \mathrm{SO}_{4}\right)$. Solvent removal in vacuo afforded crude $\mathbf{1 d}$ as a pale yellow oil which was distilled in vacuo to afford pure 1d (3.2 g, 74\% yield over all steps) as a colorless oil. $[\alpha]^{20}{ }_{\mathrm{D}}=-137.0 \quad\left(\mathrm{c}=1.0, \mathrm{CHCl}_{3}\right) ;{ }^{1} \mathrm{H}$ NMR $\left(300 \mathrm{MHz}, \mathrm{CDCl}_{3}\right): \delta 0.82(6 \mathrm{H}, \mathrm{t}, J=7.2$ $\mathrm{Hz}), 0.96-1.10(4 \mathrm{H}, \mathrm{m}), 1.23-1.38(4 \mathrm{H}, \mathrm{m}), 1.45-1.48(1 \mathrm{H}, \mathrm{m}), 1.69-1.71(4 \mathrm{H}, \mathrm{m}), 1.87-2.06(2 \mathrm{H}$, m), 2.17-2.41 (4H, m), 2.43-2.55 (1H, m); ${ }^{13} \mathrm{C} \mathrm{NMR}\left(\mathrm{CDCl}_{3}, 75 \mathrm{MHz}\right): \delta 11.8,22.5,22.8,25.1$, 26.0, 35.1, 51.3, 52.0, 66.9, 76.6, 77.1, 77.5; HRMS for $\mathrm{C}_{12} \mathrm{H}_{26} \mathrm{~N}_{2}$ (M), calcd. 198.2096, found 198.2098.

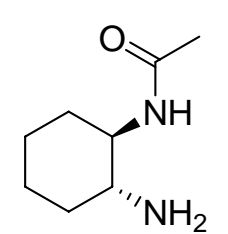

$1 a$

The title product was prepared according to the procedure of Finney ${ }^{1}$ as yellow solid. $[\alpha]^{20}{ }_{\mathrm{D}}=+10.8\left(\mathrm{c}=1.0, \mathrm{CHCl}_{3}\right) ;{ }^{1} \mathrm{H} \mathrm{NMR}\left(300 \mathrm{MHz}, \mathrm{CDCl}_{3}\right): \delta 1.02-1.25(4 \mathrm{H}$, m), 1.61-1.64 (2H, m), $1.72(3 \mathrm{H}, \mathrm{s}), 1.90-1.92(4 \mathrm{H}, \mathrm{m}), 2.28-2.35(1 \mathrm{H}, \mathrm{m}), 3.32-3.42$ $(1 \mathrm{H}, \mathrm{m}), 6.24(1 \mathrm{H}, \mathrm{br}) ;{ }^{13} \mathrm{C} \mathrm{NMR}\left(\mathrm{CDCl}_{3}, 75 \mathrm{MHz}\right): \delta 23.4,25.0,32.4,35.4,55.2$, 56.0, 57.6, 170.5; HRMS for $\mathrm{C}_{8} \mathrm{H}_{17} \mathrm{~N}_{2} \mathrm{O}(\mathrm{M}+1)^{+}$, calcd. 157.1335, found 157.1336.

$\overbrace{{ }^{\prime \prime} \mathrm{NH}_{2}}^{\mathrm{N}}$

The title product was prepared according to the general procedure as colorless oil in $70 \%$ yield. $[\alpha]^{20}=-29.7\left(\mathrm{c}=1.0, \mathrm{CHCl}_{3}\right) ;{ }^{1} \mathrm{H} \mathrm{NMR}\left(300 \mathrm{MHz}, \mathrm{CDCl}_{3}\right): \delta 1.10-1.18$ $(4 \mathrm{H}, \mathrm{m}), 1.72-1.77(4 \mathrm{H}, \mathrm{m}), 1.88-2.00(2 \mathrm{H}, \mathrm{m}), 2.22(6 \mathrm{H}, \mathrm{s}), 2.50-2.56(1 \mathrm{H}, \mathrm{m}) ;{ }^{13} \mathrm{C}$ NMR $\left(\mathrm{CDCl}_{3}, 75 \mathrm{MHz}\right): \delta 20.7,25.1,25.7,35.3,40.2,51.5,69.9 ; \mathrm{HRMS}$ for $\mathrm{C}_{8} \mathrm{H}_{18} \mathrm{~N}_{2}$ (M), calcd. 142.1472, found 142.1470.

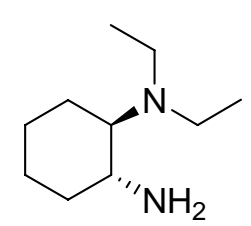

$1 \mathrm{c}$

The title product was prepared according to the general procedure as colorless oil in $85 \%$ yield. $[\alpha]_{\mathrm{D}}^{20}=-112.9\left(\mathrm{c}=1.0, \mathrm{CHCl}_{3}\right) ;{ }^{1} \mathrm{H}$ NMR $\left(300 \mathrm{MHz}, \mathrm{CDCl}_{3}\right): \delta$ 0.91-1.00 (6H, m), 1.00-1.10 (3H, m), 1.62-1.70 (6H, m), 1.86-2.14 (2H, m), 2.27-2.30 (2H, m), 2.49-2.56 (3H, m); ${ }^{13} \mathrm{C} \mathrm{NMR}\left(\mathrm{CDCl}_{3}, 75 \mathrm{MHz}\right): \delta 15.0,23.1$, 25.1, 26.1, 35.2, 43.4, 51.2, 66.4; HRMS for $\mathrm{C}_{10} \mathrm{H}_{22} \mathrm{~N}_{2}$ (M), calcd. 170.1783, found 170.1785.

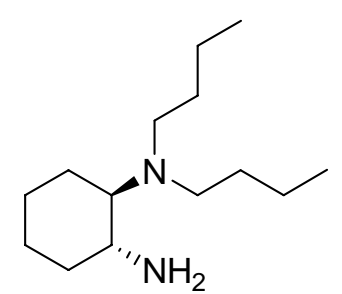

$1 e$
The title product was prepared according to the general procedure as colorless oil in $83 \%$ yield. $[\alpha]^{20}{ }_{\mathrm{D}}=-108.6\left(\mathrm{c}=1.0, \mathrm{CHCl}_{3}\right) ;{ }^{1} \mathrm{H}$ NMR $(300 \mathrm{MHz}$, 
$\left.\mathrm{CDCl}_{3}\right): \delta$ 0.80-0.85 (6H, m), 1.07-1.33 (12H, m), 1.60-1.70 (5H, m), 1.82-2.00 (2H, m), 2.20-2.22 $(2 \mathrm{H}, \mathrm{m}), 2.31-2.38(3 \mathrm{H}, \mathrm{m}) ;{ }^{13} \mathrm{C} \mathrm{NMR}\left(\mathrm{CDCl}_{3}, 75 \mathrm{MHz}\right): \delta 14.0,20.6,22.8,25.1,26.0,31.7,35.2$, 49.8, 51.3, 66.8; HRMS for $\mathrm{C}_{14} \mathrm{H}_{31} \mathrm{~N}_{2}(\mathrm{M}+1)^{+}$, calcd. 227.2482, found 198.224.2479.

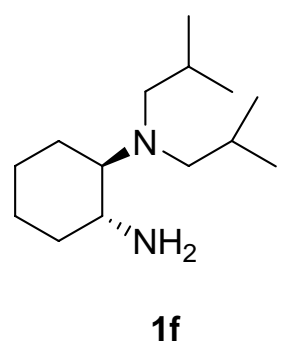

The title product was prepared according to the general procedure as colorless oil in $63 \%$ yield. $[\alpha]^{20}{ }_{\mathrm{D}}=-148.1\left(\mathrm{c}=1.0, \mathrm{CHCl}_{3}\right) ;{ }^{1} \mathrm{H} \mathrm{NMR}\left(300 \mathrm{MHz}, \mathrm{CDCl}_{3}\right): \delta$ 0.77-0.91 (12H, m), 1.00-1.29 (4H, m), 1.60-1.80 (7H, m), 1.88-2.11 (4H, m), 2.11-2.20 (2H, m), 2.48-2.57 (1H, m); ${ }^{13} \mathrm{C} \mathrm{NMR}\left(\mathrm{CDCl}_{3}, 75 \mathrm{MHz}\right): \delta 20.8,21.2$, 22.1, 25.2, 26.0, 26.7, 35.3, 51.2, 66.9; HRMS for $\mathrm{C}_{14} \mathrm{H}_{30} \mathrm{~N}_{2}(\mathrm{M})$, calcd. 226.2409, found 198.226.2407.

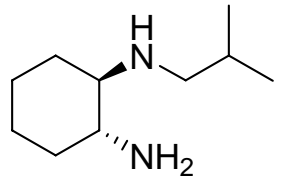

The title product was prepared according to the general procedure as colorless oil in $23 \%$ yield. $[\alpha]_{\mathrm{D}}^{20}=-83.3\left(\mathrm{c}=1.0, \mathrm{CHCl}_{3}\right) ;{ }^{1} \mathrm{H} \mathrm{NMR}\left(300 \mathrm{MHz}, \mathrm{CDCl}_{3}\right): \delta$ 0.85-0.87 (6H, dd, $J=1.3 \mathrm{~Hz}, 1.3 \mathrm{~Hz}, 6.6 \mathrm{~Hz}), 1.00-1.21(4 \mathrm{H}, \mathrm{m}), 1.30-1.50(1 \mathrm{H}$, 19 br), 1.61-1.68 (4H, m), 1.78-1.88 (1H, m), 1.88-2.00 (2H, m), 2.20-2.26 (2H, m), 2.49-2.53 (1H, m); ${ }^{13} \mathrm{C} \mathrm{NMR}\left(\mathrm{CDCl}_{3}, 75 \mathrm{MHz}\right): \delta 20.6,20.7,25.3,25.4,28.8,31.6,36.0,55.2,55.4$, 64.1; HRMS for $\mathrm{C}_{10} \mathrm{H}_{22} \mathrm{~N}_{2}(\mathrm{M})$, calcd. 170.1783, found 170.1785 .

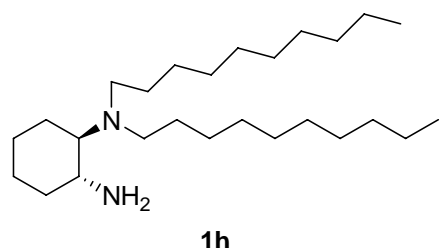

The title product was prepared according to the general procedure as colorless oil in $87 \%$ yield. [ $\alpha]_{\mathrm{D}}^{20}=-59.3\left(\mathrm{c}=1.0, \mathrm{CHCl}_{3}\right) ;{ }^{1} \mathrm{H} \mathrm{NMR}$ $\left(300 \mathrm{MHz}, \mathrm{CDCl}_{3}\right): \delta 0.83-0.88(6 \mathrm{H}, \mathrm{m}), 1.24(36 \mathrm{H}$, br s), $1.50-1.59$ $(1 \mathrm{H}, \mathrm{m}), 1.59-1.68(1 \mathrm{H}, \mathrm{m}), 1.68-1.80(3 \mathrm{H}, \mathrm{m}), 1.80-2.17(3 \mathrm{H}, \mathrm{m})$, 2.17-2.35 (2H, m), 2.35-2.50 (3H, m); ${ }^{13} \mathrm{C} \mathrm{NMR}\left(\mathrm{CDCl}_{3}, 75 \mathrm{MHz}\right): \delta 14.1,22.7,22.8,25.2,26.1$, 27.5, 29.4, 29.5, 29.6, 29.7, 31.9, 35.1, 50.1, 51.3, 66.9; HRMS for $\mathrm{C}_{26} \mathrm{H}_{54} \mathrm{~N}_{2}(\mathrm{M})$, calcd. 394.4287, found 394.4291.<smiles>CN(C)[C@@H](c1ccccc1)[C@H](N)c1ccccc1</smiles>

2
The title product was prepared according to the general procedure as pale yellow oil in $40 \%$ yield. $[\alpha]^{20}{ }_{\mathrm{D}}=86.4\left(\mathrm{c}=0.5, \mathrm{CHCl}_{3}\right) ;{ }^{1} \mathrm{H}$ NMR $\left(300 \mathrm{MHz}, \mathrm{CDCl}_{3}\right): \delta$ 2.08-2.13 (2H, br), $2.20(6 \mathrm{H}, \mathrm{s}), 3.62(1 \mathrm{H}, \mathrm{d}, J=10.6 \mathrm{~Hz}), 4.41(1 \mathrm{H}, \mathrm{d}, J=10.4$ 
$\mathrm{Hz}), 6.93-7.04(3 \mathrm{H}, \mathrm{m}), 7.04-7.13(5 \mathrm{H}, \mathrm{m}), 7.18-7.25(2 \mathrm{H}, \mathrm{d}, J=7.9 \mathrm{~Hz}) ;{ }^{13} \mathrm{C} \mathrm{NMR}\left(\mathrm{CDCl}_{3}\right.$, 75MHz): $\delta 41.0,55.7,75.4,126.8,126.9,127.3,128.0,128.1,129.8,134.0,143.4$; HRMS for $\mathrm{C}_{16} \mathrm{H}_{20} \mathrm{~N}_{2}(\mathrm{M})$, calcd. 240.1626, found 240.1628.

\section{General procedure for aldol reaction:}

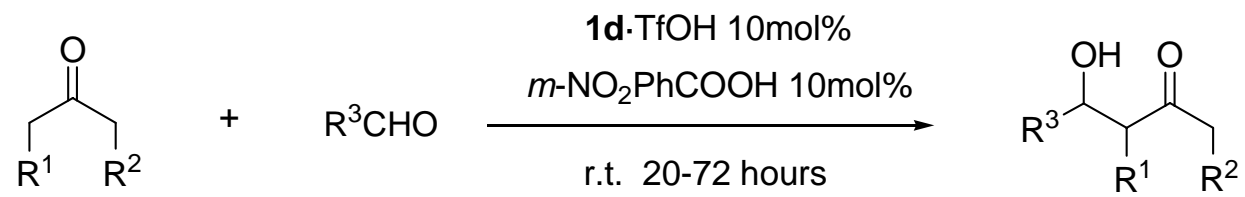

To a given anhydrous ketone (20. equiv.) was added the corresponding aldehyde $(0.25 \mathrm{mmol})$, 3-nitrobenzoic acid $(0.025 \mathrm{mmol})$ and catalyst $\mathbf{1} \mathrm{d} \cdot \mathbf{T f O H}(0.025 \mathrm{mmol})$ (prepared in $\mathrm{CH}_{2} \mathrm{Cl}_{2}$ with 1 eq. 1d and 1 eq. TfOH). The resulting mixture was stirred under room temperature for 20-72 hours and then directly purified through flash column chromatography on a silica gel to afford the pure products.

Products $3 \mathbf{a}-\mathbf{3 h}{ }^{2}$ and $\mathbf{4 a - 4 b}{ }^{2 \mathrm{c}, 2 \mathrm{~d}, 3}, \mathbf{4 e}$ (anti isomer) ${ }^{4}$ and $\mathbf{4} \mathbf{m}^{2}$ are known compounds. $\mathbf{4 g}-\mathbf{4 i}$ could be transformed into known compound by simple deprotection of benzyl group. ${ }^{5}$

\section{Optimization of Conditions:}

The first Acid screening:

\begin{tabular}{ccc} 
Yield (\%) ${ }^{\mathrm{a}}$ & Ee (\%) \\
\hline Acid & 86 & 70 \\
$\mathrm{CF}_{3} \mathrm{SO}_{3} \mathrm{H}$ & 47 & 69 \\
$\mathrm{TFA}$ & 9 & 43 \\
$\mathrm{AcOH}$ & 16 & 59 \\
$m-\mathrm{NO}_{2} \mathrm{PhCOOH}$ & 33 & 63 \\
$\mathrm{PTSA}$ & 34 & 60 \\
$\mathrm{Camphor} \mathrm{sulfonic} \mathrm{acid}$ & 13 & 41 \\
$\mathrm{PhCOOH}_{\mathrm{Ch}}$ & 36 & 62
\end{tabular}

a isolated yields 
Solvent screening:

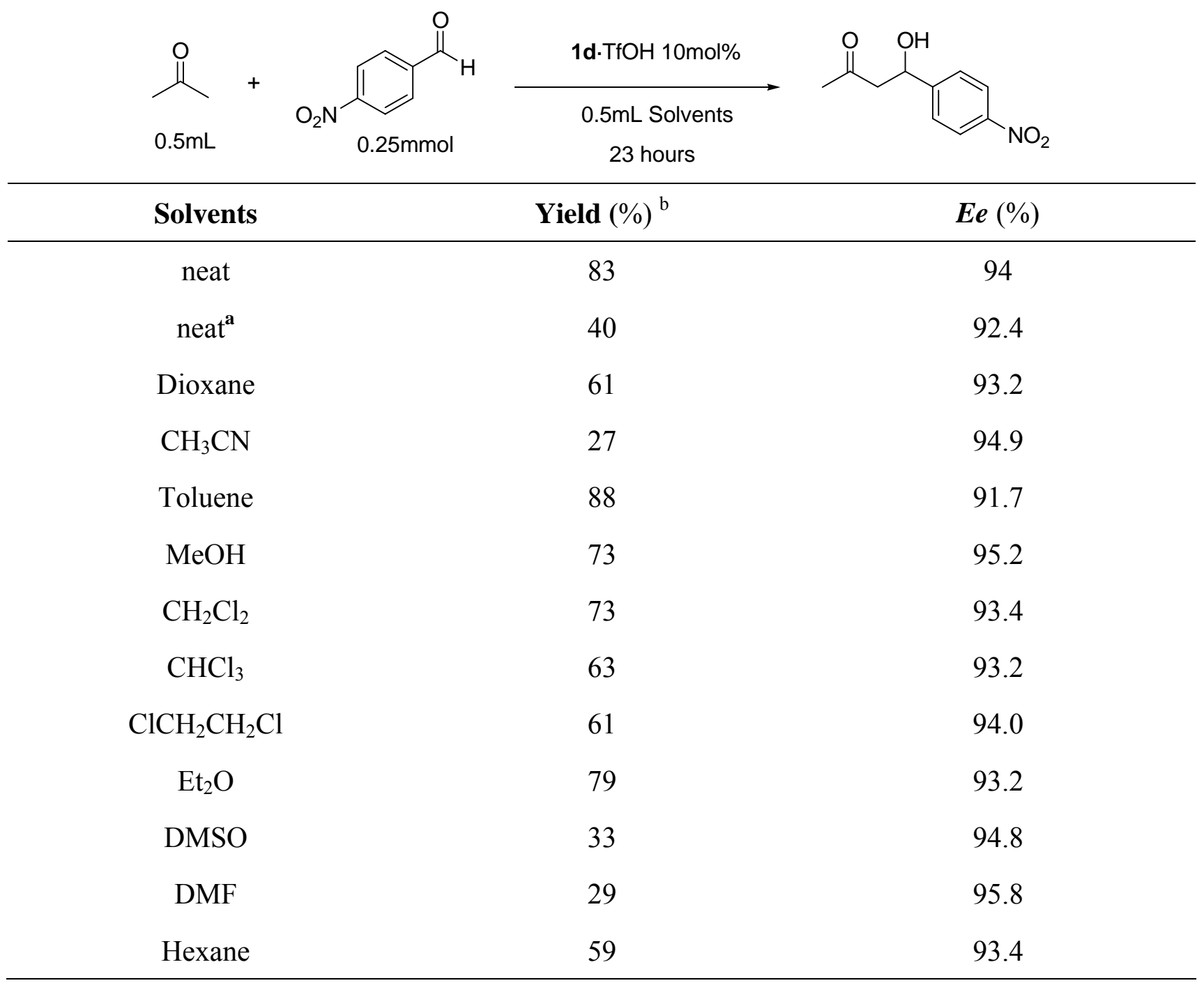

${ }^{a} 10$ eq $\mathrm{H}_{2} \mathrm{O}$ was added. ${ }^{\mathrm{b}}$ isolated yields. 
Screening of the second acid:

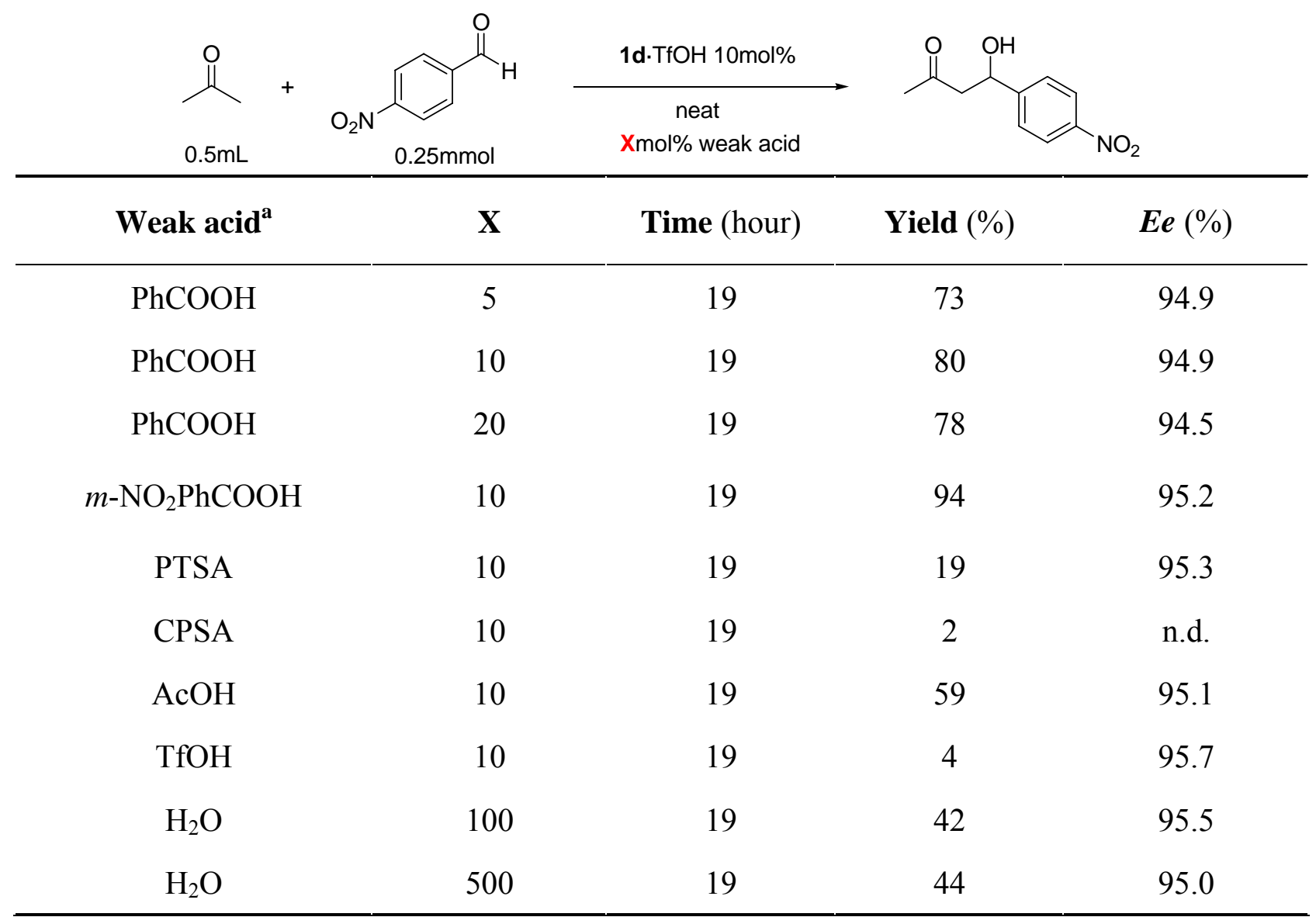

a: PTSA: p-Toluenesulfonic acid; CPSA: D-Camphor-10-Sulfonic Acid. 


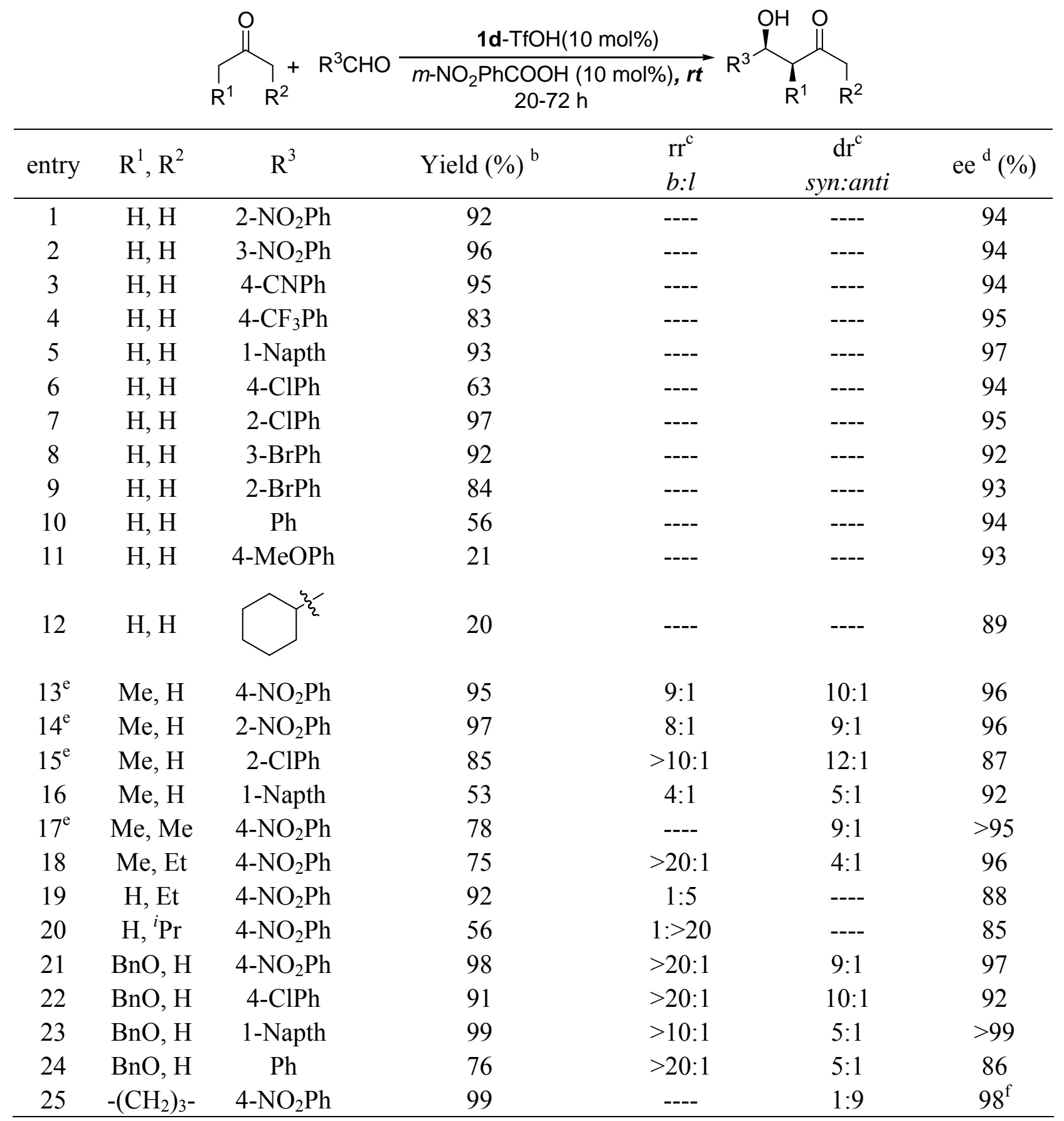

${ }^{\mathrm{a}}$ Reaction with 20 equiv. of ketones under neat conditions. ${ }^{\mathrm{b}}$ Isolated yields. ${ }^{\mathrm{c}}$ Determined by ${ }^{1} \mathrm{H}$ NMR, rr: regioisomer ratio; b:l: the ratio of branched and linear products. ${ }^{\mathrm{d}}$ Determined by HPLC. ${ }^{\mathrm{e}}$ Reactions under $4{ }^{\circ} \mathrm{C}$ with $20 \mathrm{~mol} \%$ of catalyst. ${ }^{\mathrm{f}}$ ee of the anti isomer, reaction with 2.0 equiv. of cyclohexanone in $\mathrm{CH}_{2} \mathrm{Cl}_{2}$ in $12 \mathrm{~h}$. 


\section{Spectra data for all the Aldol products}

Yield: 94\%; Enantiomeric excess: 95\%; $[\alpha]^{20}{ }_{\mathrm{D}}=-61.8\left(\mathrm{c}=1.0, \mathrm{CHCl}_{3}\right)$;<smiles>CC(=O)CC(O)c1ccc([N+](=O)[O-])cc1</smiles>

3a

${ }^{1} \mathrm{H}$ NMR (300 MHz, $\left.\mathrm{CDCl}_{3}\right): \delta 2.22(3 \mathrm{H}, \mathrm{s}), 2.83-2.92(2 \mathrm{H}, \mathrm{m}), 3.56(2 \mathrm{H}, \mathrm{d}$, $J=3.2 \mathrm{~Hz}), 5.24-5.29(1 \mathrm{H}, \mathrm{m}), 7.53(2 \mathrm{H}, \mathrm{d}, J=8.9 \mathrm{~Hz}), 8.22(2 \mathrm{H}, \mathrm{d}, J=$ $8.9 \mathrm{~Hz})$.<smiles>CC(=O)CC(O)c1cccc([N+](=O)[O-])c1</smiles>

3b

Yield: 96\%; Enantiomeric excess: 94\%; $[\alpha]^{20}{ }_{\mathrm{D}}=-66.1\left(\mathrm{c}=2.0, \mathrm{CHCl}_{3}\right)$; ${ }^{1} \mathrm{H}$ NMR $\left(300 \mathrm{MHz} \mathrm{CDCl}_{3}\right): \delta 2.23(3 \mathrm{H}, \mathrm{s}), 2.86-2.96(2 \mathrm{H}, \mathrm{m}), 3.58(1 \mathrm{H}$, br, s), 5.24-5.26 (1H, m), 7.50-7.57 (1H, t, $J=8.1 \mathrm{~Hz}), 7.70-7.76(1 \mathrm{H}, \mathrm{m})$, 8.12-8.24 (2H, m).<smiles>CC(=O)CC(O)c1ccc(C#N)cc1</smiles>

3c

Yield: 95\%; Enantiomeric excess: 94\%; $[\alpha]^{20}{ }_{\mathrm{D}}=-70.2\left(\mathrm{c}=0.5, \mathrm{CHCl}_{3}\right) ;{ }^{1} \mathrm{H}$ NMR (300 MHz, $\left.\mathrm{CDCl}_{3}\right): \delta 2.19(3 \mathrm{H}, \mathrm{s}), 2.81(2 \mathrm{H}, \mathrm{d}, J=6.2 \mathrm{~Hz}), 3.62-3.64$ (1H, m), 5.16-5.21 (1H, m), 7.50(2H, d, $J=7.7 \mathrm{~Hz}), 7.60(2 \mathrm{H}, \mathrm{d}, J=8.3$ $\mathrm{Hz})$.<smiles>CC(=O)CC(O)c1cccc2ccccc12</smiles>

3d<smiles>CC(=O)CC(O)c1ccccc1Cl</smiles>

$3 e$

Yield: 93\%; Enantiomeric excess: 97\%; $[\alpha]^{20}{ }_{\mathrm{D}}=-84.0\left(\mathrm{c}=0.5, \mathrm{CHCl}_{3}\right) ;{ }^{1} \mathrm{H}$ NMR $\left(300 \mathrm{MHz}, \mathrm{CDCl}_{3}\right): \delta 2.23(3 \mathrm{H}, \mathrm{s}), 2.97-3.04(2 \mathrm{H}, \mathrm{m}), 3.38(1 \mathrm{H}, \mathrm{br})$, 5.94-5.98 (1H, m), 7.46-7.52 (3H, m), $7.69(1 \mathrm{H}, \mathrm{d}, J=7.2 \mathrm{~Hz}), 7.79(1 \mathrm{H}, \mathrm{d}, J=$ 8.3 Hz), 7.86-7.89 (1H, m), 8.01-8.02 (1H, m).

Yield: 97\%; Enantiomeric excess: 95\%; $[\alpha]^{20}{ }_{\mathrm{D}}=-86.5\left(\mathrm{c}=2.0, \mathrm{CHCl}_{3}\right) ;{ }^{1} \mathrm{H}$ NMR (300 MHz, $\left.\mathrm{CDCl}_{3}\right): \delta 2.17(3 \mathrm{H}, \mathrm{s}), 2.56-2.63(1 \mathrm{H}, \mathrm{dd}, J=9.6 \mathrm{~Hz}, 9.6 \mathrm{~Hz}$, $17.7 \mathrm{~Hz}), 2.90-2.97(1 \mathrm{H}, \mathrm{dd}, J=2.3 \mathrm{~Hz}, 2.3 \mathrm{~Hz}, 17.7 \mathrm{~Hz}), 3.55(1 \mathrm{H}, \mathrm{d}, J=3.4$ $\mathrm{Hz}), 5.42-5.48(1 \mathrm{H}, \mathrm{m}), 7.12-7.29(3 \mathrm{H}, \mathrm{m}), 7.58-7.59(1 \mathrm{H}, \mathrm{m})$.<smiles>CC(=O)CC(O)c1cccc(Br)c1</smiles>

Yield: 92\%; Enantiomeric excess: 92\%; $[\alpha]^{20}{ }_{\mathrm{D}}=-51.7\left(\mathrm{c}=1.0, \mathrm{CHCl}_{3}\right) ;{ }^{1} \mathrm{H}$ NMR (300 MHz, $\left.\mathrm{CDCl}_{3}\right): \delta 2.20(3 \mathrm{H}, \mathrm{s}), 2.82(1 \mathrm{H}, \mathrm{s}), 2.82-2.86(1 \mathrm{H}, \mathrm{d}, J=2.6$ $\mathrm{Hz}), 3.35-3.41(1 \mathrm{H}, \mathrm{m})$, 5.06-5.16 (1H, m), 7.16-7.31 (2H, m), 7.37-7.44 (1H, m), 
$7.53(1 \mathrm{H}, \mathrm{s})$.<smiles>CC(=O)CC(O)c1ccccc1</smiles>

$3 g$

Yield: 56\%; Enantiomeric excess: 94\%; $[\alpha]^{20}{ }_{\mathrm{D}}=-62.9\left(\mathrm{c}=1.0, \mathrm{CHCl}_{3}\right) ;{ }^{1} \mathrm{H}$ NMR $\left(300 \mathrm{MHz}, \mathrm{CDCl}_{3}\right): \delta 2.20(3 \mathrm{H}, \mathrm{s}), 2.82-2.91(2 \mathrm{H}, \mathrm{m}), 3.25(1 \mathrm{H}, \mathrm{br})$, 5.14-5.18 (1H, dd, $J=3.8 \mathrm{~Hz}, 3.6 \mathrm{~Hz}, 8.7 \mathrm{~Hz}), 7.28-7.33(1 \mathrm{H}, \mathrm{m}), 7.34-7.36(4 \mathrm{H}$, d, $J=4.2 \mathrm{~Hz})$.<smiles>COc1ccc(C(O)CC(C)=O)cc1</smiles>

3h

Yield: 21\%; Enantiomeric excess: 93\%; ${ }^{1} \mathrm{H}$ NMR (300 $\mathrm{MHz}, \mathrm{CDCl}_{3}$ ): $\delta 2.17$ $(3 \mathrm{H}, \mathrm{s}), 2.75-2.92(2 \mathrm{H}, \mathrm{m}), 3.22(1 \mathrm{H}, \mathrm{br}), 3.79(3 \mathrm{H}, \mathrm{s}), 5.07-5.11(1 \mathrm{H}, \mathrm{dd}, J=$ 3.4 Hz, 3.4 Hz, 8.6 Hz), 6.85-6.89 (2H, m), 7.24-7.28 (2H, m).<smiles>CC(=O)[C@H](C)[C@@H](O)c1ccc([N+](=O)[O-])cc1</smiles>

$4 a$

Yield: 95\%; For syn isomer: enantiomeric excess : 96\%; $[\alpha]_{\mathrm{D}}^{20}=-50.6$ $\left(\mathrm{c}=1.0, \mathrm{CHCl}_{3}\right) ;{ }^{1} \mathrm{H} \mathrm{NMR}\left(300 \mathrm{MHz}, \mathrm{CDCl}_{3}\right): \delta 1.04(3 \mathrm{H}, \mathrm{d}, J=7.4 \mathrm{~Hz})$, $2.24(3 \mathrm{H}, \mathrm{s}), 2.78-2.87(1 \mathrm{H}, \mathrm{m}), 3.43(1 \mathrm{H}, \mathrm{d}, J=2.6 \mathrm{~Hz}), 5.28(1 \mathrm{H}, \mathrm{t}, J=$ $2.5 \mathrm{~Hz}), 7.52(2 \mathrm{H}, \mathrm{d}, J=9.0 \mathrm{~Hz}), 8.20(2 \mathrm{H}, \mathrm{d}, J=8.7 \mathrm{~Hz}) ;{ }^{13} \mathrm{C} \mathrm{NMR}$ $\left(\mathrm{CDCl}_{3}, 75 \mathrm{MHz}\right): \delta 9.5,29.1,52.4,71.7,123.5,126.7,147.2,149.0,213.2 ;$ HRMS for $\mathrm{C}_{11} \mathrm{H}_{14} \mathrm{~N}_{2} \mathrm{O}_{4}$ $(\mathrm{M}+1)^{+}$, calcd. 224.0917, found 224.0918.<smiles>CC(=O)[C@H](O)[C@H](O)c1ccccc1[N+]([O-])([O-])[O-]</smiles>

4b

Yield: 97\%; For syn isomer: enantiomeric excess : 96\%; $[\alpha]_{\mathrm{D}}^{20}=+45.6(\mathrm{c}=$ 0.25, $\left.\mathrm{CHCl}_{3}\right) ;{ }^{1} \mathrm{H} \mathrm{NMR}\left(300 \mathrm{MHz}, \mathrm{CDCl}_{3}\right): \delta 1.05(3 \mathrm{H}, \mathrm{d}, J=7.5 \mathrm{~Hz}), 2.30(3 \mathrm{H}$, s), 3.03-3.12 (1H, m), $5.78(1 \mathrm{H}, \mathrm{d}, J=1.9 \mathrm{~Hz}), 7.41-7.51(1 \mathrm{H}, \mathrm{m}), 7.61-7.75(1 \mathrm{H}$, m), $7.88(1 \mathrm{H}, \mathrm{d}, J=7.9 \mathrm{~Hz}), 8.04(1 \mathrm{H}, \mathrm{dd}, J=1.1 \mathrm{~Hz}, 1.1 \mathrm{~Hz}, 8.3 \mathrm{~Hz}) ;{ }^{13} \mathrm{C} \mathrm{NMR}$ $\left(\mathrm{CDCl}_{3}, 75 \mathrm{MHz}\right): \delta 9.2,28.9,49.9,67.9,124.8,128.2,129.4,133.4,137.0,147.1,213.9$; HRMS for $\mathrm{C}_{11} \mathrm{H}_{14} \mathrm{~N}_{2} \mathrm{O}_{4}(\mathrm{M}+1)^{+}$, calcd. 224.0917, found 224.0917.<smiles>CC(=O)C(C)C(O)c1ccccc1Cl</smiles>

Yield: 85\%; For syn isomer: enantiomeric excess : 87\%; Product containing inseparable linear isomer. ${ }^{1} \mathrm{H} \mathrm{NMR}\left(300 \mathrm{MHz}, \mathrm{CDCl}_{3}\right): \delta 0.94-0.99(3 \mathrm{H}, \mathrm{d}, J=$ $7.4 \mathrm{~Hz}), 2.24(3 \mathrm{H}, \mathrm{s}), 2.93-3.06(1 \mathrm{H}, \mathrm{m}), 3.25(1 \mathrm{H}, \mathrm{d}, J=2.6 \mathrm{~Hz}), 5.44-5.49(1 \mathrm{H}$, $\mathrm{m}), 7.14-7.34(3 \mathrm{H}, \mathrm{m}), 7.51-7.62(1 \mathrm{H}, \mathrm{m}) ;{ }^{13} \mathrm{C} \mathrm{NMR}\left(\mathrm{CDCl}_{3}, 75 \mathrm{MHz}\right): \delta 8.8$, 29.0, 49.1, 69.2, 126.7, 128.3, 128.4, 129.3, 131.0, 138.6, 213.9; HRMS for $\mathrm{C}_{11} \mathrm{H}_{14} \mathrm{ClO}_{2}(\mathrm{M}+1)^{+}$, calcd. 213.0677, found 213.0678. 
$\underbrace{1}_{4 d}$

Yield: 53\%; For syn isomer: enantiomeric excess: $92 \%$; $[\alpha]^{20}{ }_{\mathrm{D}}=-66.0(\mathrm{c}=0.5$, $\left.\mathrm{CHCl}_{3}\right) ;{ }^{1} \mathrm{H} \mathrm{NMR}\left(300 \mathrm{MHz}, \mathrm{CDCl}_{3}\right): \delta 1.05-1.12(3 \mathrm{H}, \mathrm{m}), 2.25(3 \mathrm{H}, \mathrm{s})$, 2.98-3.07 (1H, m), $3.17(1 \mathrm{H}, \mathrm{d}, J=2.5 \mathrm{~Hz}), 5.99(1 \mathrm{H}, \mathrm{s}), 7.49-7.54(3 \mathrm{H}, \mathrm{m})$, 7.71-7.76 (1H, d, $J=7.2 \mathrm{~Hz}), 7.76-7.84(1 \mathrm{H}, \mathrm{d}, J=8.1 \mathrm{~Hz}), 7.87-7.90$ (2H, m) ;

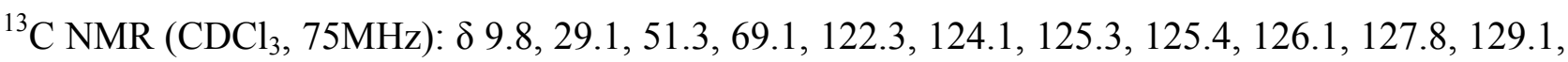

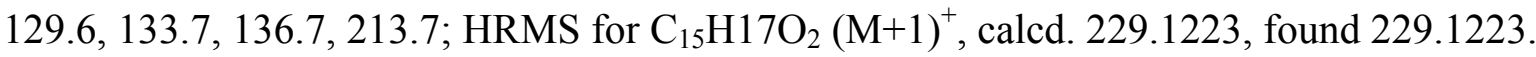<smiles>CCC(=O)[C@H](C)[C@H](O)c1ccc([N+](=O)[O-])cc1</smiles>

Yield: 78\%; For syn isomer: enantiomeric excess : $>95 \%$; $[\alpha]^{20}{ }_{\mathrm{D}}=-19.4$ $\left(\mathrm{c}=1.0, \mathrm{CHCl}_{3}\right) ;{ }^{1} \mathrm{H}$ NMR $\left(300 \mathrm{MHz}, \mathrm{CDCl}_{3}\right): \delta$ 1.01-1.09 $(6 \mathrm{H}, \mathrm{m})$, 2.41-2.65 (2H, m), 2.82-2.85 (1H, m), $3.56(1 \mathrm{H}, \mathrm{d}, J=2.3 \mathrm{~Hz}), 5.23(1 \mathrm{H}, \mathrm{t}$, $J=2.5 \mathrm{~Hz}), 7.50(2 \mathrm{H}, \mathrm{d}, J=8.3 \mathrm{~Hz}), 8.18-8.22(2 \mathrm{H}, \mathrm{m}) ;{ }^{13} \mathrm{C} \mathrm{NMR}\left(\mathrm{CDCl}_{3}\right.$, 75MHz): $\delta 7.5,9.9,29.7,35.1,51.4,71.9,123.5,126.8,147.2,149.1,216.1 ; \mathrm{HRMS}$ for $\mathrm{C}_{12} \mathrm{H}_{16} \mathrm{NO}_{4}$ $(\mathrm{M}+1)^{+}$, calcd. 238.1074, found 238.1075.

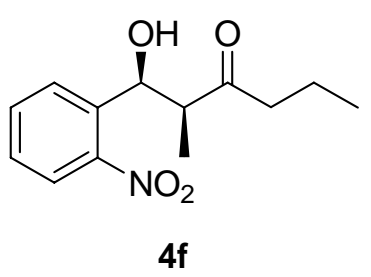

Yield: 75\%; For syn isomer: enantiomeric excess : 96\%; $[\alpha]^{20}{ }_{\mathrm{D}}=-23.6(\mathrm{c}=$ $\left.0.5, \mathrm{CHCl}_{3}\right) ;{ }^{1} \mathrm{H} \mathrm{NMR}\left(300 \mathrm{MHz}, \mathrm{CDCl}_{3}\right): \delta 0.88-0.94(3 \mathrm{H}, \mathrm{t}, J=7.4 \mathrm{~Hz})$, 1.01-1.04 (3H, d, $J=7.4 \mathrm{~Hz}), 1.58-1.65(2 \mathrm{H}, \mathrm{m}), 2.44-2.54(2 \mathrm{H}, \mathrm{m})$, 2.81-2.83 (1H, m), $3.57(1 \mathrm{H}, \mathrm{d}, J=2.3 \mathrm{~Hz}), 5.23(1 \mathrm{H}, \mathrm{t}, J=2.6 \mathrm{~Hz}), 7.51$ $(2 \mathrm{H}, \mathrm{d}, J=8.9 \mathrm{~Hz}), 8.20(2 \mathrm{H}, \mathrm{d}, J=8.9 \mathrm{~Hz}) ;{ }^{13} \mathrm{C} \mathrm{NMR}\left(\mathrm{CDCl}_{3}, 75 \mathrm{MHz}\right): \delta 9.7,13.7,16.9,43.8$, 51.5, 71.9, 123.5, 126.8, 134.8, 149.1, 215.7; HRMS for $\mathrm{C}_{13} \mathrm{H}_{18} \mathrm{NO}_{4}(\mathrm{M}+1)^{+}$, calcd. 252.1230, found 252.1231 .

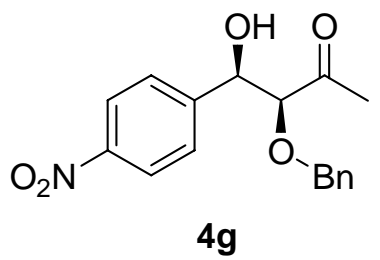

Yield: 98\%; For syn isomer: enantiomeric excess : 97\%; $[\alpha]^{20}{ }_{\mathrm{D}}=-72.4$ $\left(\mathrm{c}=0.25, \mathrm{CHCl}_{3}\right) ;{ }^{1} \mathrm{H} \mathrm{NMR}\left(300 \mathrm{MHz}, \mathrm{CDCl}_{3}\right): \delta 2.21(3 \mathrm{H}, \mathrm{s}), 3.13-3.15$ (1H, br), $3.96(1 \mathrm{H}, \mathrm{d}, J=3.6 \mathrm{~Hz}), 4.35(1 \mathrm{H}, \mathrm{d}, J=11.5 \mathrm{~Hz}), 4.58(1 \mathrm{H}, \mathrm{d}, J$ $=11.5 \mathrm{~Hz}), 5.06-5.10(1 \mathrm{H}, \mathrm{m}), 7.03-7.10(2 \mathrm{H}, \mathrm{m}), 7.22-7.30(3 \mathrm{H}, \mathrm{m}), 7.49$ $(2 \mathrm{H}, \mathrm{d}, J=8.9 \mathrm{~Hz}), 8.17(2 \mathrm{H}, \mathrm{d}, J=8.9 \mathrm{~Hz}) ;{ }^{13} \mathrm{C} \mathrm{NMR}\left(\mathrm{CDCl}_{3}, 75 \mathrm{MHz}\right): \delta 27.8,73.5,73.8,87.0$, 123.5, 127.1, 128.2, 128.5, 128.6, 136.0, 147.5, 147.6, 209.8; HRMS for $\mathrm{C}_{17} \mathrm{H}_{18} \mathrm{NO}_{5}(\mathrm{M}+1)^{+}$, calcd. 316.1174, found 316.1181 . 
<smiles>CC(=O)C(OCc1ccccc1)C(O)c1ccc(Cl)cc1</smiles>

4h

Yield: 91\%; For syn isomer: enantiomeric excess : 92\%; $[\alpha]^{20}{ }_{\mathrm{D}}=-59.8$ $\left(\mathrm{c}=1.0, \mathrm{CHCl}_{3}\right) ;{ }^{1} \mathrm{H} \mathrm{NMR}\left(300 \mathrm{MHz}, \mathrm{CDCl}_{3}\right): \delta 2.12(3 \mathrm{H}, \mathrm{s}), 3.03(1 \mathrm{H}, \mathrm{br})$, $3.90(1 \mathrm{H}, \mathrm{d}, J=4.1 \mathrm{~Hz}), 4.32-4.59(2 \mathrm{H}, \mathrm{AB}, J=27 \mathrm{~Hz}, 11.6 \mathrm{~Hz}), 4.91(1 \mathrm{H}$, $\mathrm{d}, J=4.0 \mathrm{~Hz}), 7.09-7.12(2 \mathrm{H}, \mathrm{m}), 7.21-7.31(7 \mathrm{H}, \mathrm{m}) ;{ }^{13} \mathrm{C} \mathrm{NMR}\left(\mathrm{CDCl}_{3}\right.$, 75MHz): $\delta 27.8,73.6,73.7,87.8,127.6,128.1,128.3,128.5,133.8,136.3,138.3,209.8$; HRMS for $\mathrm{C}_{17} \mathrm{H}_{18} \mathrm{ClO}_{3}(\mathrm{M}+1)^{+}$, calcd. 305.0939, found 305.0940.<smiles>CC(=O)[C@H](OBr)[C@@H](O)c1cccc2ccccc12</smiles>

$4 \mathbf{i}$

Yield: 99\%; For syn isomer: enantiomeric excess : $>99 \%$; $[\alpha]^{20}{ }_{\mathrm{D}}=-95.5(\mathrm{c}=$ 1.0, $\left.\mathrm{CHCl}_{3}\right) ;{ }^{1} \mathrm{H} \mathrm{NMR}\left(300 \mathrm{MHz}, \mathrm{CDCl}_{3}\right): \delta 2.21(3 \mathrm{H}, \mathrm{s}), 3.05(1 \mathrm{H}, \mathrm{br})$, 4.01-4.39 (3H, m), $5.80(1 \mathrm{H}, \mathrm{s}), 6.90-7.06(2 \mathrm{H}, \mathrm{m}), 7.13-7.30(5 \mathrm{H}, \mathrm{m}), 7.47-7.52$ $(3 \mathrm{H}, \mathrm{m}), 7.69(1 \mathrm{H}, \mathrm{d}, J=7.0 \mathrm{~Hz}), 7.80-7.88(2 \mathrm{H}, \mathrm{m}), 7.96-8.01(2 \mathrm{H}, \mathrm{m}) ;{ }^{13} \mathrm{C}$ $\mathrm{NMR}\left(\mathrm{CDCl}_{3}, 75 \mathrm{MHz}\right): \delta 27.9,71.7,74.0,86.5,122.4,124.1,125.2,125.6$, 126.4, 128.0, 128.3, 128.5, 129.0, 129.8, 133.7, 135.6, 136.3, 210.5; HRMS for $\mathrm{C}_{21} \mathrm{H}_{21} \mathrm{NO}_{3}(\mathrm{M}+1)^{+}$, calcd. 321.1485, found 321.1487.<smiles>CC(=O)C(OCc1ccccc1)C(O)c1ccccc1</smiles>

4j

Yield: 76\%; For syn isomer: enantiomeric excess :86\%; $[\alpha]^{20}{ }_{\mathrm{D}}=-68.0(\mathrm{c}=1.0$, $\left.\mathrm{CHCl}_{3}\right) ;{ }^{1} \mathrm{H}$ NMR $\left(300 \mathrm{MHz}, \mathrm{CDCl}_{3}\right): \delta 2.08(3 \mathrm{H}, \mathrm{s}), 2.9(1 \mathrm{H}, \mathrm{br}), 3.94(1 \mathrm{H}, \mathrm{d}, J$ $=4.3 \mathrm{~Hz}), 4.30-4.59(2 \mathrm{H}, \mathrm{AB}, J=46.7 \mathrm{~Hz}, 11.7 \mathrm{~Hz}), 4.94(1 \mathrm{H}, \mathrm{d}, J=4.0 \mathrm{~Hz})$, 7.15-7.17 (2H, m), 7.28-7.35 (8H, m); ${ }^{13} \mathrm{C} \mathrm{NMR}\left(\mathrm{CDCl}_{3}, 75 \mathrm{MHz}\right): \delta 27.8,73.5$, 74.4, 88.2, 126.3, 128.1, 128.2, 128.4, 128.5, 136.6, 139.7, 209.8; HRMS for $\mathrm{C}_{17} \mathrm{H}_{19} \mathrm{O}_{3}(\mathrm{M}+1)^{+}$, calcd. 271.1329, found 271.1332<smiles>CCCC(=O)CC(O)c1ccc([N+](=O)[O-])cc1</smiles>

4k

Yield: 92\%; Enantiomeric excess : 88\%; $[\alpha]^{20}{ }_{\mathrm{D}}=-53.4(\mathrm{c}=0.5$, $\left.\mathrm{CHCl}_{3}\right) ;{ }^{1} \mathrm{H} \mathrm{NMR}\left(300 \mathrm{MHz}, \mathrm{CDCl}_{3}\right): \delta 0.91(3 \mathrm{H}, \mathrm{t}, J=7.5 \mathrm{~Hz})$, 1.57-1.69 (2H, m), $2.42(2 \mathrm{H}, \mathrm{t}, J=7.4 \mathrm{~Hz}), 2.79-2.86(2 \mathrm{H}, \mathrm{m}), 3.70$ $(1 \mathrm{H}, \mathrm{s}), 5.23-5.28(1 \mathrm{H}, \mathrm{m}), 7.53(2 \mathrm{H}, \mathrm{d}, J=8.7 \mathrm{hz}), 8.18(2 \mathrm{H}, \mathrm{m}) ;{ }^{13} \mathrm{C}$ $\mathrm{NMR}\left(\mathrm{CDCl}_{3}, 75 \mathrm{MHz}\right): \delta 13.6,17.0,45.5,50.6,69.0,123.7,126.4,147.3,150.2,211.0$; HRMS for $\mathrm{C}_{12} \mathrm{H}_{16} \mathrm{NO}_{4}(\mathrm{M}+1)^{+}$, calcd. 238.1074, found 238.1072. 
<smiles>CC(C)CC(=O)C[C@@H](O)c1ccc([N+](=O)[O-])cc1</smiles>

41

Yield: 56\%; Enantiomeric excess : 85\%; $[\alpha]^{20}{ }_{D}=-42.5(\mathrm{c}=1.0$, $\left.\mathrm{CHCl}_{3}\right) ;{ }^{1} \mathrm{H} \mathrm{NMR}\left(300 \mathrm{MHz}, \mathrm{CDCl}_{3}\right): \delta 0.83-0.91(6 \mathrm{H}, \mathrm{d}, J=6.8 \mathrm{~Hz})$, 2.08-2.15 (1H, m), $2.30(2 \mathrm{H}, \mathrm{d}, J=7.0 \mathrm{~Hz}), 2.77-2.79(2 \mathrm{H}, \mathrm{m}), 3.75$ $(1 \mathrm{H}, \mathrm{d}, J=3.2 \mathrm{~Hz}), 5.24-5.27(1 \mathrm{H}, \mathrm{m}), 7.52(2 \mathrm{H}, \mathrm{d}, J=8.5 \mathrm{~Hz}), 8.18$ $(2 \mathrm{H}, \mathrm{d}, J=8.7 \mathrm{~Hz}) ;{ }^{13} \mathrm{C} \mathrm{NMR}\left(\mathrm{CDCl}_{3}, 75 \mathrm{MHz}\right): \delta 22.5,24.6,51.1,52.5,68.9,123.7,126.4,147.3$, 150.2, 210.7; HRMS for $\mathrm{C}_{13} \mathrm{H}_{18} \mathrm{NO}_{4}(\mathrm{M}+1)^{+}$, calcd. 252.1225, found 252.1227 .<smiles>O=C1CCCCC1C(O)c1ccc([N+](=O)[O-])cc1</smiles>
$4 \mathrm{~m}$

Yield: 99\%; For anti isomer: enantiomeric excess: 98\%; $[\alpha]_{\mathrm{D}}^{20}=-9.6$ $\left(\mathrm{c}=1.0, \mathrm{CHCl}_{3}\right) ;{ }^{1} \mathrm{H}$ NMR $\left(300 \mathrm{MHz}, \mathrm{CDCl}_{3}\right): \delta$ 1.32-1.46 $(1 \mathrm{H}, \mathrm{m})$, 1.46-1.76 (2H, m), 1.76-1.91 (1H, m), 2.04-2.19 (1H, m), 2.28-2.44 (1H, m), 2.44-2.72 (2H, m), $4.06(1 \mathrm{H}, \mathrm{d}, J=3.0 \mathrm{~Hz}), 4.81-4.91(1 \mathrm{H}, \mathrm{dd}, J=3.0$

$\mathrm{Hz}, 3.2 \mathrm{~Hz}, 8.3 \mathrm{~Hz}), 7.43-7.67$ (2H, m), 8.10-8.37 (2H, m); ${ }^{13} \mathrm{C} \mathrm{NMR}\left(\mathrm{CDCl}_{3}, 75 \mathrm{MHz}\right): \delta 24.7$, $27.6,30.7,42.6,57.1,74.0,123.5,127.8,147.6,148.3,214.6$.

\section{References:}

1. Mithcell, J. M.; Finney, N. S. Tetrahedron Lett. 2000, 41, 8431-8434.

2. (a) Samanta, S.; Liu, J.; Dodda, R.; Zhao, C.-G. Org. Lett. 2005, 7, 5321. (b) Chen, J.-R.; Lu, H.-H.; Li, X.-Y.; Chen, L.; Wan, J.; Xiao, W.-J. Org. Lett. 2005, 7, 4543. (c) Tang, Z.; Yang, Z.-H.; Chen, X.-H.; Cun, L.-F.; Mi, A.-Q.; Jiang, Y.-Z.; Gong, L.-Z. J. Am. Chem. Soc. 2005, 127, 9285. (d) Kano, T.; Tokuda, O.; Takai, J.; Maruoka, K. Chem.-Asian. J. 2006, 210-215.

3. Jung, C.-K.; Garner, S. A.; Krische, M. J. Org. Lett. 2006, 8, 519-522.

4. Nakadai, M.; Saito, S.; Yamamoto, H. Tetrahedron 2002, 58, 8167-8177.

5. Ramasastry, S. S. V.; Zhang, H.; Tanaka, F.; Barbas, C. F., III. J. Am. Chem. Soc. 2007, 129, 288-289. 


\section{HPLC conditions \& ${ }^{1}$ HNMR determination of diastereoselectivity:}

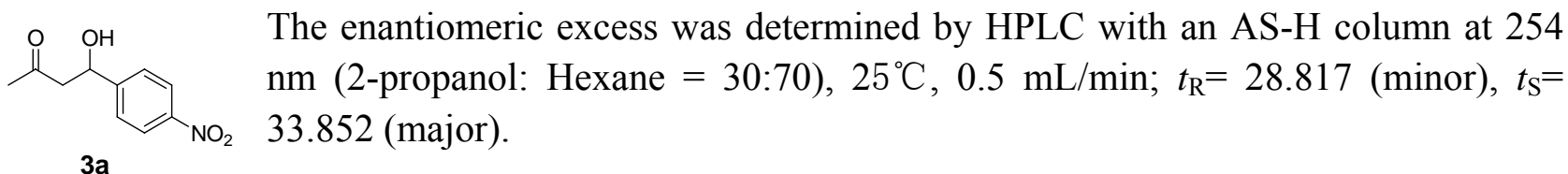
The enantiomeric excess was determined by HPLC with an OJ-H column at $254 \mathrm{~nm}$ (2-propanol: Hexane $=20: 80), 25^{\circ} \mathrm{C}, 0.8 \mathrm{~mL} / \mathrm{min} ; t_{\mathrm{R}}=22.355$ (minor), $t_{\mathrm{S}}=24.083$ 3b $\mathrm{NO}_{2}$ (major).

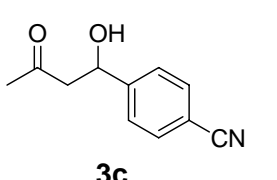

$3 c$<smiles>CC(=O)CC(O)c1cccc2ccccc12</smiles>

3d<smiles>CC(=O)CC(O)c1ccccc1Cl</smiles>

3e<smiles>CC(=O)CC(O)c1cccc(Br)c1</smiles>

$3 f$

The enantiomeric excess was determined by HPLC with an AS-H column at 254 $\mathrm{nm}$ (2-propanol: Hexane $=10: 90), 25^{\circ} \mathrm{C}, 0.8 \mathrm{~mL} / \mathrm{min} ; t_{\mathrm{R}}=16.956$ (minor), $t_{\mathrm{S}}=$ 17.488 (major).

The enantiomeric excess was determined by HPLC with an AS-H column at $254 \mathrm{~nm}$ (2-propanol: Hexane $=10: 90$ ), $25^{\circ} \mathrm{C}, 0.6 \mathrm{~mL} / \mathrm{min} ; t_{\mathrm{S}}=24.087$ (major) $t_{\mathrm{R}}=34.623$ (minor),.

The enantiomeric excess was determined by HPLC with an AS-H column at 254 $\mathrm{nm}$ (2-propanol: Hexane $=30: 70), 25^{\circ} \mathrm{C}, 0.5 \mathrm{~mL} / \mathrm{min} ; t_{\mathrm{R}}=25.689$ (minor), $t_{\mathrm{S}}=$ 43.574 (major).

The enantiomeric excess was determined by HPLC with an AS-H column at $254 \mathrm{~nm}$ (2-propanol: Hexane $=15: 85$ ), $25^{\circ} \mathrm{C}, 0.8 \mathrm{~mL} / \mathrm{min} ; t_{\mathrm{S}}=12.105$ (major), $t_{\mathrm{R}}=13.192$ (minor).

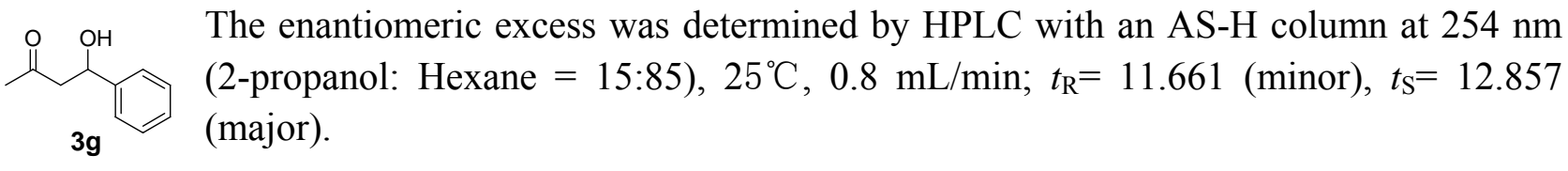

The enantiomeric excess was determined by HPLC with an AS-H column at 254 $\mathrm{nm}$ (2-propanol: Hexane $=10: 90), 25^{\circ} \mathrm{C}, 0.8 \mathrm{~mL} / \mathrm{min} ; t_{\mathrm{R}}=37.701$ (minor), $t_{\mathrm{S}}=$ 41.043 (major).

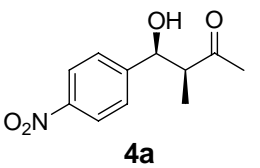

The enantiomeric excess was determined by HPLC with an AS-H column at 254 $\mathrm{nm}$ (2-propanol: Hexane $=30: 70), 25^{\circ} \mathrm{C}, 0.5 \mathrm{~mL} / \mathrm{min}$; for syn isomer: $t_{\mathrm{R}}=19.009$ 
(minor), $t_{\mathrm{S}}=21.750$ (major); $\mathrm{HOC} \underline{\mathrm{H}}(\mathrm{Ar}): \delta 5.28$ (syn, d, $J=2.8 \mathrm{~Hz}$ ), 4.87 (anti, d, $J=7.9 \mathrm{~Hz}$ ).

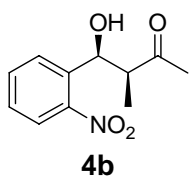

The enantiomeric excess was determined by HPLC with an AS-H column at $254 \mathrm{~nm}$ (2-propanol: Hexane $=30: 70), 25^{\circ} \mathrm{C}, 0.6 \mathrm{~mL} / \mathrm{min}$; for syn isomer: $t_{\mathrm{R}}=14.598$ (minor), $t_{\mathrm{S}}=15.097$ (major); $\mathrm{HOC} \underline{\mathrm{H}}(\mathrm{Ar}): \delta 5.78$ (syn, d, $J=1.9 \mathrm{~Hz}$ ), 5.67 (anti, dd, $J=9.4 \mathrm{~Hz}$, $1.9 \mathrm{~Hz})$.

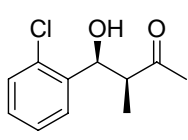

The enantiomeric excess was determined by HPLC with an AS-H column at $254 \mathrm{~nm}$ (2-propanol: Hexane $=10: 90), 25^{\circ} \mathrm{C}, 0.6 \mathrm{~mL} / \mathrm{min}$; for syn isomer: $t_{\mathrm{R}}=17.379$ (minor), $t_{\mathrm{S}}=20.310$ (major); HOC $\underline{H}(\mathrm{Ar}): \delta 5.48$ (syn, d, $J=1.7 \mathrm{~Hz}$ ), 5.45 (anti, d, $J=2.3 \mathrm{~Hz}$ )

$4 c$<smiles>CC(=O)C(C)C(O)c1cccc2ccccc12</smiles>

The enantiomeric excess was determined by HPLC with an AS-H column at $254 \mathrm{~nm}$ (2-propanol: Hexane $=20: 80), 25^{\circ} \mathrm{C}, 0.8 \mathrm{~mL} / \mathrm{min}$; for syn isomer: $t_{\mathrm{R}}=11.41$ (minor), $t_{\mathrm{S}}=12.15$ (major); HOC $\underline{\mathrm{H}}(\mathrm{Ar}): \delta 5.94$ (syn, d, $\left.J=3.0 \mathrm{~Hz}\right), 5.49$ (anti, d, $J=8.1 \mathrm{~Hz}$ ).

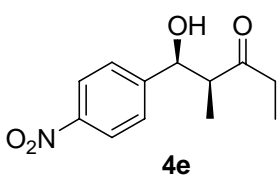

The enantiomeric excess was determined by HPLC with an AS-H column at 254 nm (2-propanol: Hexane $=5: 95), 20^{\circ} \mathrm{C}, 0.8 \mathrm{~mL} / \mathrm{min}$; for syn isomer: $t_{\mathrm{S}}=40.549$ (major) $t_{\mathrm{R}}=43.244$ (minor); $\mathrm{HOC} \underline{\mathrm{H}}(\mathrm{Ar}): \delta 5.23$ (syn, t, $J=2.6 \mathrm{~Hz}$ ), 4.88 (anti, dd, $J$ $=7.5 \mathrm{~Hz}, 5.5 \mathrm{~Hz})^{4}$.

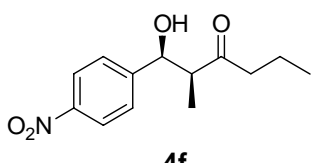

The enantiomeric excess was determined by HPLC with an AS-H column at 254 $\mathrm{nm}$ (2-propanol: Hexane $=5: 95), 25^{\circ} \mathrm{C}, 0.8 \mathrm{~mL} / \mathrm{min}$; for syn isomer: $t_{\mathrm{R}}=38.606$ (minor), $t_{\mathrm{S}}=43.452$ (major); $\mathrm{HOC} \underline{\mathrm{H}}(\mathrm{Ar}$ ): $\delta 5.23$ (syn, br s), 4.88 (anti, dd, $J=$ $7.2 \mathrm{~Hz}, 4.9 \mathrm{~Hz})$.

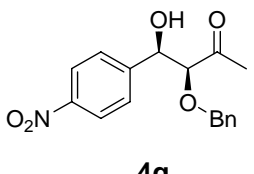

The enantiomeric excess was determined by HPLC with an AS-H column at $254 \mathrm{~nm}$ (2-propanol: Hexane $=30: 70), 25^{\circ} \mathrm{C}, 0.5 \mathrm{~mL} / \mathrm{min}$; for syn isomer: $t_{\mathrm{R}}=17.258$ (minor), $t_{\mathrm{S}}=27.337$ (major); HOC $\underline{\mathrm{H}}(\mathrm{Ar}): \delta 5.09$ (syn, d, $J=3.6 \mathrm{~Hz}$ ), 5.04 (anti, d, $J=6.4 \mathrm{~Hz}$ ).

49<smiles>CC(=O)C(O)C(O)c1ccc(Cl)cc1</smiles>

$4 \mathrm{~h}$

The enantiomeric excess was determined by HPLC with an AS-H column at 254 $\mathrm{nm}$ (2-propanol: Hexane $=20: 80), 25^{\circ} \mathrm{C}, 0.8 \mathrm{~mL} / \mathrm{min}$; for syn isomer: $t_{\mathrm{R}}=9.05$ (minor), $t_{\mathrm{S}}=15.07$ (major); HOC$\underline{H}(\mathrm{Ar}): \delta 4.92$ (syn, d, $J=4.1 \mathrm{~Hz}$ ), 4.88 (anti, d, $J=$ $6.4 \mathrm{~Hz})$. 
<smiles>CC(=O)[C@H](OCc1ccccc1)[C@H](O)c1cccc2ccccc12</smiles>

The enantiomeric excess was determined by HPLC with an AS-H column at 254 $\mathrm{nm}$ (2-propanol: Hexane $=20: 80), 25^{\circ} \mathrm{C}, 0.8 \mathrm{~mL} / \mathrm{min}$; for syn isomer: $t_{\mathrm{R}}=7.51$ (minor), $t_{\mathrm{S}}=10.30$ (major); $\mathrm{HOC} \underline{\mathrm{H}}(\mathrm{Ar}): \delta 5.82$ (syn, d, $J=2.5 \mathrm{~Hz}$ ), 5.74 (anti, d, $J$ $=6.2 \mathrm{~Hz})$.<smiles>CC(=O)C(OBr)C(O)c1ccccc1</smiles>

The enantiomeric excess was determined by HPLC with an AS-H column at 254 $\mathrm{nm}$ (2-propanol: Hexane $=20: 80), 25^{\circ} \mathrm{C}, 0.8 \mathrm{~mL} / \mathrm{min}$; for syn isomer: $t_{\mathrm{R}}=9.42$ (minor), $t_{\mathrm{S}}=15.65$ (major); $\mathrm{HOC} \underline{\mathrm{H}}(\mathrm{Ar}): \delta 4.97$ (syn, d, $J=4.1 \mathrm{~Hz}$ ), 4.90 (anti, d, $J$ $=6.6 \mathrm{~Hz})$.

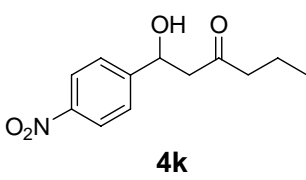

The enantiomeric excess was determined by HPLC with an AS-H column at $254 \mathrm{~nm}$ (2-propanol: Hexane $=30: 70), 25^{\circ} \mathrm{C}, 0.5 \mathrm{~mL} / \mathrm{min} ; t_{\mathrm{R}}=18.478$ (minor), $t_{\mathrm{S}}=27.158$ (major).

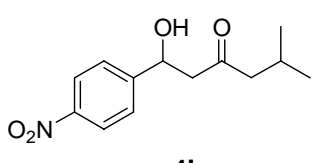

The enantiomeric excess was determined by HPLC with an AS-H column at $254 \mathrm{~nm}$ (2-propanol: Hexane $=30: 70$ ), $25^{\circ} \mathrm{C}, 0.6 \mathrm{~mL} / \mathrm{min} ; t_{\mathrm{R}}=13.727$ (minor), $t_{\mathrm{S}}=14.524$ (major).

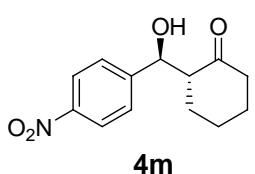

The enantiomeric excess was determined by HPLC with an AD-H column at 254 $\mathrm{nm}$ (2-propanol: Hexane $=20: 80), 25^{\circ} \mathrm{C}, 0.5 \mathrm{~mL} / \mathrm{min} ; t_{\mathrm{S}}=22.443$ (major), $t_{\mathrm{R}}=$ 28.598 (minor). HOCH$(A r): \delta 5.46$ (syn, d, J=1.9 Hz), 4.88 (anti, d, $J=8.3 \mathrm{~Hz}$ ). 
NMR spectra for catalysts 1 and 2
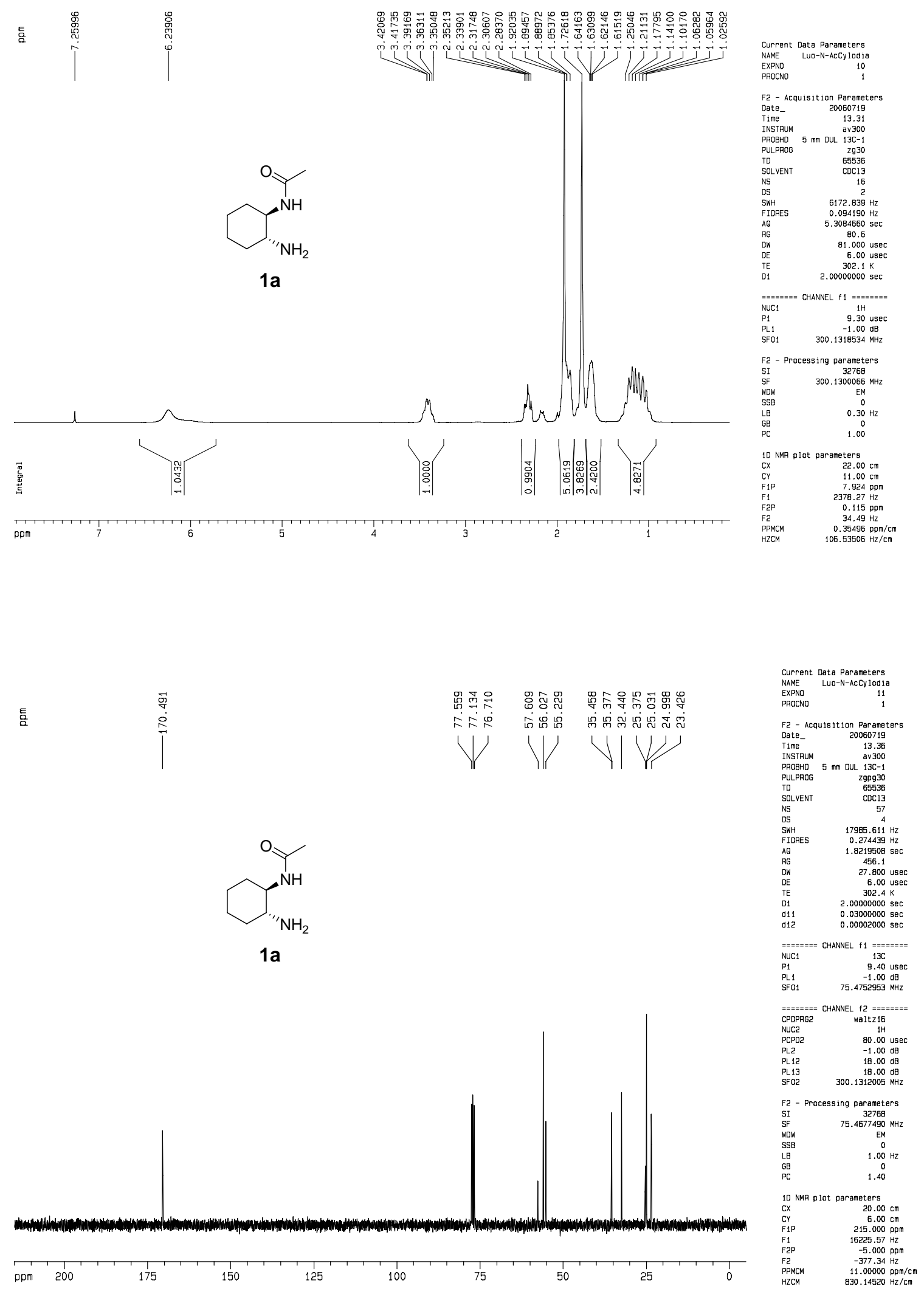

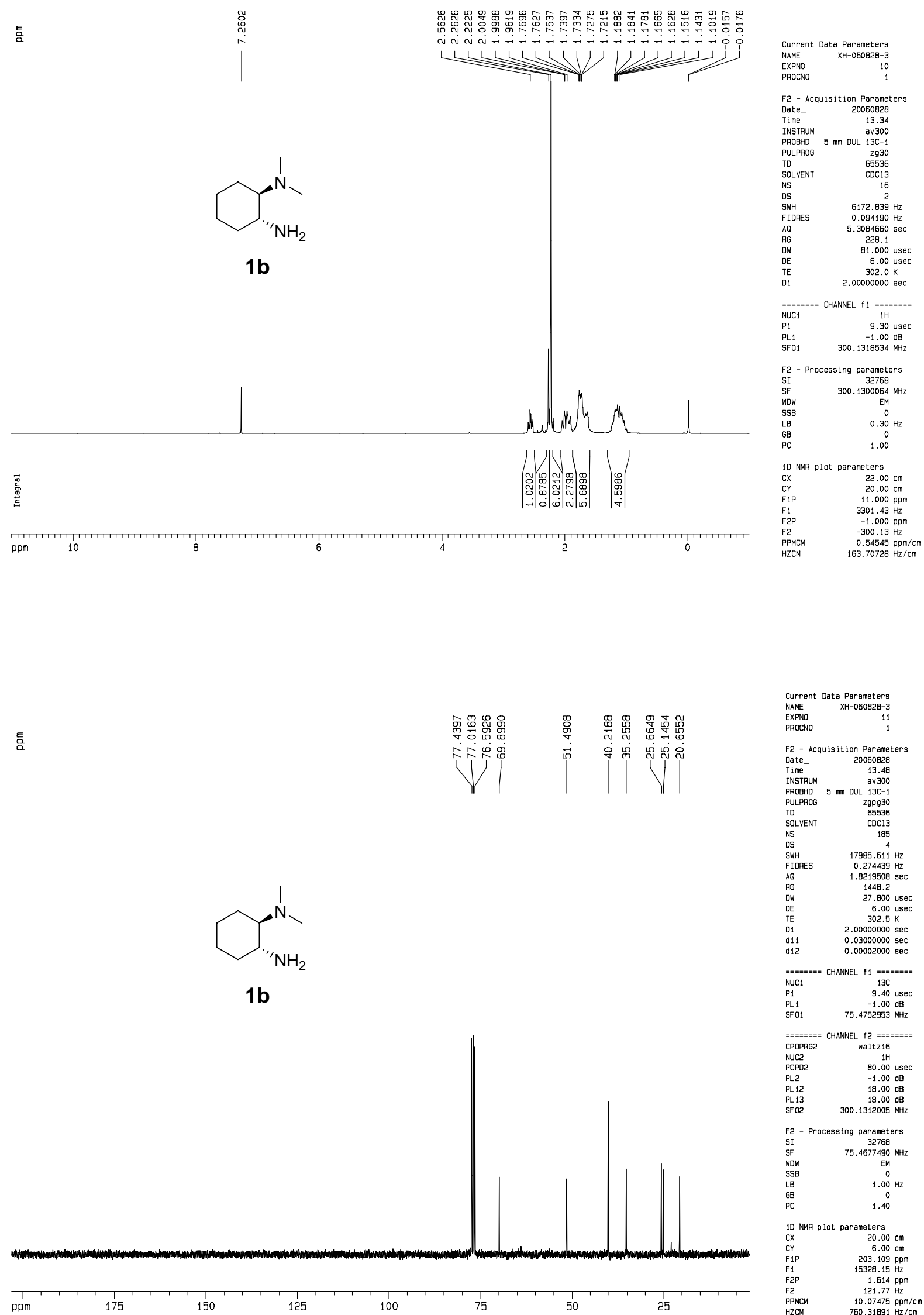


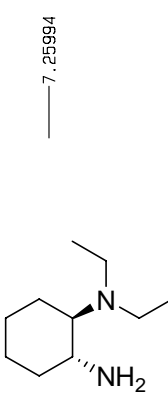

1c

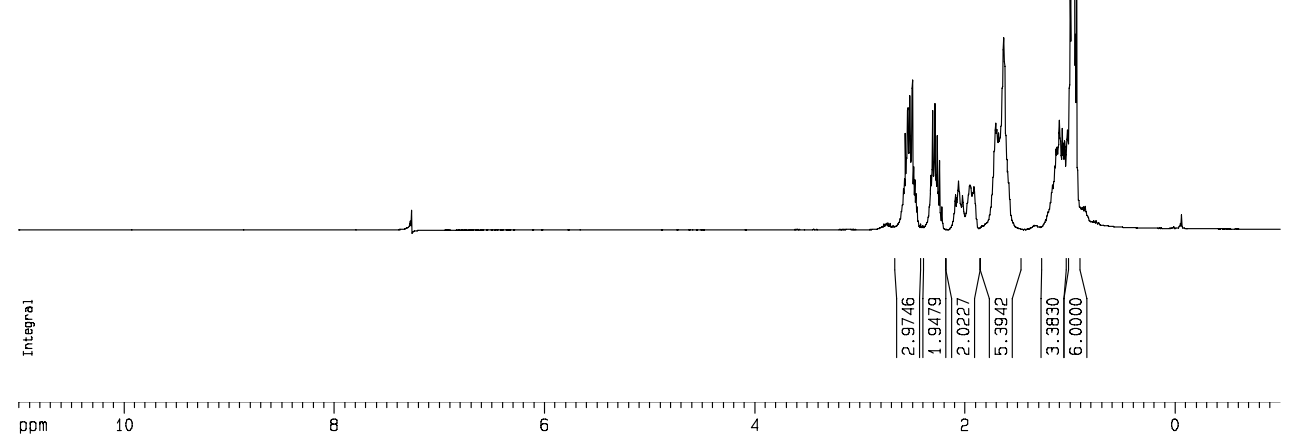

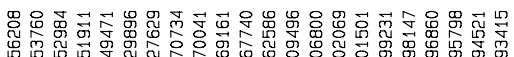

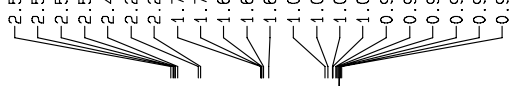

$\begin{array}{lr}\text { Current Data Parameters } \\ \text { NAME } & \text { XH-061108-2 } \\ \text { EXPNO } & 10 \\ \text { PROCNO } & 1\end{array}$

F2 - Acquisition Parameters

$\begin{array}{lr}\text { Date } & 20061109 \\ \text { Time } & 12.48\end{array}$

Instrour

PROBHO $5 \mathrm{~mm}$ DUL $13 \mathrm{C}-1$

$\begin{array}{lr}\text { PULPROG } & 2930 \\ \text { TD } & 65535 \\ \text { SOLVENT } & \text { CDC13 }\end{array}$

NS

5WH $\quad \begin{gathered}2 \\ 5172.839 \mathrm{~Hz}\end{gathered}$

AQ $\quad 5.3084660 \mathrm{sec}$

81.000 usec

$\begin{array}{lr}\text { DE } & 6.00 \text { use } \\ \text { TE } & 298.5 \mathrm{~K} \\ \text { D1 } & 2.00000000 \mathrm{se}\end{array}$

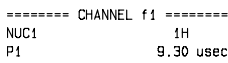

$\begin{array}{lr}\text { P1 } & \text { 9. } \\ \text { PL1 } & -1.00 \mathrm{~dB} \\ \text { SF01 } & 300.1318534 \mathrm{MHz}\end{array}$

$\begin{array}{ll}\text { F2 - Processing parameters } \\ \text { SI } & 32768 \\ \text { SF } & 300.1300064 \\ \text { SHZ }\end{array}$

$\begin{array}{lc}\text { SF } & 300.1300064 \mathrm{MHz} \\ \text { WOW } & \text { EM } \\ \text { SSB } & 0 \\ \text { LB } & 0.30 \mathrm{~Hz} \\ \text { GB } & 0 \\ \text { OB } & 1.00\end{array}$

10 NMP plot parameters

$\begin{array}{lr}\text { CX } & 22.00 \mathrm{~cm} \\ \mathrm{CY} & 11.00 \mathrm{~cm}\end{array}$

$\begin{array}{ll}F 1 P & 11.000 \mathrm{ppm} \\ F 1 & 3301.43 \mathrm{~Hz}\end{array}$

$\begin{array}{ll}\text { F1 } & 3301.43 \mathrm{~Hz} \\ \text { F2P } & -1.00 \mathrm{ppm} \\ \text { F2 } & -300.13 \mathrm{~Hz}\end{array}$

$\begin{array}{lr}\text { PPMCM } & 0.54555 \mathrm{ppm} / \mathrm{cm} \\ \text { HZCM } & 163.70728 \mathrm{~Hz} / \mathrm{cm}\end{array}$

言
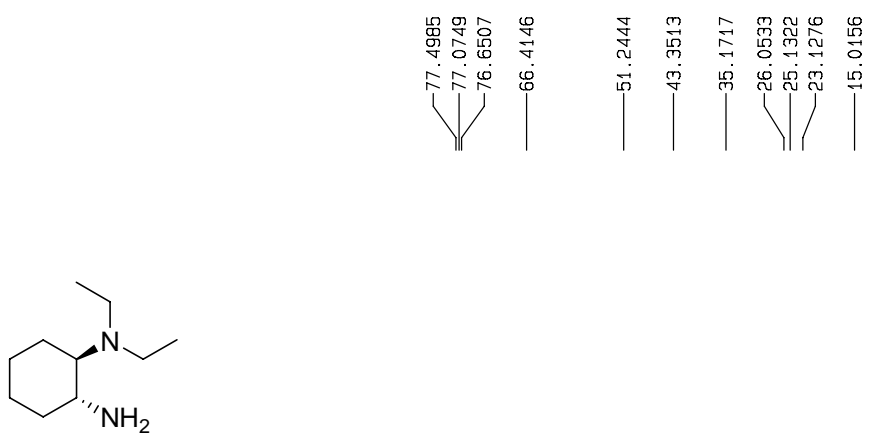

$1 c$

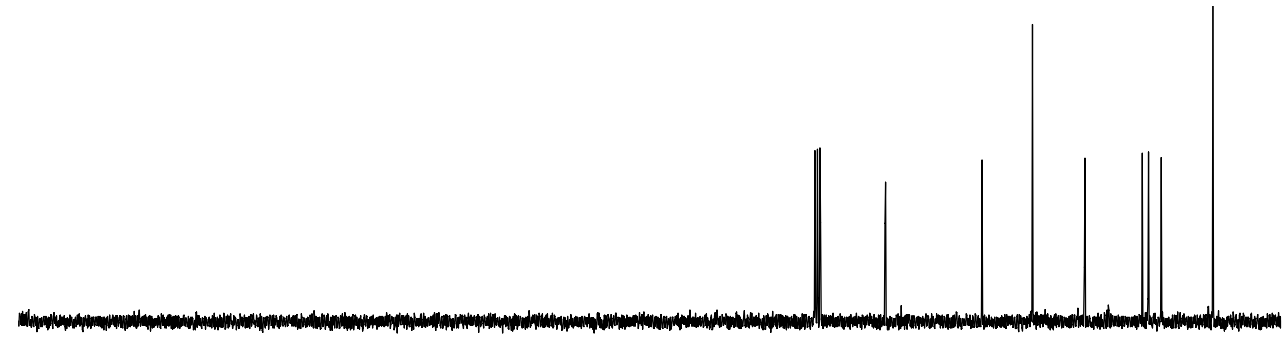

ppm

180

160

140

120

100

80

60

40

20

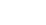



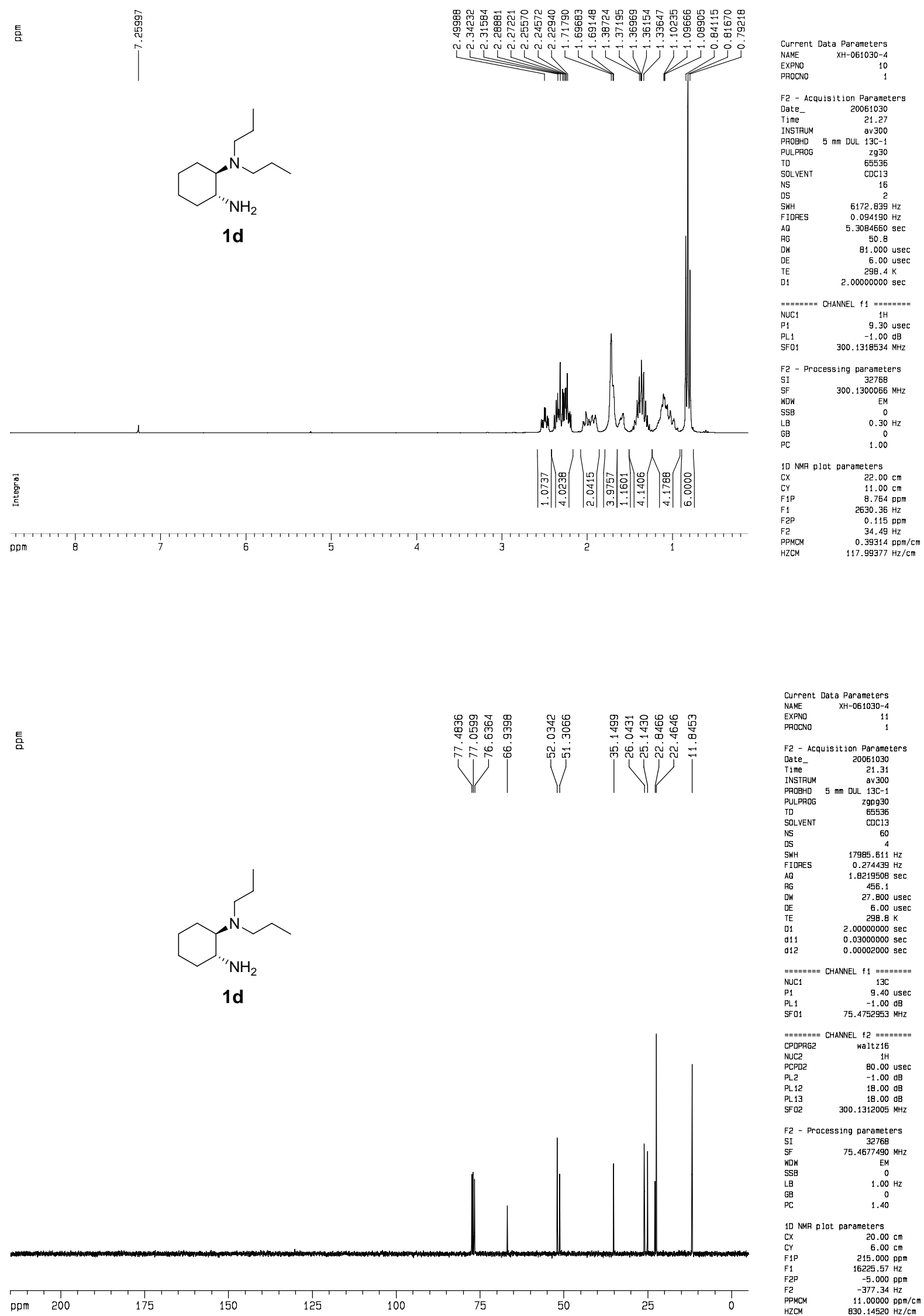

\begin{tabular}{ll}
\hline ppm & 200
\end{tabular}

150

125

100

50

$830.14520 \mathrm{~Hz} / \mathrm{cm}$ 

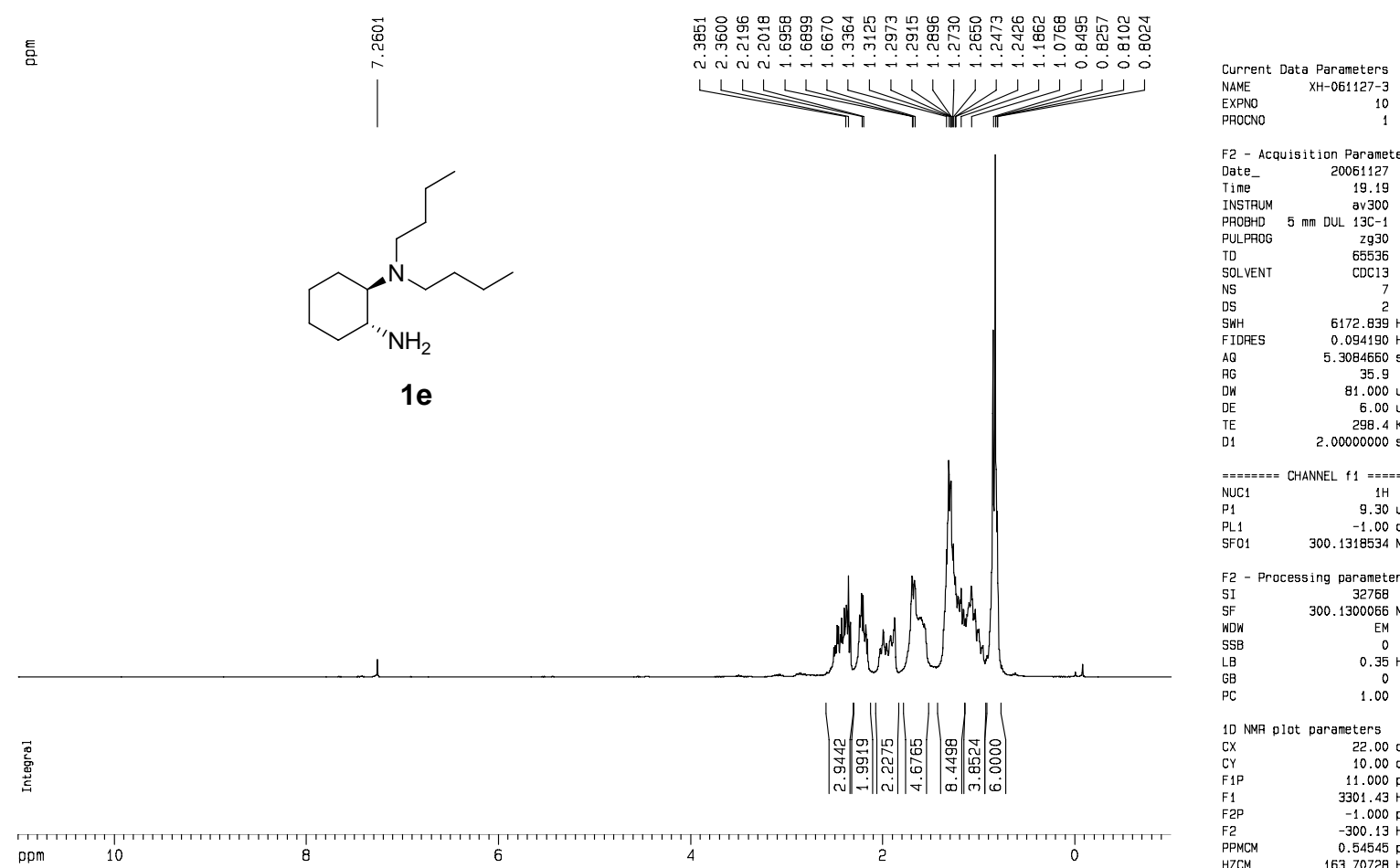

F2 - Acquisition Parameters
Date 20061127

$\begin{array}{lr}\text { Time } & 19.19 \\ \text { INSTRUM } & \text { av300 } \\ \text { PHOBHD } & 5 \mathrm{~mm} \text { DUL } 13 \mathrm{C}-1\end{array}$

PAOBHO $5 \mathrm{~mm}$ DUL $13 \mathrm{1} C-1$
PULPROG
2930

$\begin{array}{ll}\text { TD } & 65536 \\ \text { SOLVENT } & \text { CDC13 } \\ \text { NS } & \end{array}$

$\begin{array}{lr} & 2 \\ \text { SWH } & 6172.839 \mathrm{~Hz} \\ \text { FIDRES } & 0.094190 \mathrm{~Hz}\end{array}$

$\begin{array}{ll}A Q & 5.3084660 \mathrm{sec} \\ \mathrm{AG} & 35.9\end{array}$

$\begin{array}{ll}\text { OW } & 81.000 \text { usec } \\ \text { DE } & 5.00 \text { usec }\end{array}$

$== \pm== \pm=$ CHANNEL $f 1$ = $== \pm==$

$\begin{array}{lll}\text { NUC1 } & 1 \mathrm{H} \\ \mathrm{P}_{1} & 9.30 \mathrm{usec}\end{array}$

$\begin{array}{lr}\text { PL1 } & -1.00 \mathrm{~dB} \\ \text { SF01 } & 300.1318534 \mathrm{MHZ}\end{array}$

F2 - Processing parameters

$\begin{array}{lc}\text { SI } & 32768 \\ \text { SF } & 300.1300066 \mathrm{MHz} \\ \text { WDW } & \text { EM } \\ \text { SSB } & 0 \\ \text { LB } & 0.35 \mathrm{~Hz} \\ \text { GB } & 0 \\ \text { PC } & 1.00\end{array}$

10 NMP plot parameters

$\begin{array}{lr}\text { CX } & 22.00 \mathrm{~cm} \\ \mathrm{CY} & 10.00 \mathrm{~cm} \\ \mathrm{~F} & 11.000 \mathrm{~mm}\end{array}$

$\begin{array}{lr}\text { FIP } & 11.000 \mathrm{pPm} \\ \text { F1 } & 3301.43 \mathrm{~Hz} \\ \text { F2P } & -1.000 \mathrm{pDm}\end{array}$

$\begin{array}{ll}\text { F2 } & -300.13 \mathrm{~Hz} \\ \text { PPMCM } & 0.54545 \mathrm{ppm} / \mathrm{Cm}\end{array}$

10

言
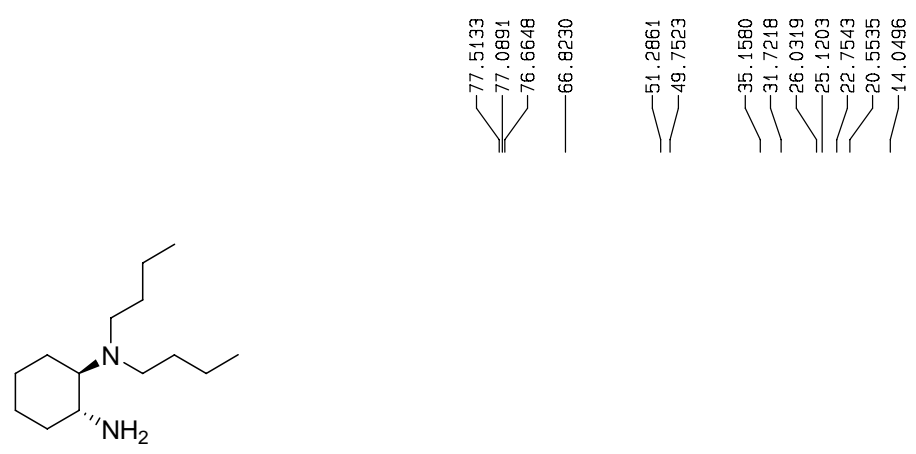

$0.54545 \mathrm{ppm} / \mathrm{cm}$
$163.70728 \mathrm{~Hz} / \mathrm{cm}$

$1 e$

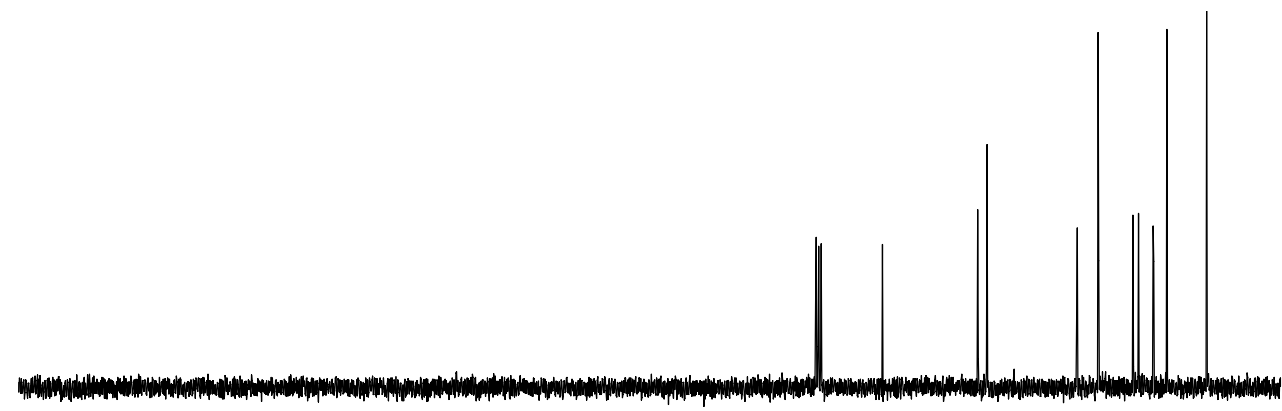



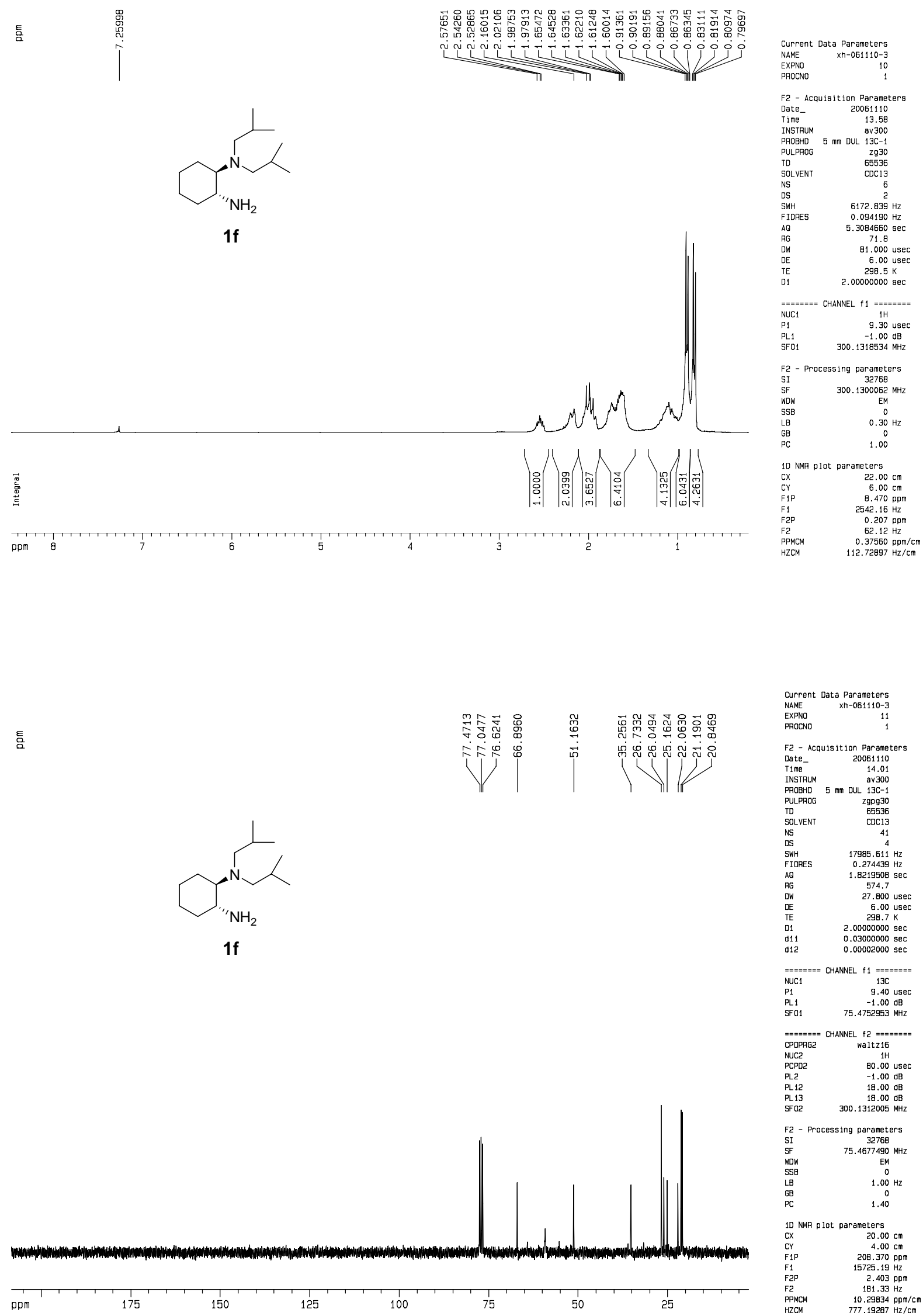

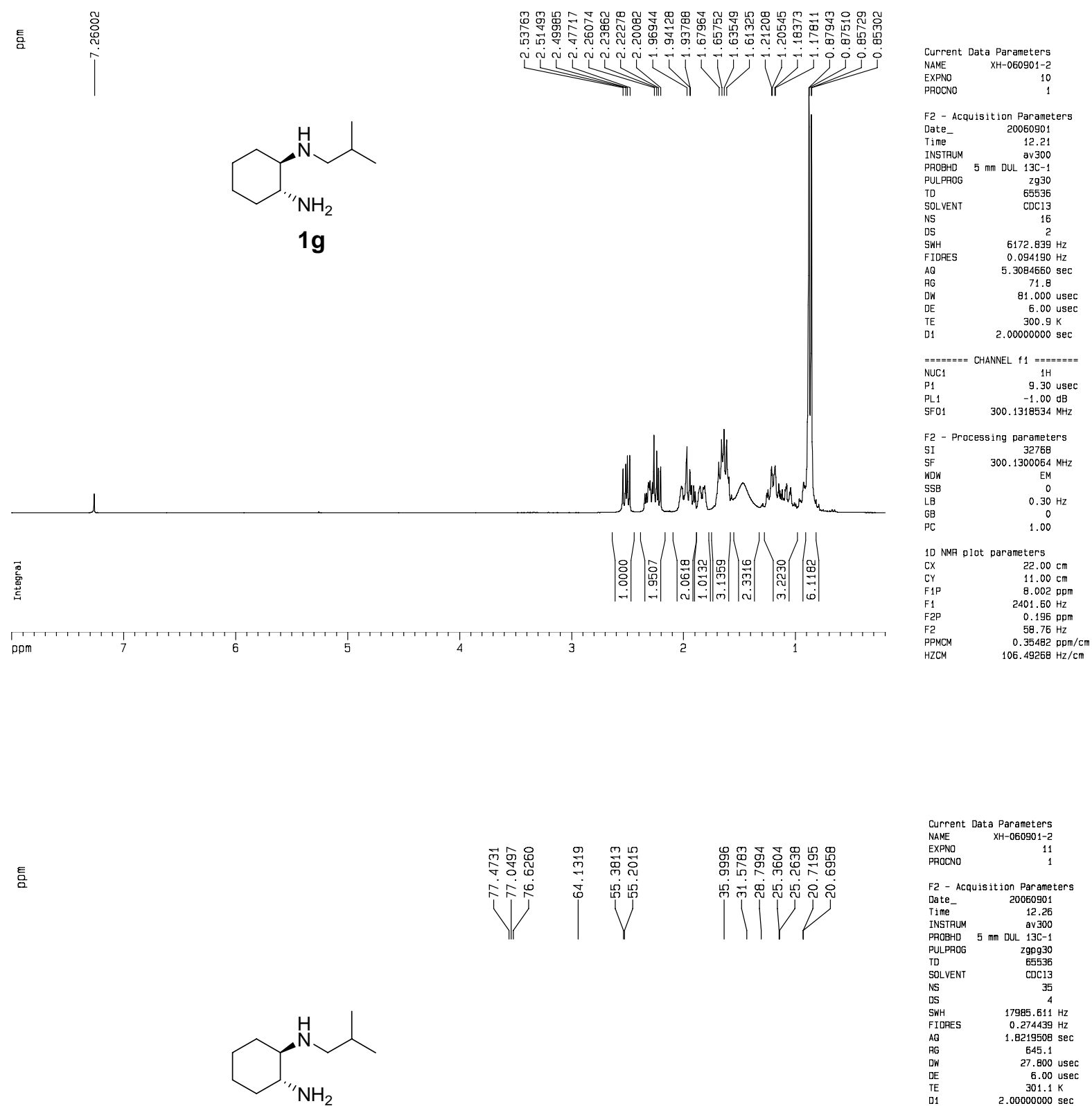

$1 \mathrm{~g}$

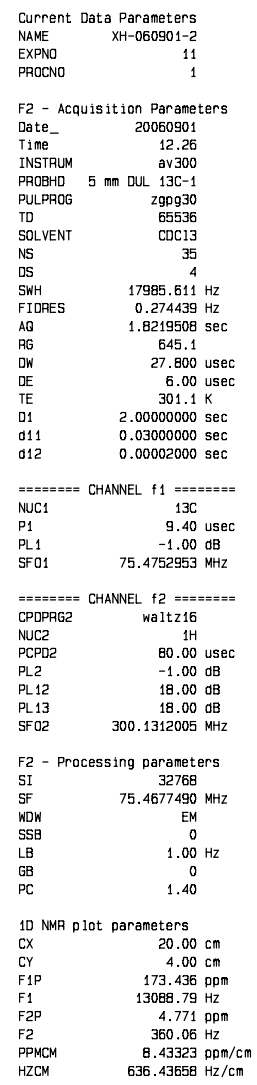



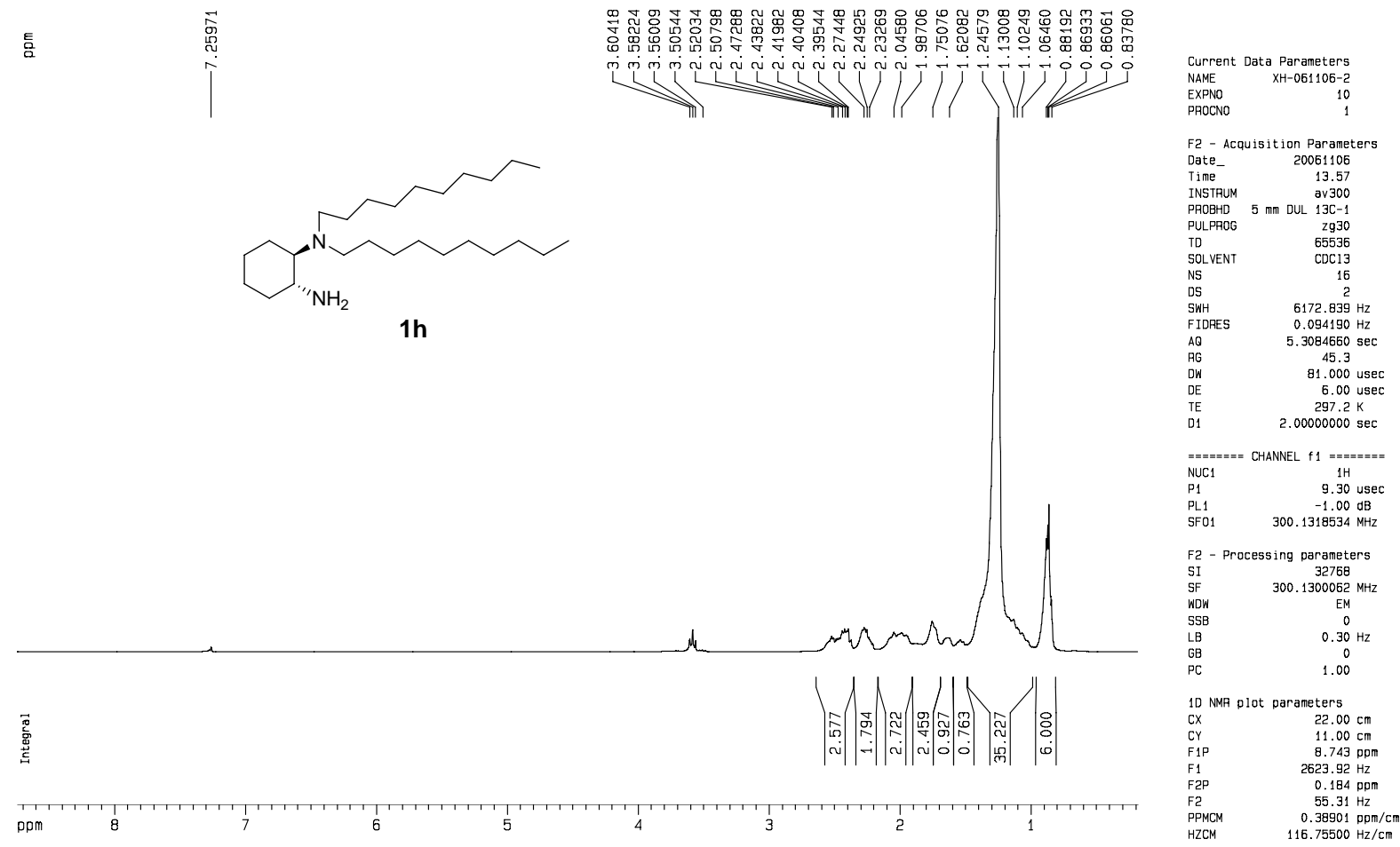

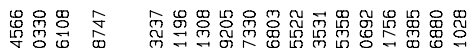

i

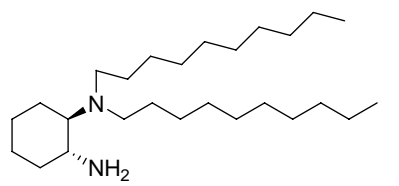

$1 h$

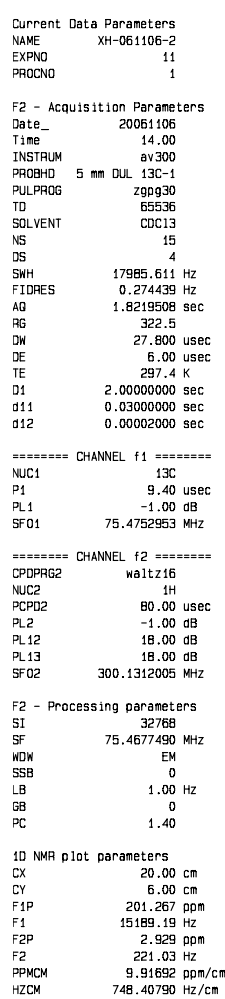



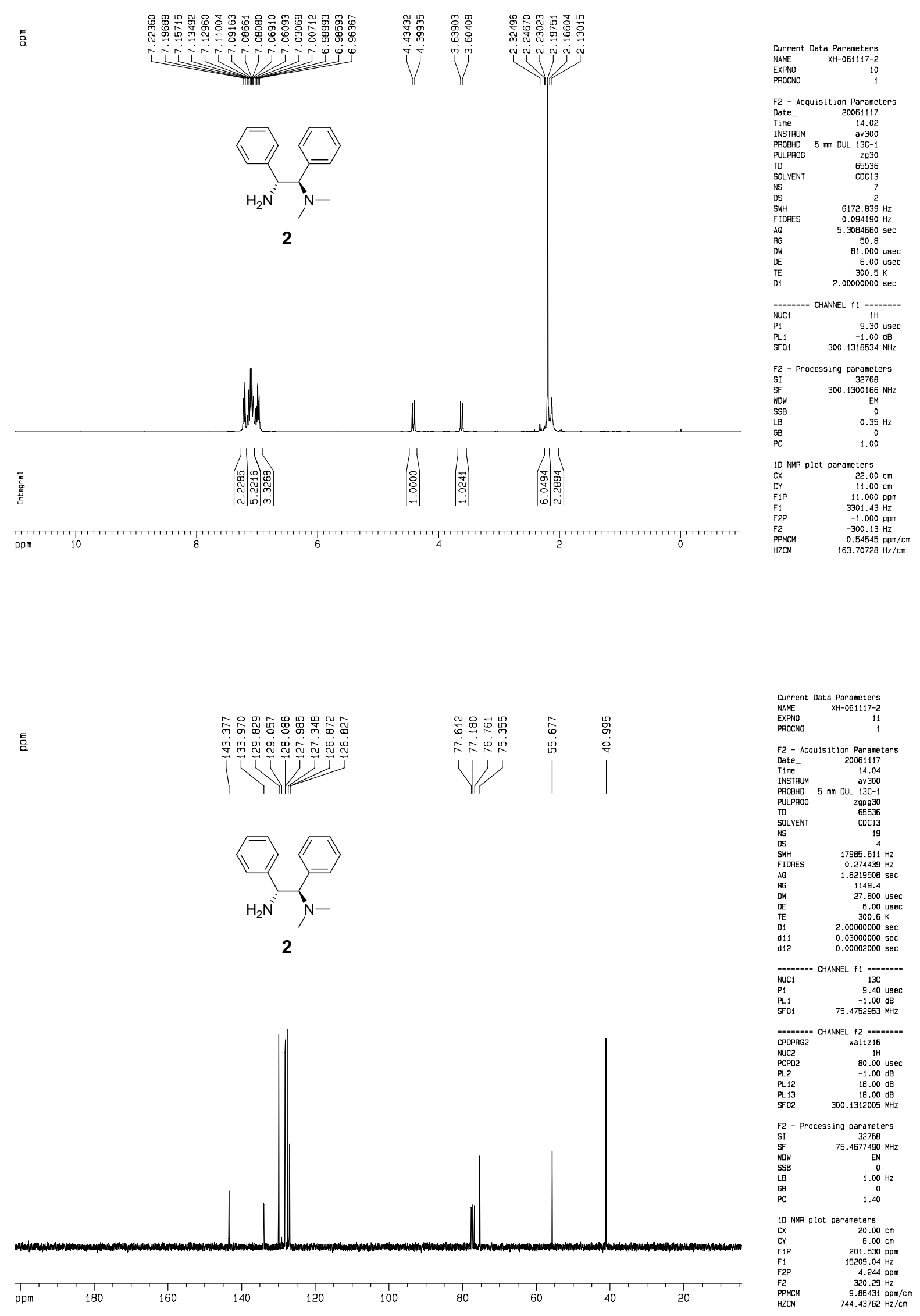
NMR spectra for all the Aldol products
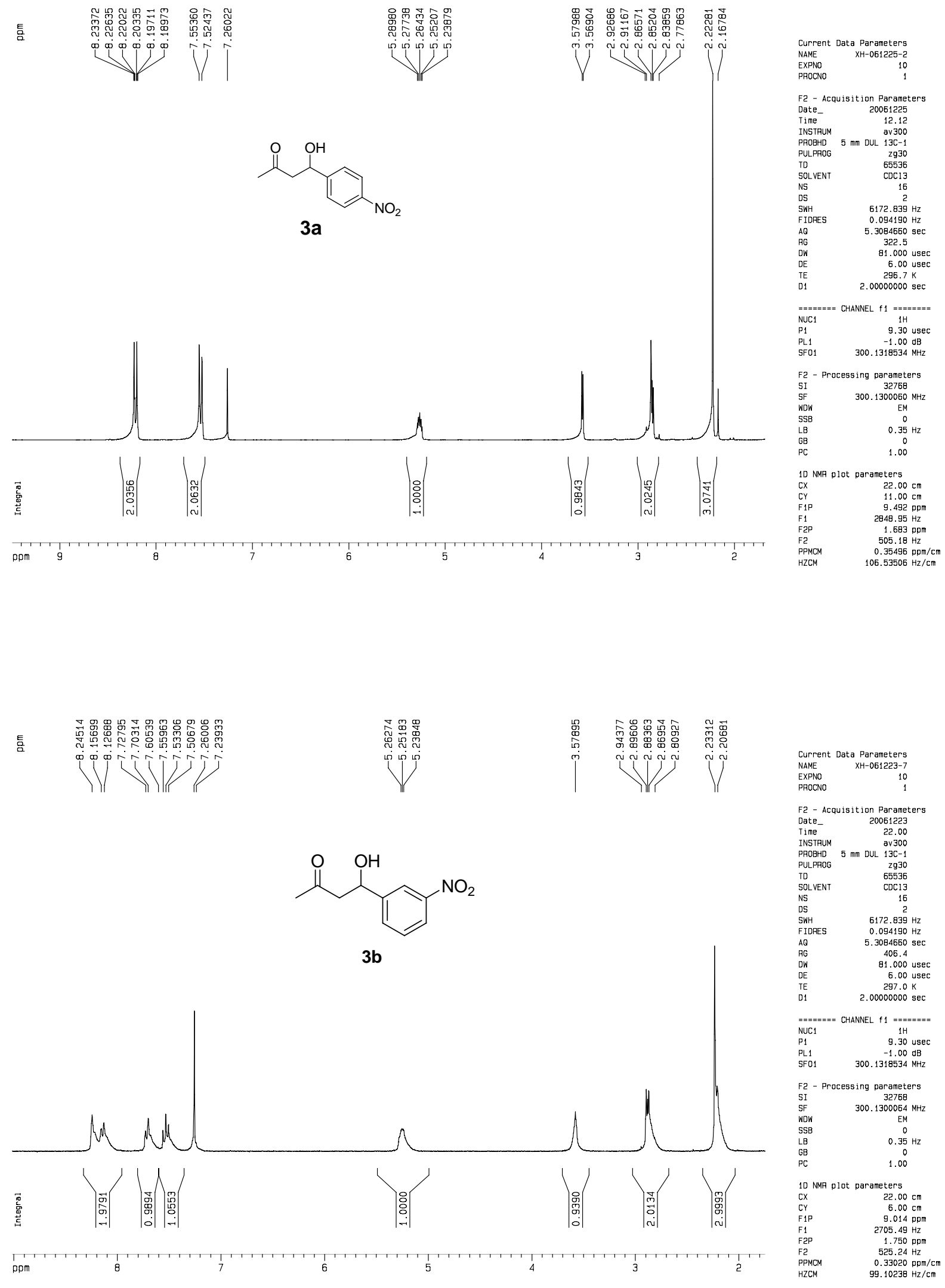

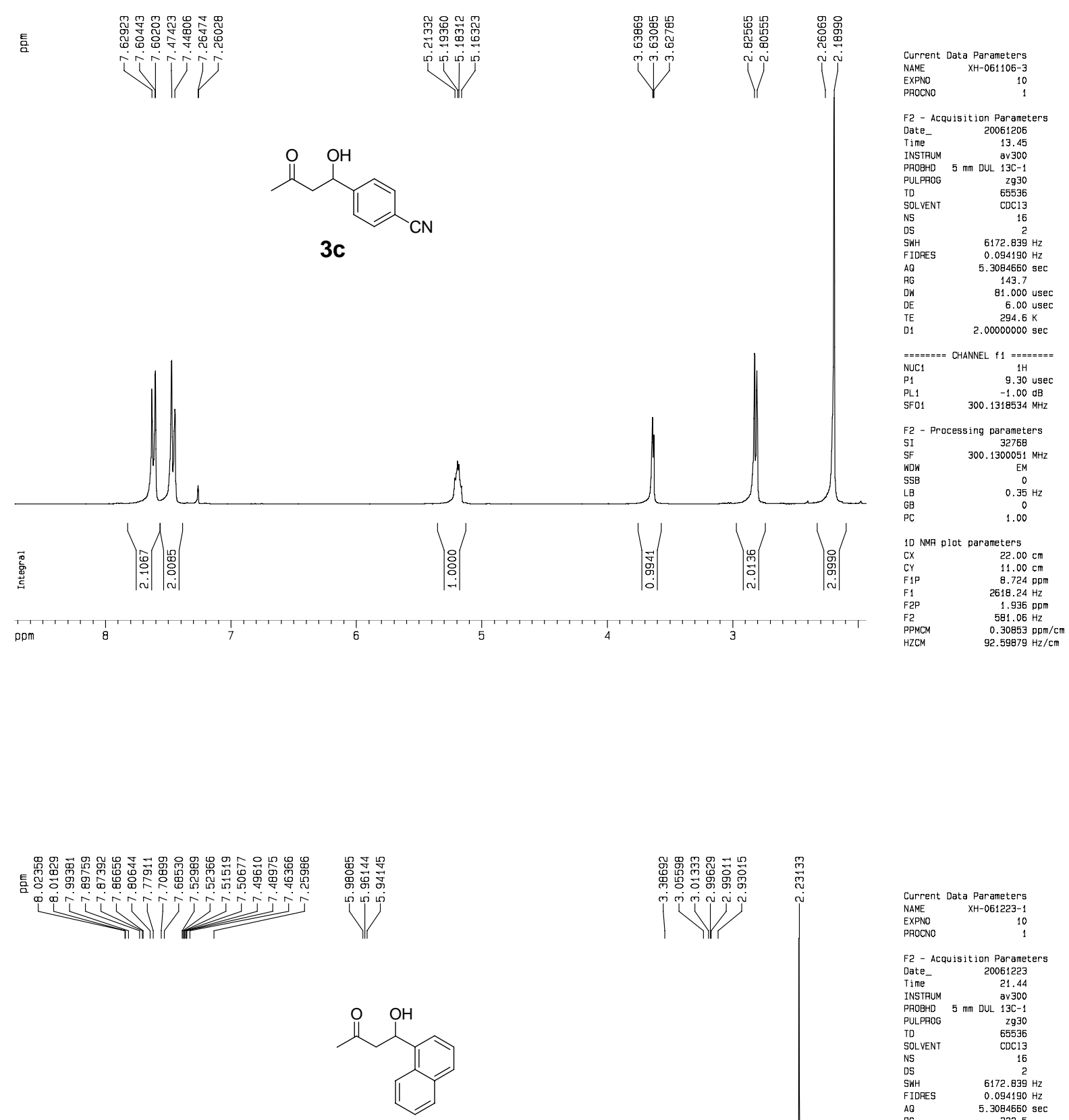

F2 - Acquisition Parameter

Date 20061223

$\begin{array}{ll}\text { Time } & 21.44 \\ \text { INSTRUM } & \text { av300 }\end{array}$

PROBHD $5 \mathrm{~mm}$ DUL $13 \mathrm{CC}-1$
PULPROG
2930
65536

$\begin{array}{lr}\text { TD } & 65536 \\ \text { SOLVENT } & \text { CDC13 } \\ \text { NS } & 16\end{array}$

$\begin{array}{lc}\text { DS } & 2 \\ \text { SWH } & 6172.839 \mathrm{~Hz} \\ \text { FTDRES } & 0.094190 \mathrm{~Hz}\end{array}$

$\begin{array}{ll}\text { FIDRES } & 0.094190 \mathrm{~Hz} \\ A Q & 5.3084660 \mathrm{sec}\end{array}$

3d

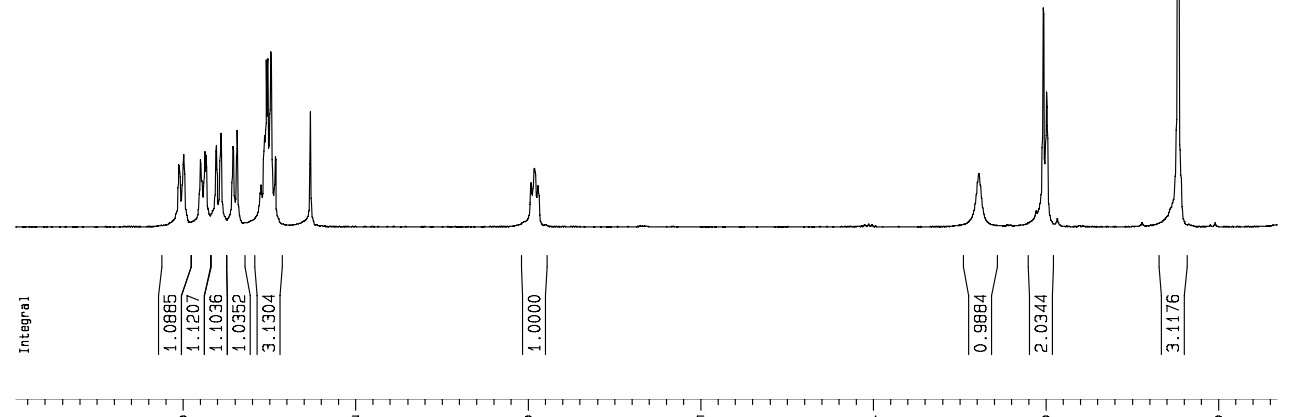

322.5
81.000 usec
6.00 usec

$297.1 \mathrm{~K}$

$=======$ CHANNEL $f 1=======$

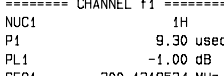

F2 - Processing parameters
SI

$\begin{array}{lc}\text { SI } & 32768 \\ \text { SF } & 300.1300060 \mathrm{MHZ} \\ \text { WOW } & \text { EM } \\ \text { SSB } & 0\end{array}$

$\begin{array}{lc}\text { SSB } & 0 \\ \mathrm{LB} & 0.35 \mathrm{~Hz} \\ \mathrm{~GB} & 0 \\ \mathrm{PC} & 1.00\end{array}$

10 NMA plot parameters

$\begin{array}{lc}\text { CX } & 22.00 \mathrm{~cm} \\ \text { CY } & 12.00 \mathrm{~cm} \\ \text { F1P } & 8.970 \mathrm{ppm} \\ \text { F1 } & 2692.24 \mathrm{~Hz} \\ \text { F2P } & 1.660 \mathrm{ppm} \\ \text { F2 } & 498.36 \mathrm{~Hz} \\ \text { PPMCM } & 0.33226 \mathrm{ppm} / \mathrm{cm} \\ \text { HZCM } & 99.72177 \mathrm{~Hz} / \mathrm{cm}\end{array}$ 

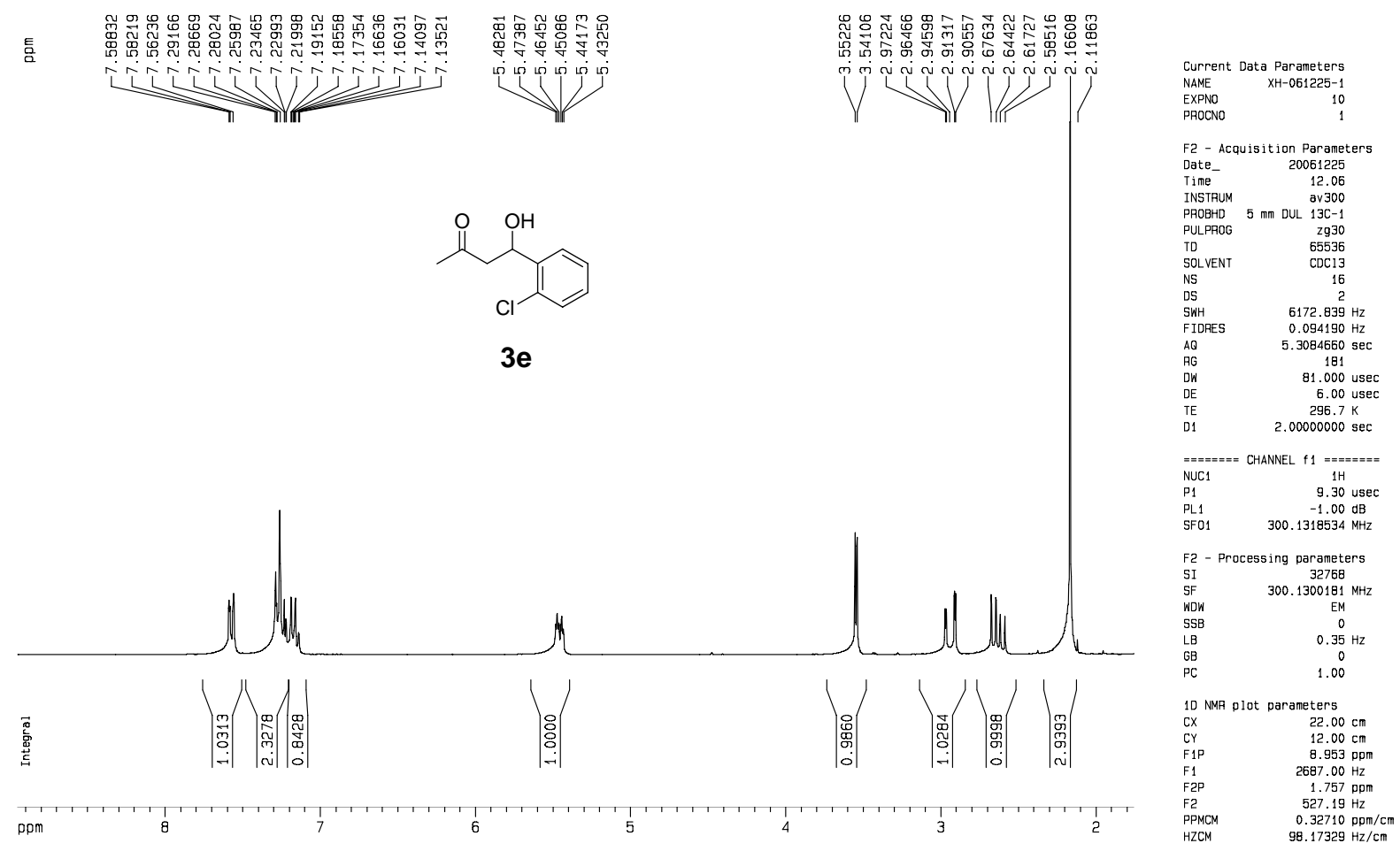

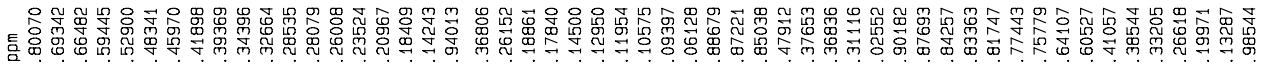

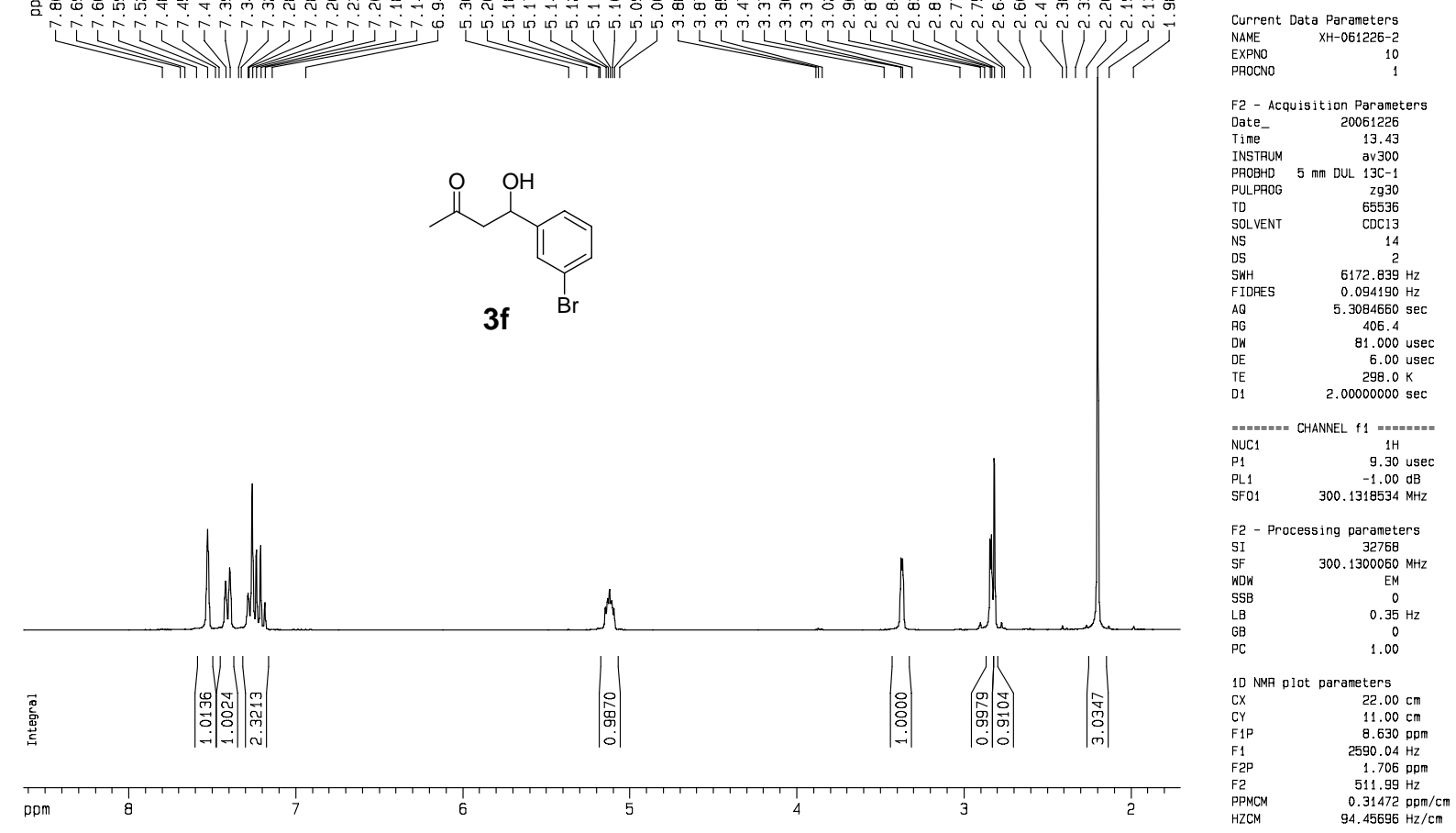

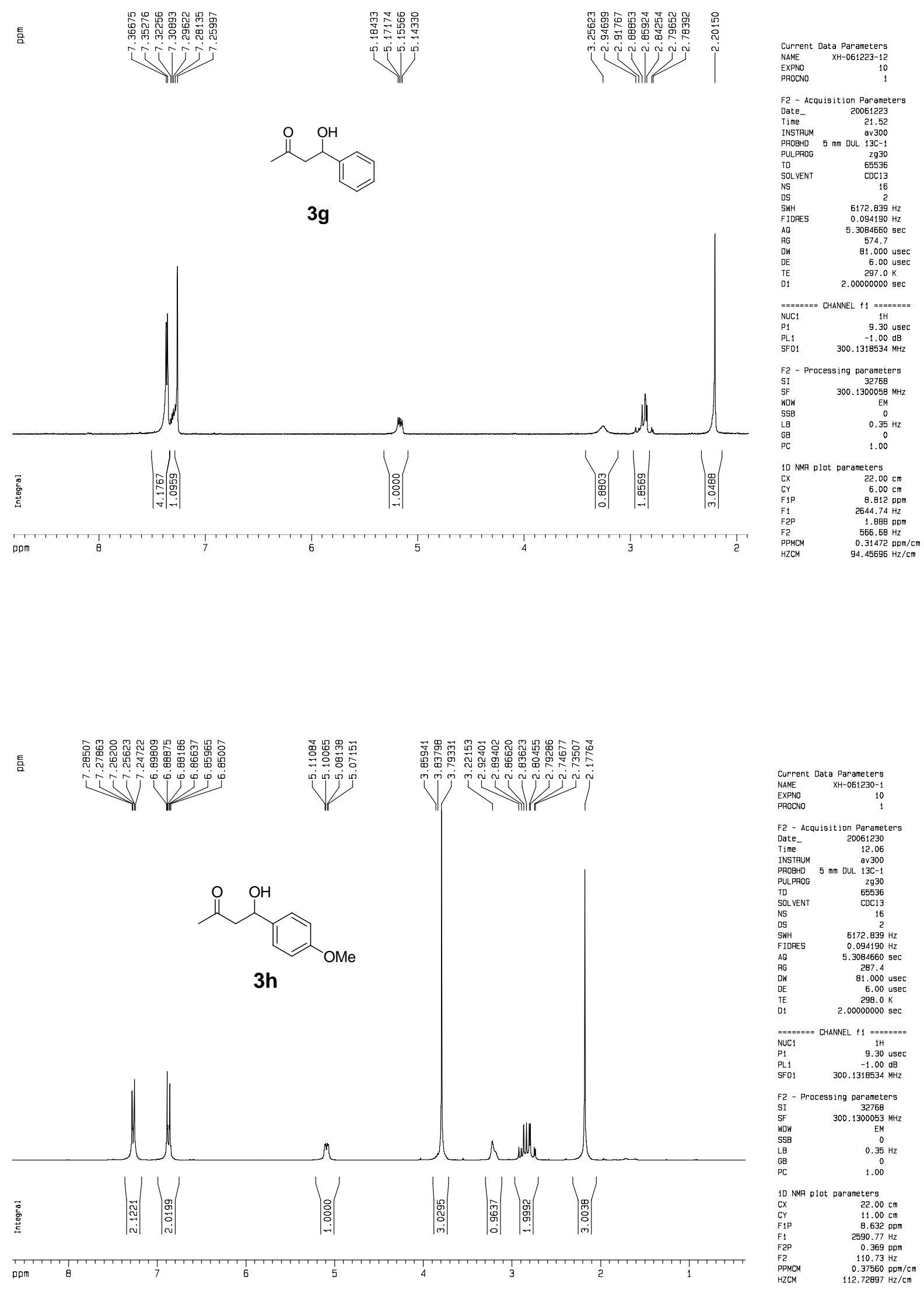

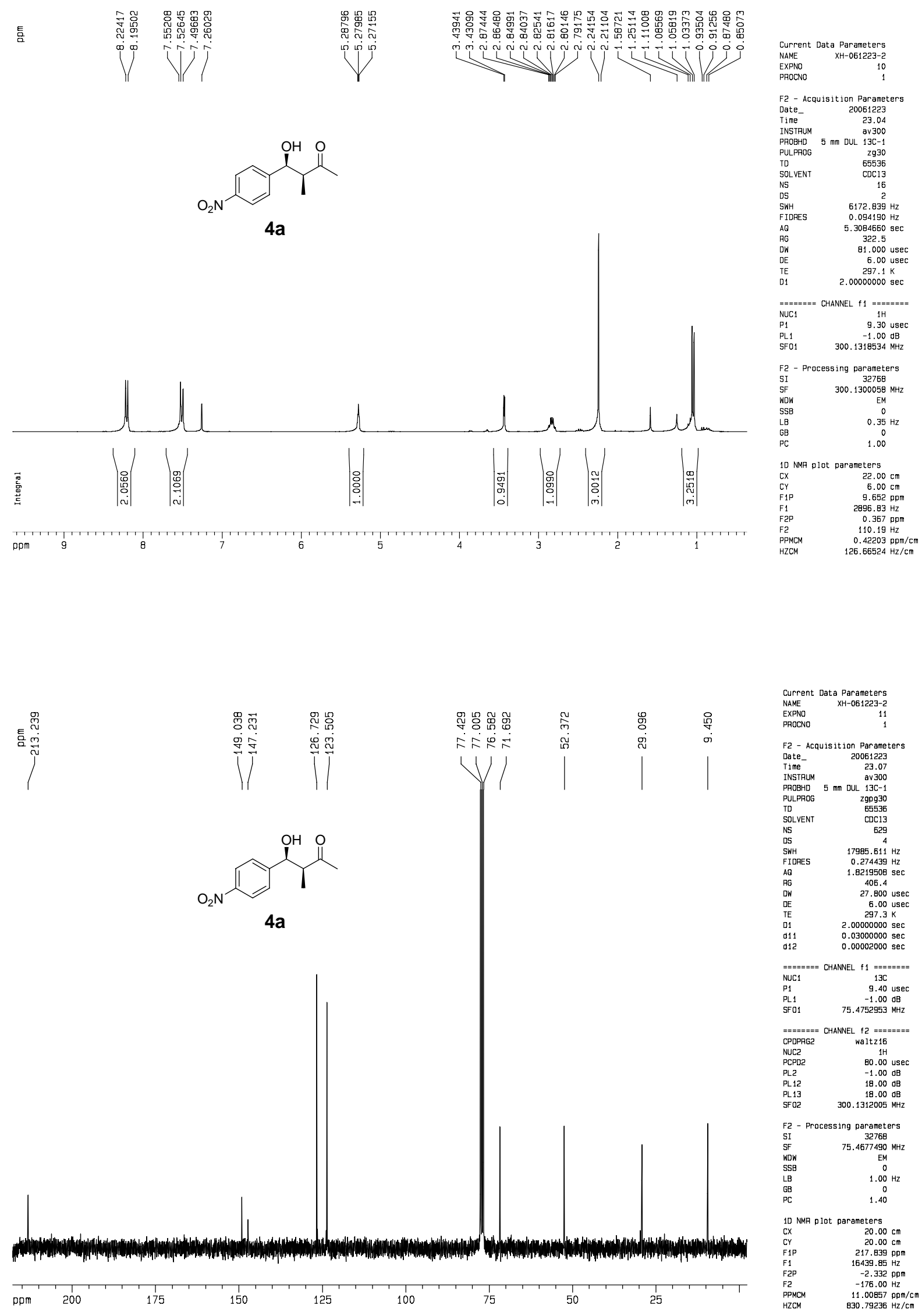

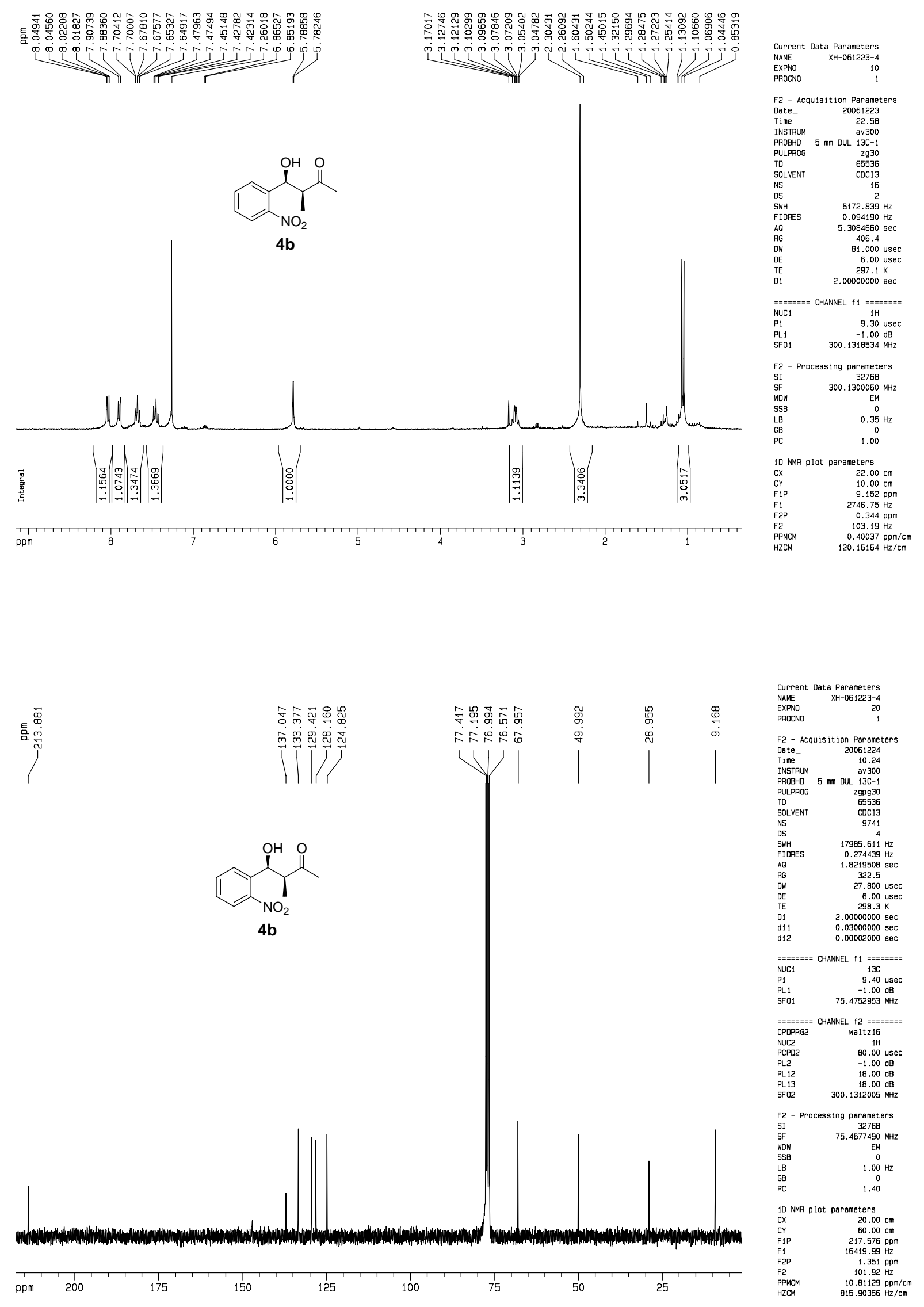

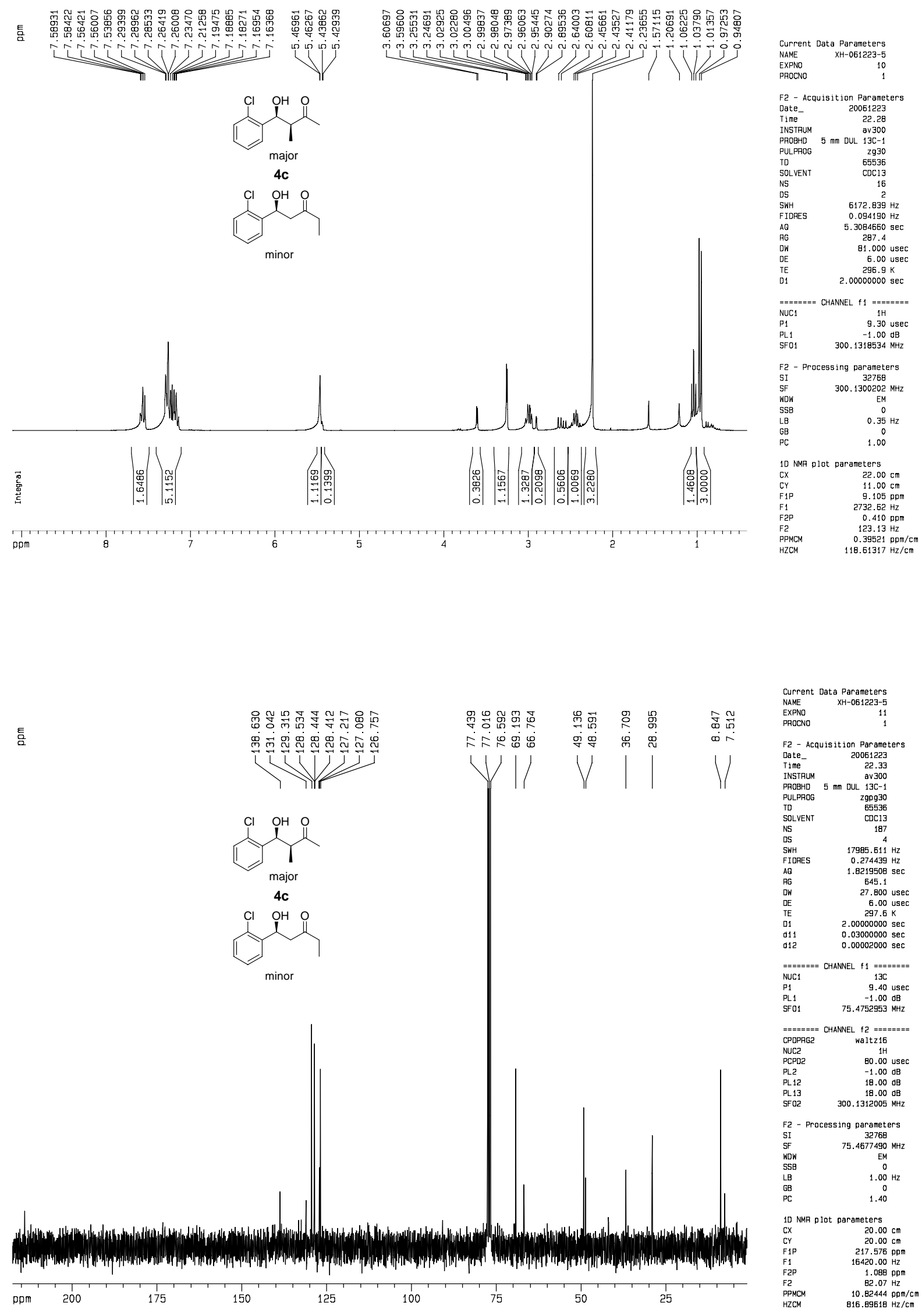

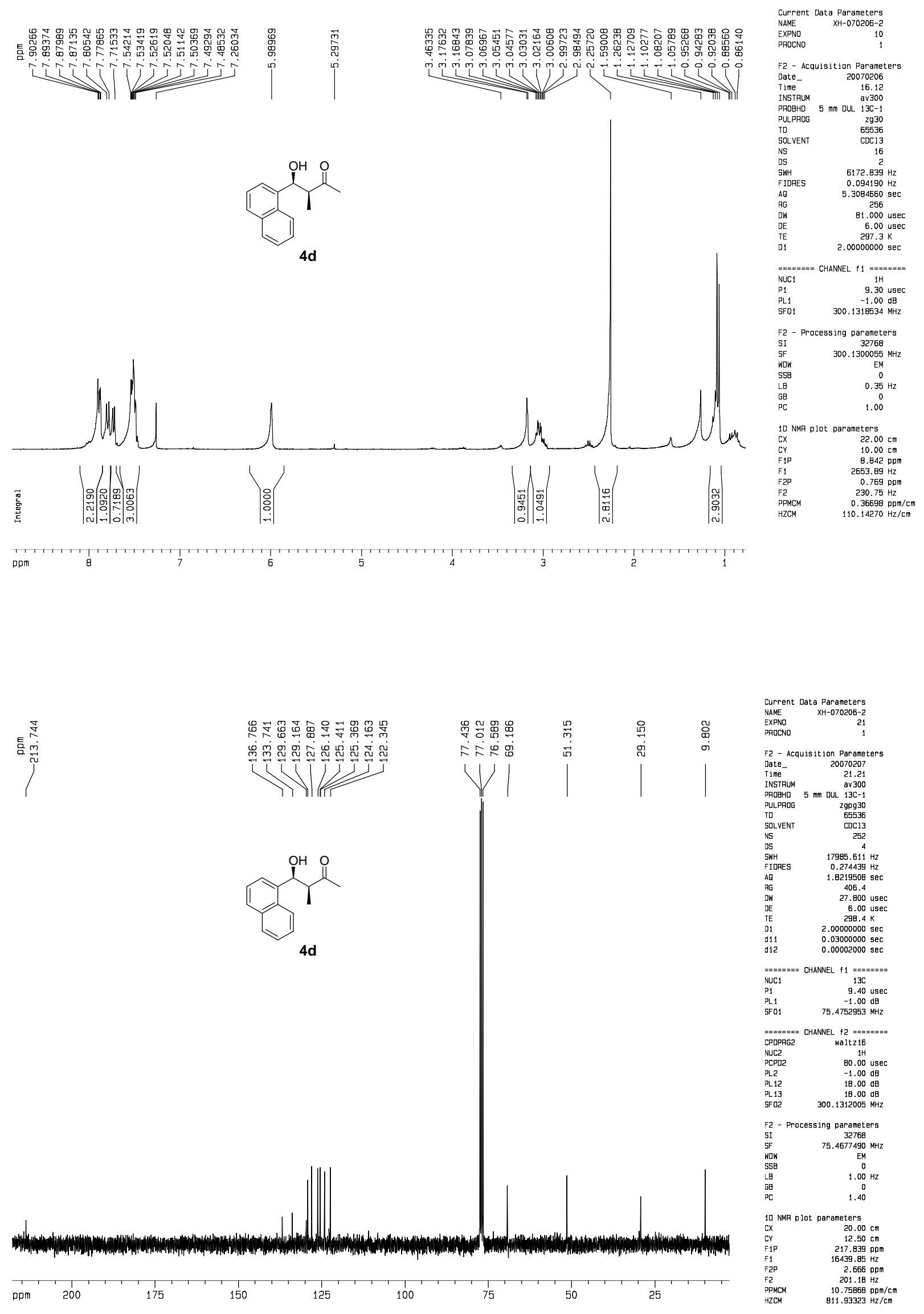

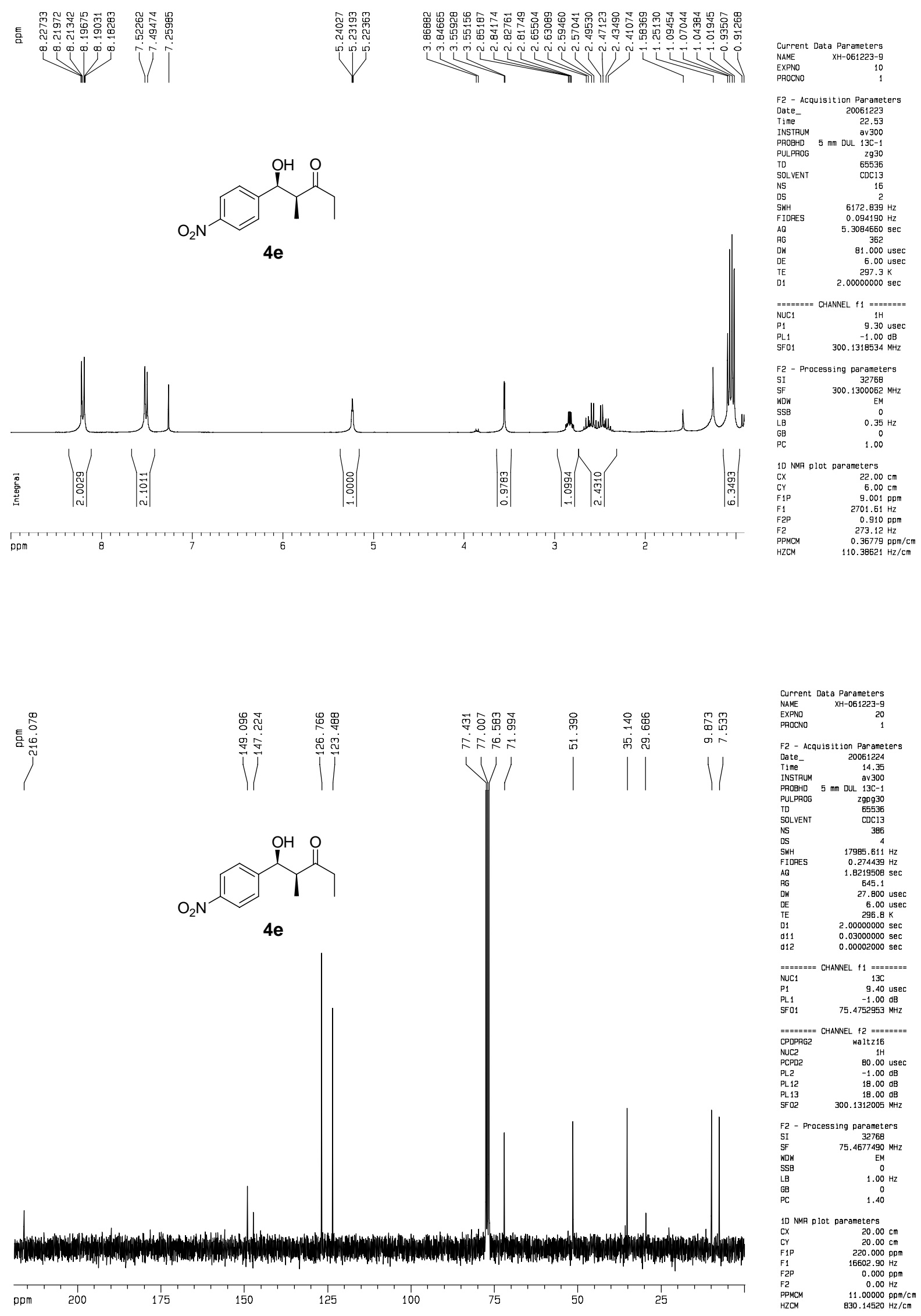

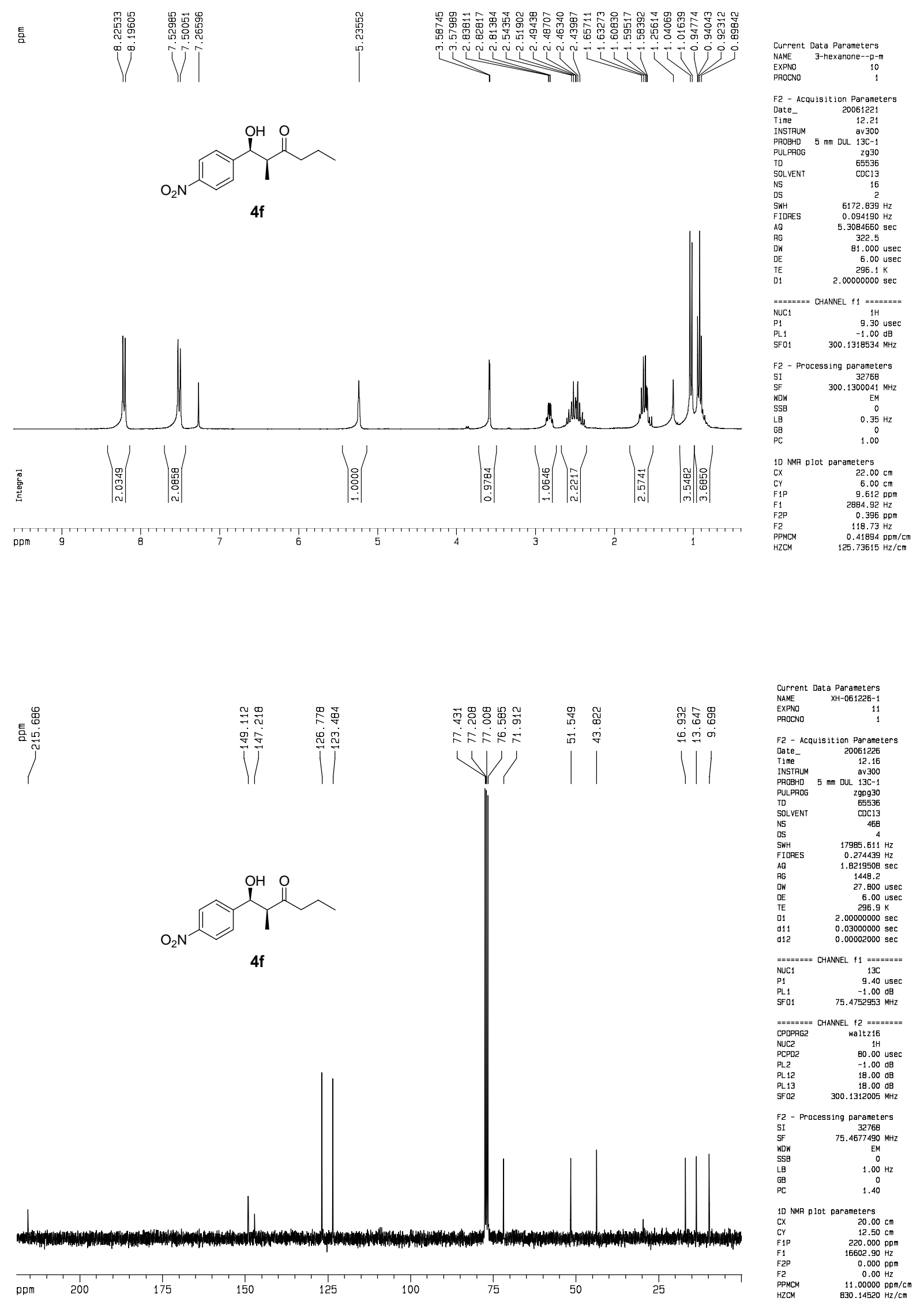


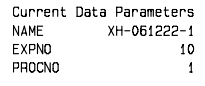

F2 - Acquisition Parameters
Date_ 20061222

av 300
PROBHD $5 \mathrm{~mm}$ DUL $13 \mathrm{C}-1$

$\begin{array}{lr}\text { PULPROG } & 2930 \\ \text { TD } & 69536\end{array}$

$\begin{array}{lr}\text { SOLVENT } & \mathrm{CDC} 13 \\ \text { NS } & 16 \\ \text { DS } & 2\end{array}$

$\begin{array}{ll}\text { SWH } & 6172.839 \mathrm{~Hz} \\ \text { IDDRES } & 0.094190 \mathrm{~Hz}\end{array}$

$\begin{array}{ll}A Q & 5.3084660 \mathrm{sec} \\ \mathrm{AQ} & 203.2\end{array}$

$\begin{array}{lr}\text { RG } & 203.2 \\ \text { OW } & 81.000 \text { use } \\ D E & 6.00 \text { use } \\ \text { DE } & 295.8 \mathrm{~K}\end{array}$

$=======$ CHANNEL $f 1======-$
NUC1

$\begin{array}{ll}\text { P1 } & 9.30 \text { usec } \\ \text { PL1 } & -1.00 \text { dB }\end{array}$

$\begin{array}{lr}\text { PL1 } & -1.00 \mathrm{~dB} \\ \text { SF01 } & 300.1318534 \mathrm{MHz}\end{array}$

F2 - Processing parameters
SI 32768
$300.1300047 \mathrm{MHZ}$

$\begin{array}{lc}\text { SF } & 300.1300047 \mathrm{MHz} \\ \text { WOW } & \text { EM } \\ \text { SSB } & 0 \\ \text { LB } & 0.35 \mathrm{~Hz} \\ \text { GB } & 0 \\ \text { PC } & 1.00\end{array}$

10 NMA plot parameters

$\begin{array}{lr}\text { 10 NMF plot parameters } \\ \text { CX } & 22.00 \mathrm{~cm} \\ \text { CY } & 5.00 \mathrm{~cm} \\ \text { FIP } & 9.202 \mathrm{ppm} \\ \text { F1 } & 2761.72 \mathrm{~Hz} \\ \text { F2P } & 1.915 \mathrm{ppm} \\ \text { F2 } & 574.66 \mathrm{~Hz} \\ \text { PPMCM } & 0.33123 \mathrm{ppm} / \mathrm{C} \\ \text { HZCM } & 99.41208 \mathrm{~Hz} / \mathrm{C}\end{array}$

pp

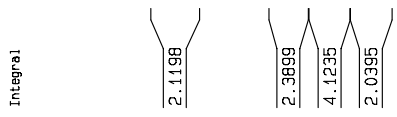

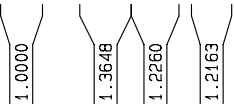
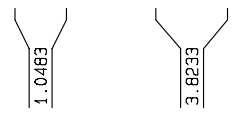

ppm

|cे

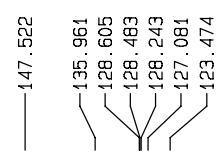

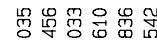

करำ兵星

111

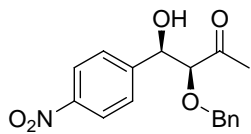

$4 g$

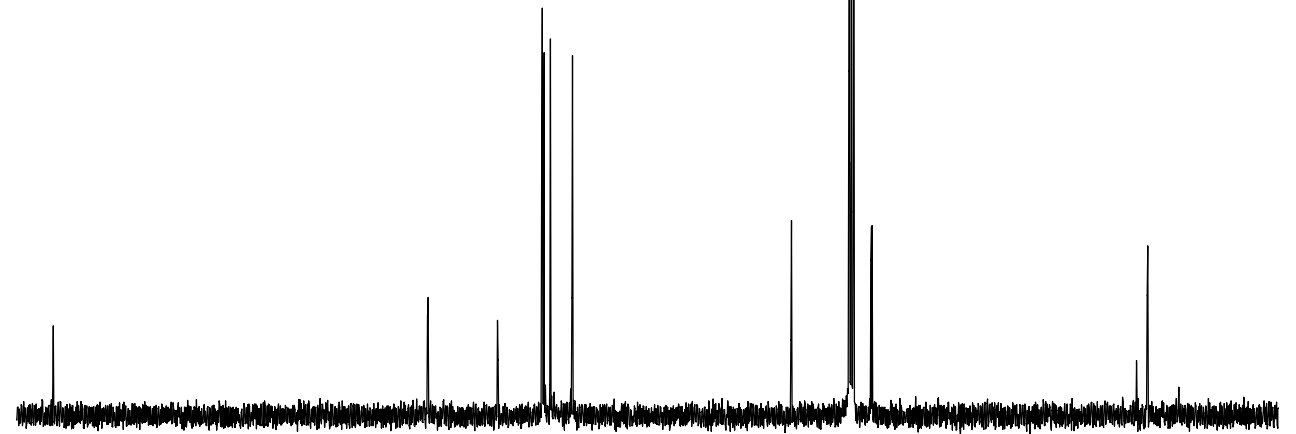

ppm

200

150

125

100

50

25

总

Current Data Parameters
NAME XH-061222-1

EXPNO
PROCNO

F2 - Acquisition Parameters
Date_ 20061222

$\begin{array}{lr}\text { Date } & 20061222 \\ \text { Time } & 12.51\end{array}$

INSTRUM $\quad$ av 300

29p930
PULPROG

\begin{tabular}{lr} 
ID & 65536 \\
SOLVENT & COC13 \\
NS & 860 \\
\hline
\end{tabular}

$\begin{array}{lr}\text { DS } & 860 \\ \text { SWH } & 47985.611 \mathrm{~Hz} \\ \text { I IDPES } & 0.274439 \mathrm{~Hz}\end{array}$

$\begin{array}{ll}0.274439 \mathrm{~Hz} \\ \text { IA } & 1.8219508 \mathrm{sec}\end{array}$

$\begin{array}{ll}A \mathrm{AB} & 1.8249508 \mathrm{sec} \\ \mathrm{FG} & 406.4 \\ \mathrm{WH} & 27.800 \mathrm{sec}\end{array}$

27.800 usec
6.00 usec

$\begin{array}{ll}01 & 2.00000000 \mathrm{sec} \\ \mathrm{d} & 0.03000000 \mathrm{sec}\end{array}$

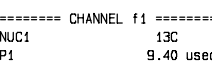

$\begin{array}{lr}\text { PL1 } & -1.00 \mathrm{~dB} \\ \text { SF01 } & 75.4752953 \mathrm{MHz}\end{array}$

$======$ CHANNEL
CPDPRG2 $2======$
waltz16

$\begin{array}{ll}\text { NUC2 } & 1 \mathrm{H} \\ \mathrm{PCPO2} & 80.00 \text { use }\end{array}$

$\begin{array}{ll}\text { PL2 } & -1.00 \mathrm{~dB} \\ \text { PL12 } & 18.00 \mathrm{~dB}\end{array}$

$\begin{array}{lr}18.00 \mathrm{~dB} \\ \mathrm{PL} 13 & 300.1312005 \mathrm{MHZ}\end{array}$

F2 - Processing parameters

$\begin{array}{ll}\mathrm{SI} & 32768 \\ \mathrm{SF} & 75.4677490 \mathrm{MHz}\end{array}$

$\begin{array}{cc}E M \\ \text { SSB } & 0 \\ B & 1.00 \mathrm{~Hz}\end{array}$

10 NMP plot parameters
CX $20.00 \mathrm{~cm}$

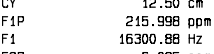

$\begin{array}{ll}F 2 \mathrm{P} & 6.085 \mathrm{ppm} \\ =2 & 459.25 \mathrm{~Hz}\end{array}$

$\begin{array}{lr}\text { PPMCM } & 10.49563 \mathrm{ppm} / \mathrm{c} \\ \text { HZCM } & 792.08160 \mathrm{~Hz} / \mathrm{cm}\end{array}$ 


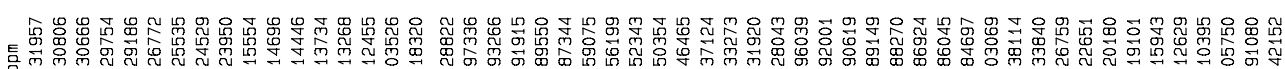

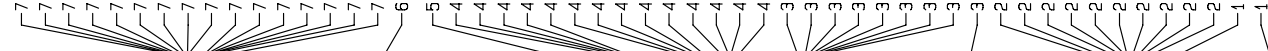<smiles>CC(=O)C(OCc1ccccc1)C(O)c1ccc(Cl)cc1</smiles>

4h
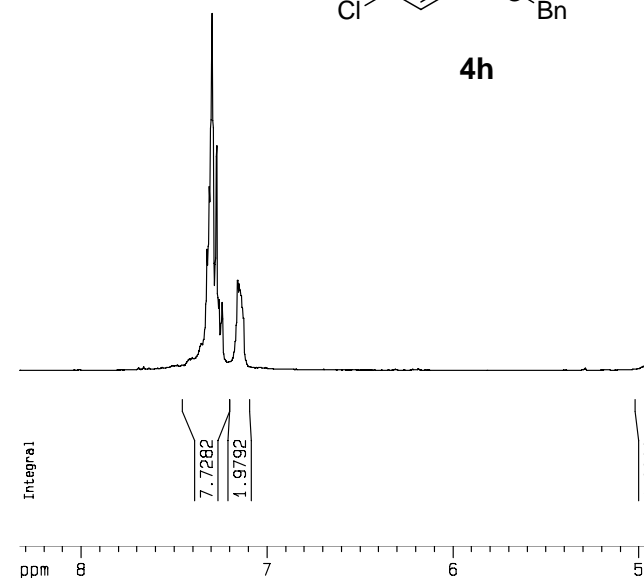

pom

|

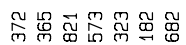

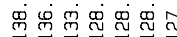

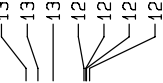

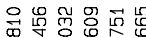

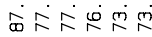

Wh

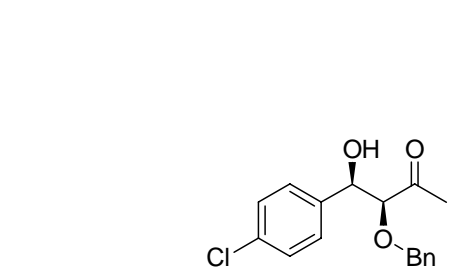

4h

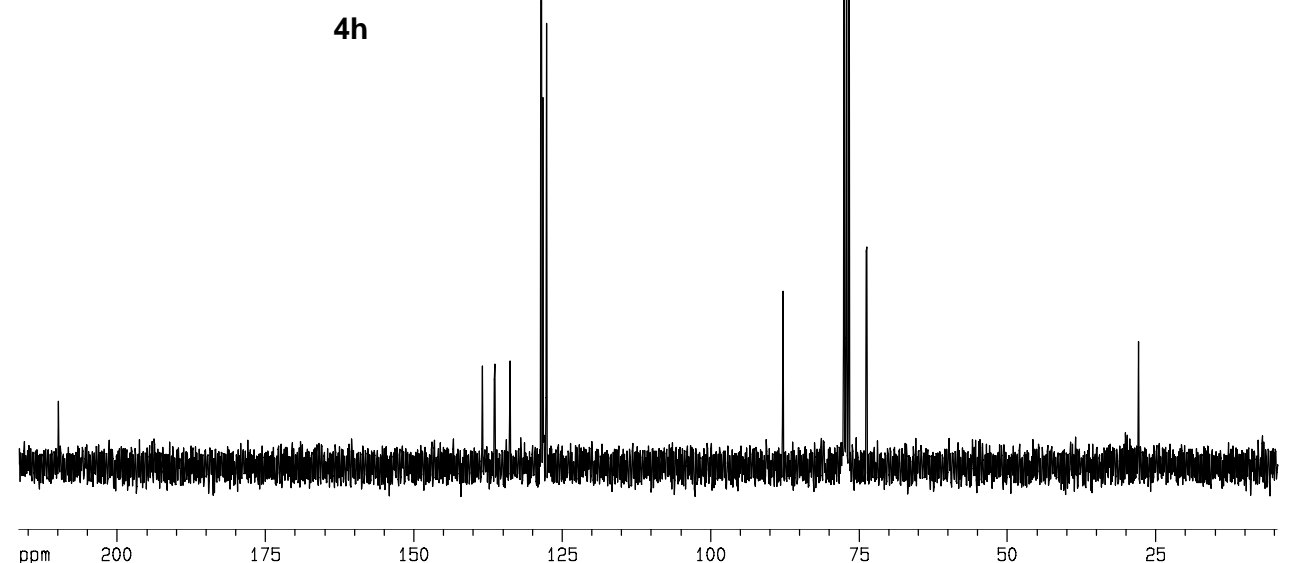

ppm 200
150

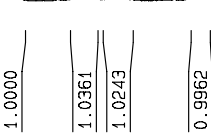

$\mid$

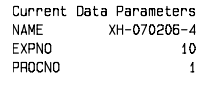

F2 - Acquisition Parameters

$\begin{array}{lr}\text { Date }_{-} & 20070206 \\ \text { Time } & 16.27\end{array}$

INSTRUM
PAOBHD $5 \mathrm{~mm}$ DUL $13 \mathrm{C} 300$
PUC-1

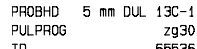

$\begin{array}{lr}\text { PULPPOG } & 2930 \\ \text { TD } & 65536 \\ \text { 50LVENT } & \text { CDC13 } \\ \text { NS } & 16\end{array}$

$\begin{array}{lr}\text { DS } & 2 \\ \text { 5WH } & 6172.839 \mathrm{~Hz}\end{array}$

FIDRES $\quad 0.094190 \mathrm{~Hz}$

$\begin{array}{ll}A B & 5.3084660 \mathrm{sec}\end{array}$

$\begin{array}{lr}\text { RG } & 203.2 \\ \text { OW } & 81.000 \text { usec } \\ \text { DE } & 6.00 \text { usec } \\ \text { TE } & 297.3 \mathrm{~K}\end{array}$

6.00 usec
$297.3 \mathrm{~K}$

$=======$ CHANNEL $f 1=======$
NUC1

$\begin{array}{lr}P_{1} & 9.30 \text { use } \\ P L 1 & -1.00 \mathrm{~dB} \\ \mathrm{~S} F 01 & 300.1318534 \mathrm{MHz}\end{array}$

F2 - Processing parameters

$\begin{array}{cc}\text { SI } & 32768 \\ \text { SF } & 300.1300076 \mathrm{MHZ}\end{array}$

$\begin{array}{lc}\text { WOW } & E M \\ 5 S B & 0 \\ L B & 0.35 \\ \text { Hz }\end{array}$

$\begin{array}{lr}\mathrm{GB} & 0 \\ \mathrm{PC} & 1.00\end{array}$

10 NMF plot parameters
CX $22.00 \mathrm{~cm}$

CY $\quad 10.00 \mathrm{~cm}$

$\begin{array}{lr}\text { F1P } & 8.329 \mathrm{ppm} \\ \text { F1 } & 2499.92 \mathrm{~Hz} \\ \text { F2P } & 1.406 \mathrm{pH}\end{array}$

F2 $421.87 \mathrm{~Hz}$

$\begin{array}{lr}\text { PPMCM } & 0.31472 \mathrm{ppm} / \mathrm{cm} \\ \text { HZCM } & 94.45696 \mathrm{~Hz} / \mathrm{cm}\end{array}$

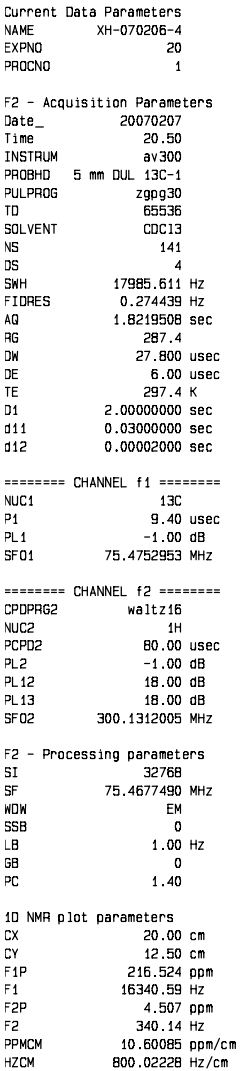




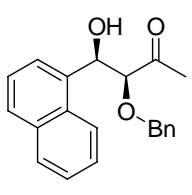

$4 i$
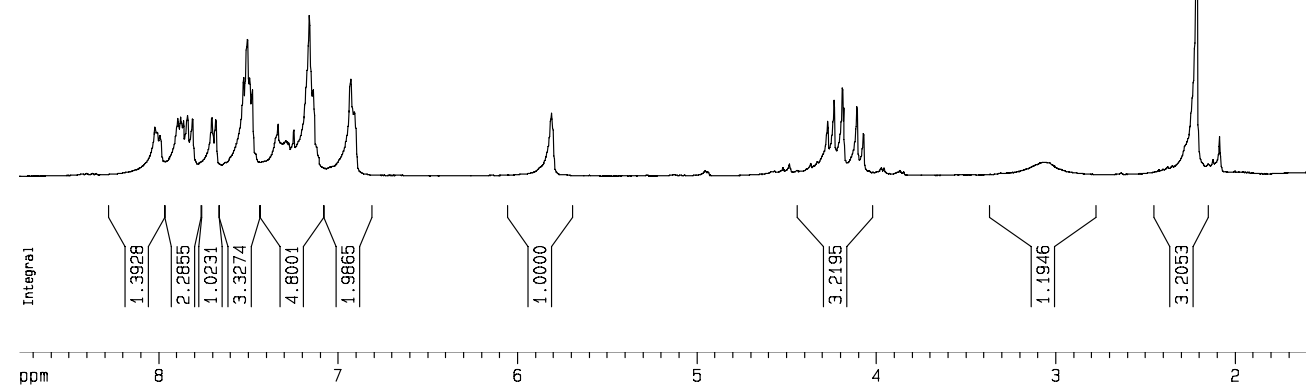

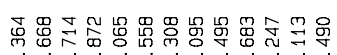

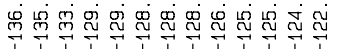
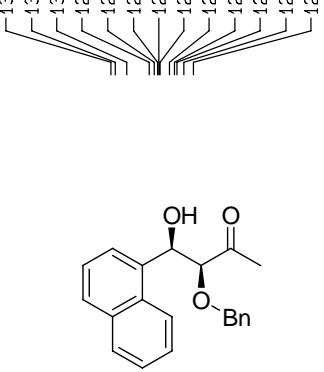

$4 i$

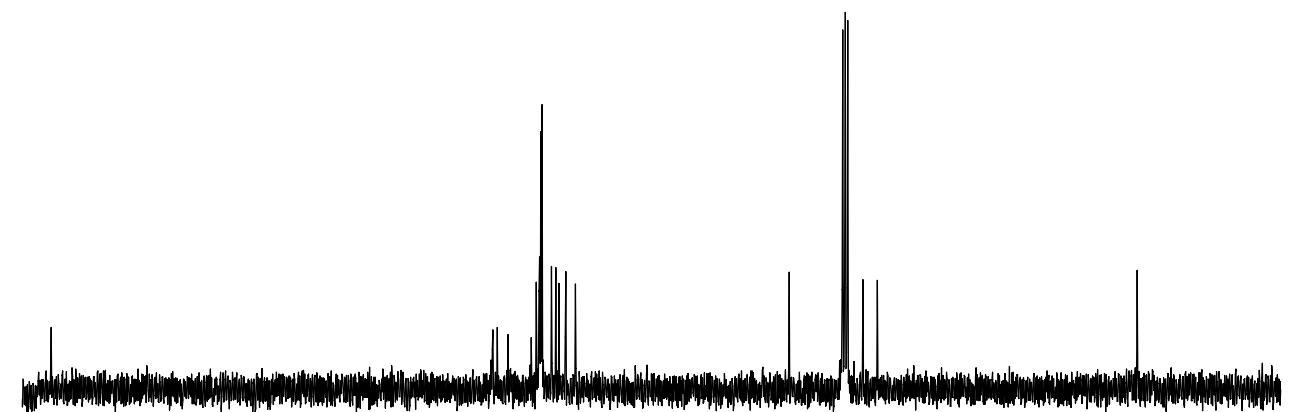

pp

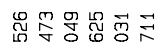

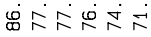

$11 / 1$

兽

กิ

25
- Acquisition Parame

INSTRUM av300

PAOBHD $5 \mathrm{~mm}$ DUL $13 \mathrm{C}-1$
PULPROG
2930

$\begin{array}{lr}2930 \\ \text { TD } & 65536 \\ \text { 5OLVENT } & \text { CDC13 }\end{array}$

16
DS
2

$\begin{array}{ll}\text { SWH } & 6172.839 \mathrm{~Hz} \\ \text { FIDRES } & 0.094190 \mathrm{~Hz}\end{array}$

AQ $\quad 5.3084650 \mathrm{sec}$

$\begin{array}{lr}\text { RG } & 181 \\ \text { DW } & 81.000 \text { usec } \\ \text { DE } & 6.00 \text { usec }\end{array}$

2. $00000000 \mathrm{sec}$

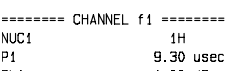

$\begin{array}{lr}\text { P1 } & 9.30 \text { use } \\ \text { PL1 } & -1.00 \mathrm{~dB} \\ \text { SF01 } & 300.1318534 \mathrm{MHz}\end{array}$

F2 - Processing parameters

$\begin{array}{ll}\mathrm{SI} & 32768 \\ \mathrm{SF} & 300.1300099 \mathrm{MHZ}\end{array}$

$\begin{array}{cc}\mathrm{NOW} & \mathrm{EM} \\ \mathrm{SSB} & 0 \\ \mathrm{~B} & 0.35 \mathrm{~Hz}\end{array}$

$\begin{array}{lr}\mathrm{GB} & 0 \\ \mathrm{PC} & 1.00\end{array}$

10 NMP plot parameters
$\mathrm{CX}$
$22.00 \mathrm{~cm}$

$\begin{array}{lr}\text { CY } & 62.00 \mathrm{~cm} \\ \mathrm{CY} & 6.776 \mathrm{pp} \\ \mathrm{F} 1 \mathrm{P} & 8.60 \mathrm{Fm}\end{array}$

$\quad 8.776 \mathrm{ppm}$
$=1$

$\begin{array}{lc}\text { F2 } & 1.602 \mathrm{ppm} \\ \text { FPMCM } & 480.83 \mathrm{~Hz} \\ \text { P } & 0.32607 \mathrm{ppm} / \mathrm{cm} \\ \text { HZCM } & 97.86360 \mathrm{~Hz} / \mathrm{cm}\end{array}$
Current Data Parameters
VAME XH-070206-

20

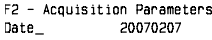

$\begin{array}{lr}\text { Time } & 16.14 \\ \text { INSTRUM } & \text { av300 } \\ \text { THOBHD } & 5 \mathrm{~mm} \text { DUL } 13 \mathrm{C}-1\end{array}$

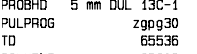

$\begin{array}{lr}\text { SOLVENT } & \text { COC13 } \\ \text { VS } & 81 \\ \text { JS } & 4\end{array}$

$\begin{array}{lr}\text { JWH } & 17985.611 \mathrm{~Hz} \\ =\text { IDRES } & 0.274439 \mathrm{~Hz}\end{array}$

$1.821450 \mathrm{~s}$

$\begin{array}{rr}\mathrm{JW} & 27.800 \mathrm{usec} \\ \mathrm{JE} & 6.00 \mathrm{usec} \\ \mathrm{JE} & 2.05 \mathrm{~K}\end{array}$

$\begin{array}{ll}11 & 0.03000000 \mathrm{sec} \\ 112 & 0.00002000 \mathrm{sec}\end{array}$

$=======$ CHANNEL $f 1=======$
vUC1 $13 \mathrm{C}$

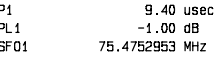

$=== \pm==$ CHANNEL $f 2=== \pm==3$

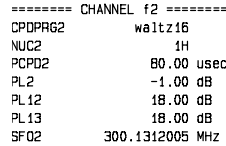

$\begin{array}{lr}=2 \text { - Processing parameters } \\ \text { SI } \\ \text { SF } & 75.46768 \\ \text { SF } & \end{array}$

$\begin{array}{lc}\text { HOW } & \text { EM } \\ \text { SSB } & 0 \\ -B & 1.00 \mathrm{~Hz} \\ \text { JB } & 0 \\ \text { JC } & 0\end{array}$

10 NMA plot parameters

$\begin{array}{lr}c X & 20.00 \mathrm{~cm} \\ c Y & 5.00 \mathrm{~cm}\end{array}$

$\begin{array}{ll}=1 \mathrm{P} & 215.472 \mathrm{ppm} \\ =1 & 16261.18 \mathrm{~Hz}\end{array}$

$\begin{array}{lr}=2 \mathrm{p} & 3.981 \mathrm{ppm} \\ =2 & 300.44 \mathrm{~Hz}\end{array}$

$\begin{array}{lr}\text { JPMCM } & 10.57454 \mathrm{ppm} / \mathrm{cm} \\ \text { HZCM } & 798.03711 \mathrm{~Hz} / \mathrm{cm}\end{array}$ 


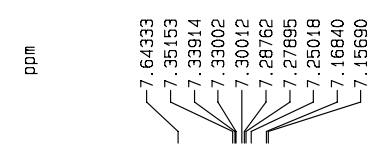

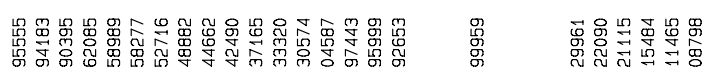
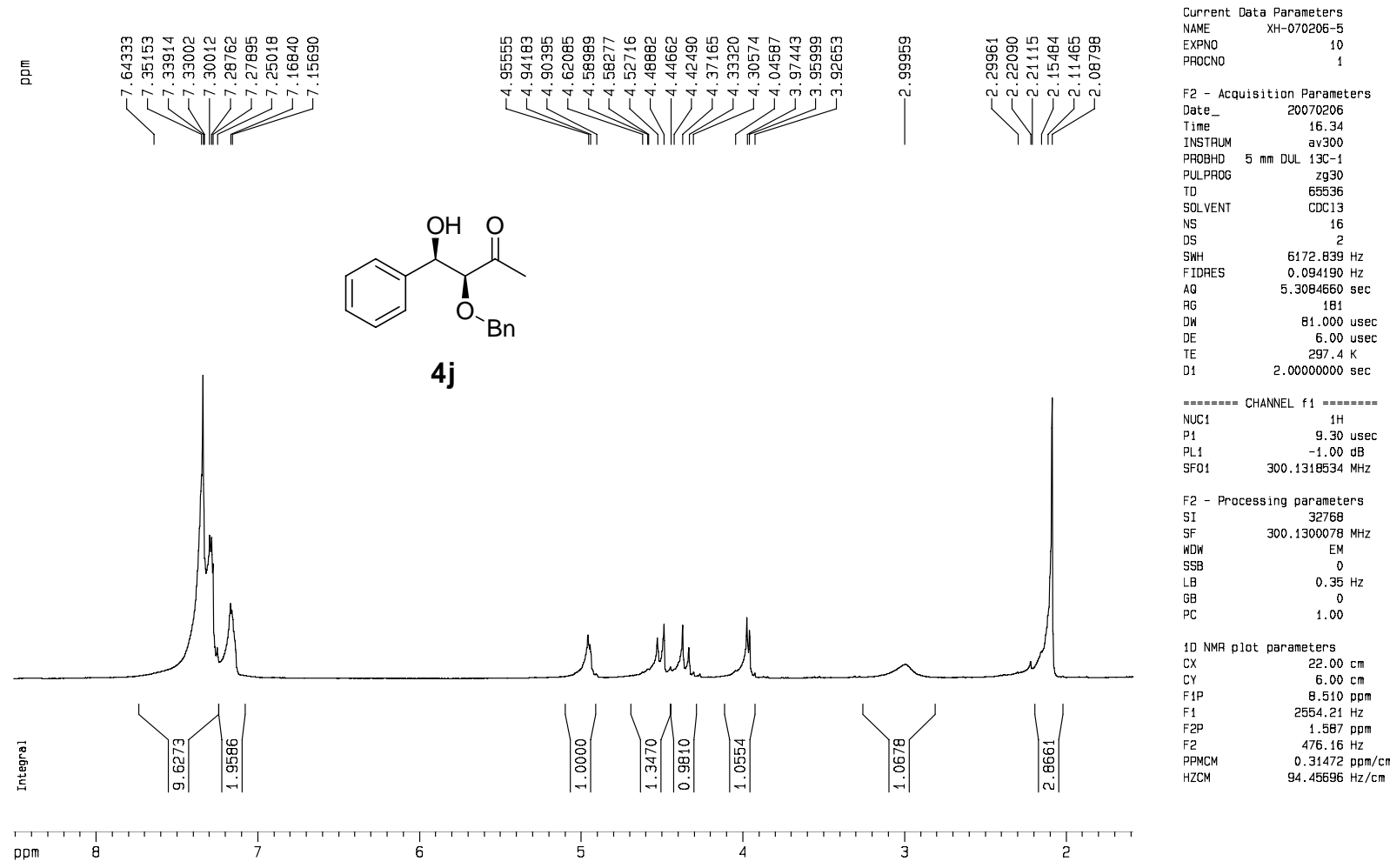

4j

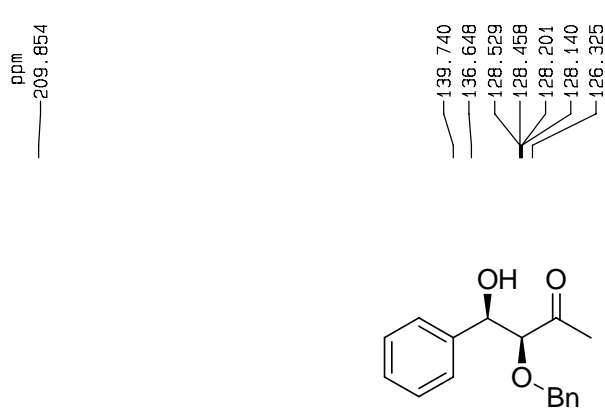

$4 \mathrm{j}$

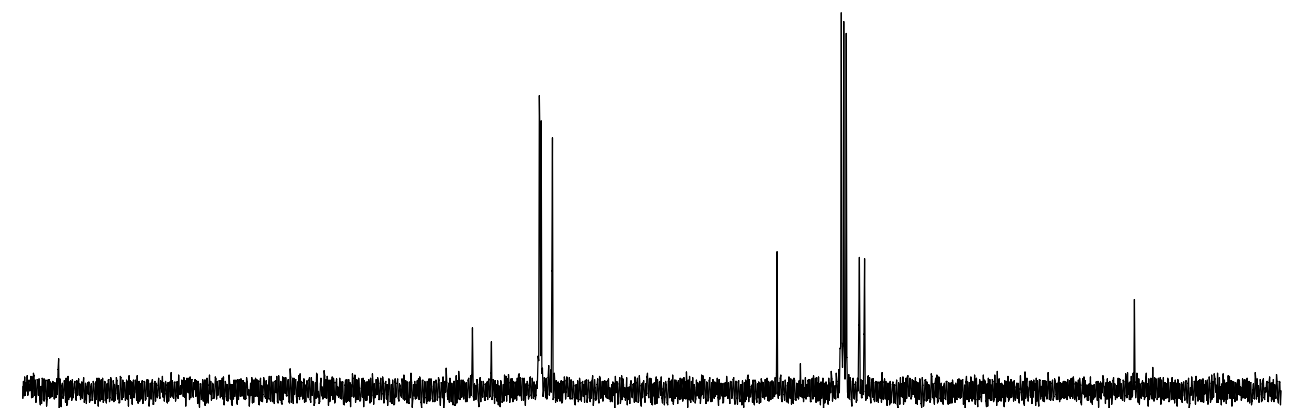

ppm

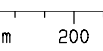

175

150

125

100

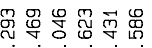

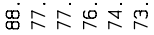

1W

ลิ 

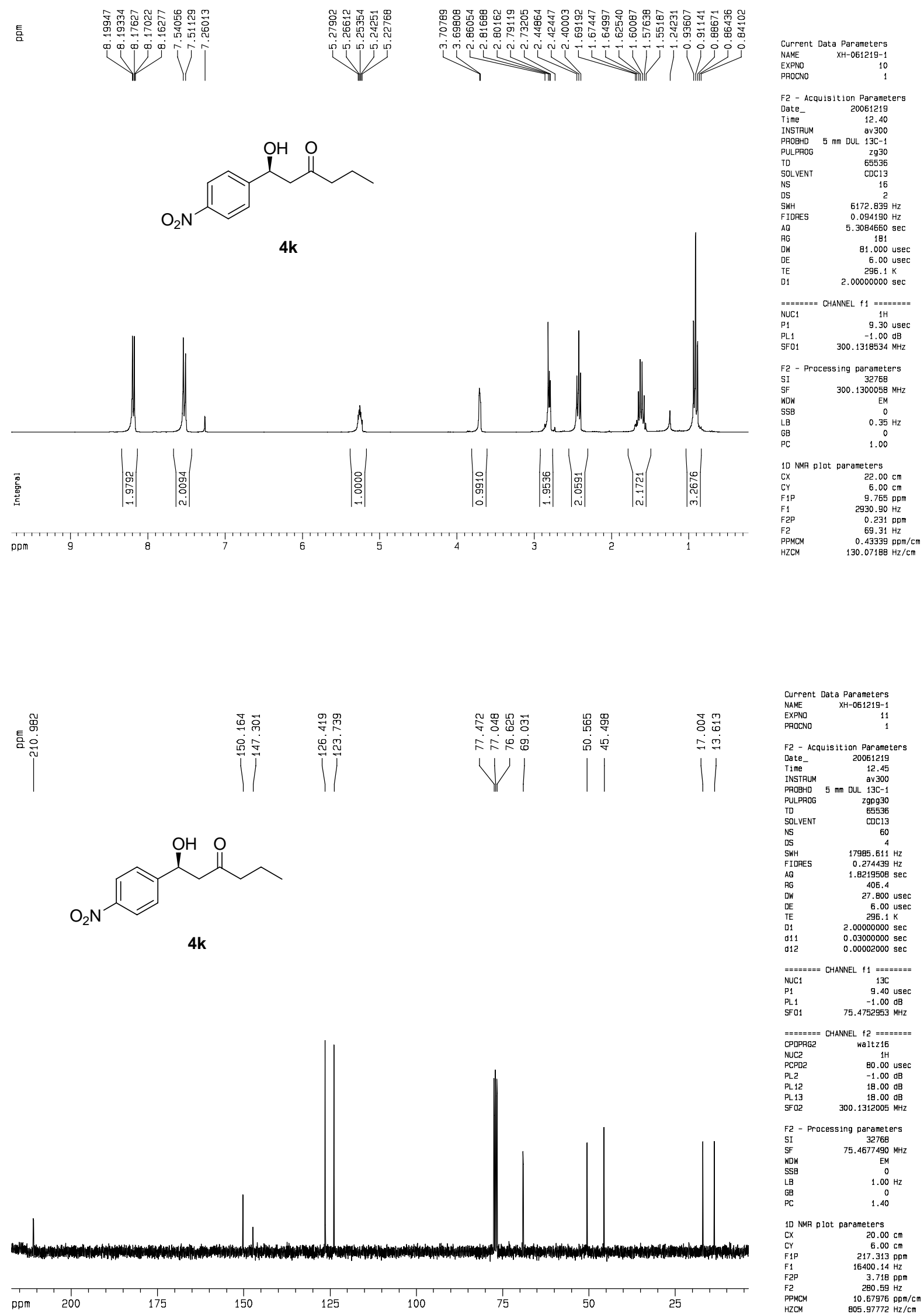

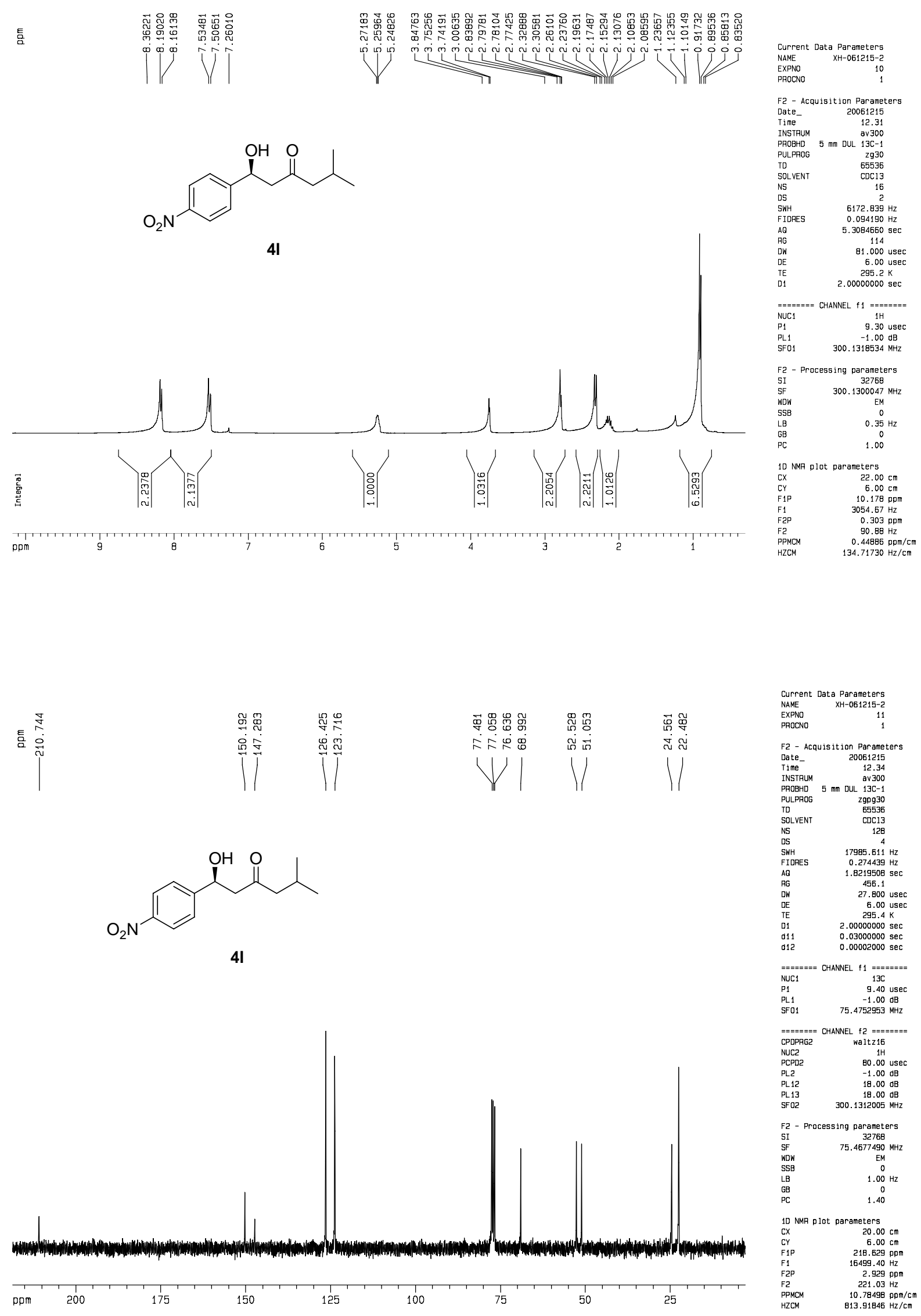


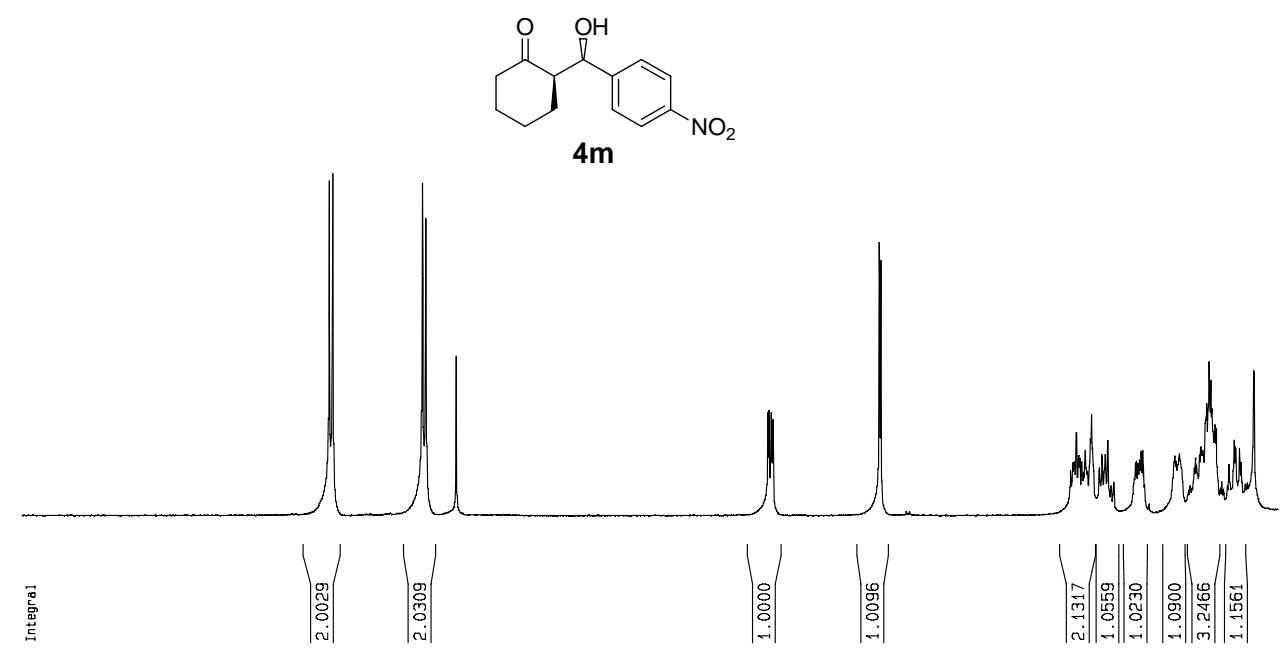

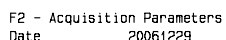

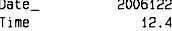

INSTHOM

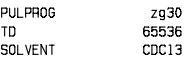

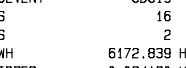

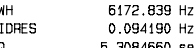

40
76

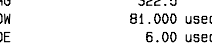

81.000 usec
6.00 usec
$297.4 \mathrm{~K}$

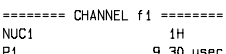

$\begin{array}{ll}-1.00 \mathrm{~dB} \\ \text { SF01 } & 300.1318534 \mathrm{MHz}\end{array}$

F2 - Processing parameters

$\begin{array}{lc}\text { SI } & 32768 \\ \text { SF } & 300.1300060 \mathrm{MHz} \\ \text { NOW } & \text { EM } \\ \text { SSB } & 0\end{array}$

SS
GB
$\triangle \mathrm{B}$

10 NMP plot parameters
CX $22.00 \mathrm{~cm}$
$\mathrm{CY}$
CY

$\begin{array}{lr}\mathrm{CY} & 6.00 \mathrm{~cm} \\ F 1 \mathrm{P} & 10.537 \mathrm{ppm} \\ =1 & 3162.36 \mathrm{~Hz} \\ =2 \mathrm{P} & 1.070 \mathrm{ppm}\end{array}$

$\begin{array}{ll}25 & 1.070 \mathrm{ppm} \\ \text { FPMCM } & 321.22 \mathrm{~Hz}\end{array}$

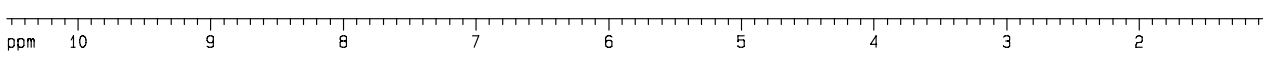

$\begin{array}{lr}0.43029 \mathrm{ppm} / \mathrm{cm} \\ \mathrm{TCMCM} & 129.14279 \mathrm{~Hz} / \mathrm{cm}\end{array}$
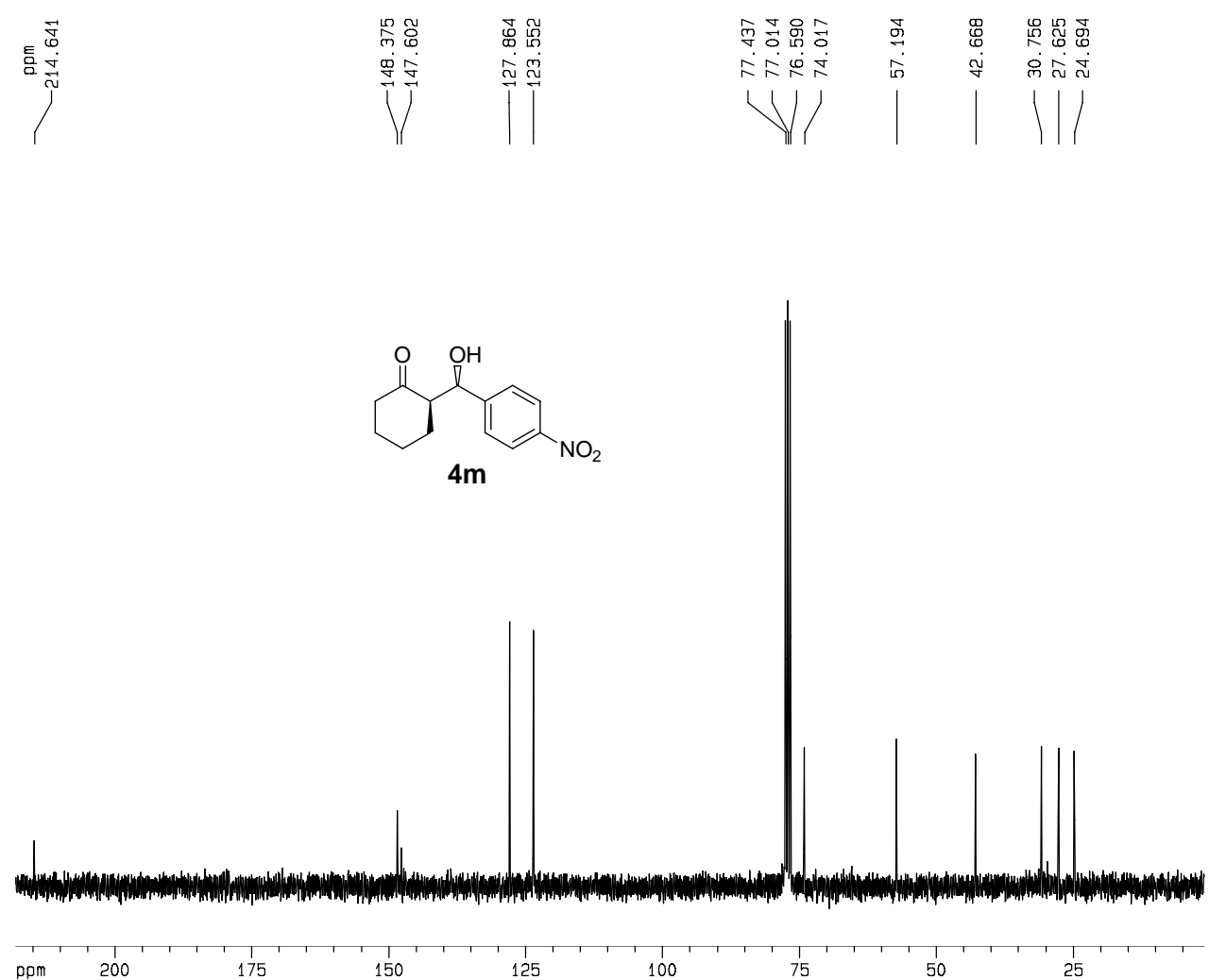

150

125

100

50

25

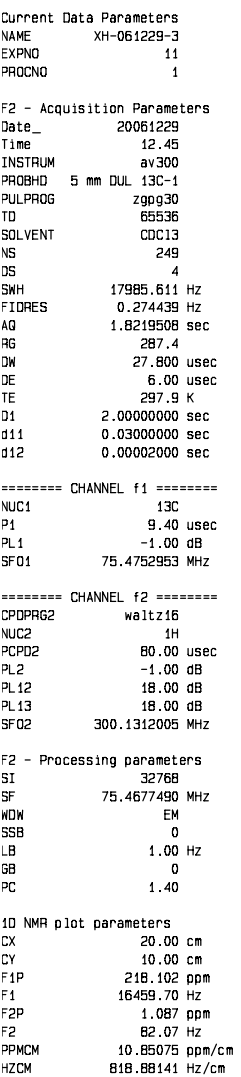




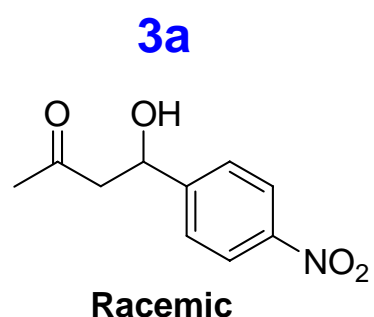

Sample ID : AS-H/3:7/0.5/25/254

User Name : System

Filename $\quad:$ E: $1429 \backslash x h \backslash 061227 \backslash$ racemic-acetone-pNO2

Method Name : E: $\mid g c h l \backslash m-4 . m e t$

Sequence Name: \{Sequence Name\}

Run Time $\quad$ :06-12-27 21:09:11

\section{Data Graph}

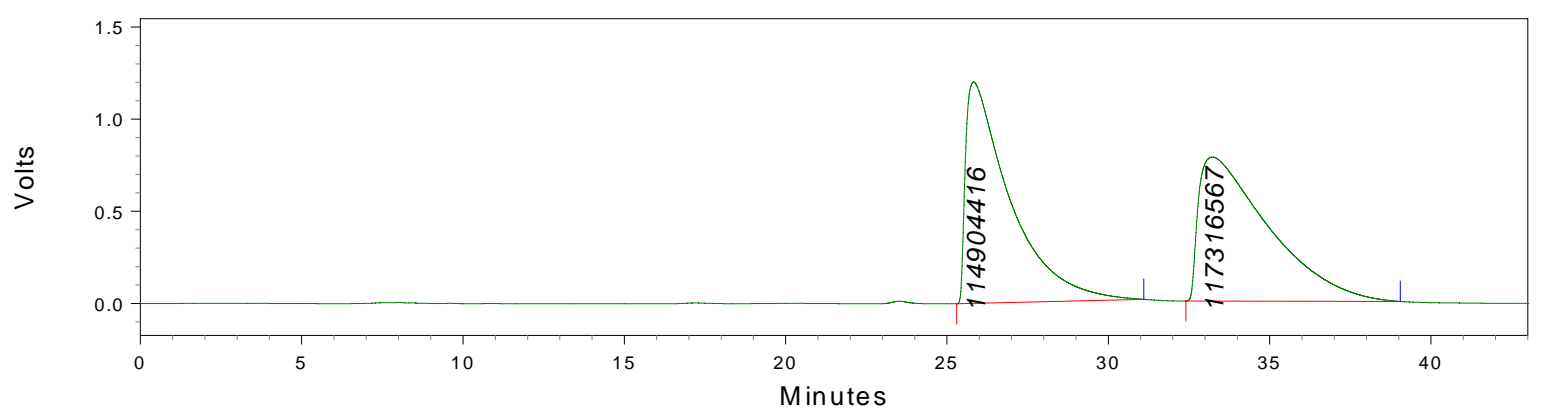

\section{Run Report}

Detector A (254nm)

Retention Time

Area

Area Percent

\begin{tabular}{|c|c|c|}
\hline \multicolumn{1}{|c|}{114904416} & 49.48 \\
33.219 & 117316567 & 50.52 \\
\hline Totals & & \\
& 232220983 & 100.00 \\
\hline
\end{tabular}




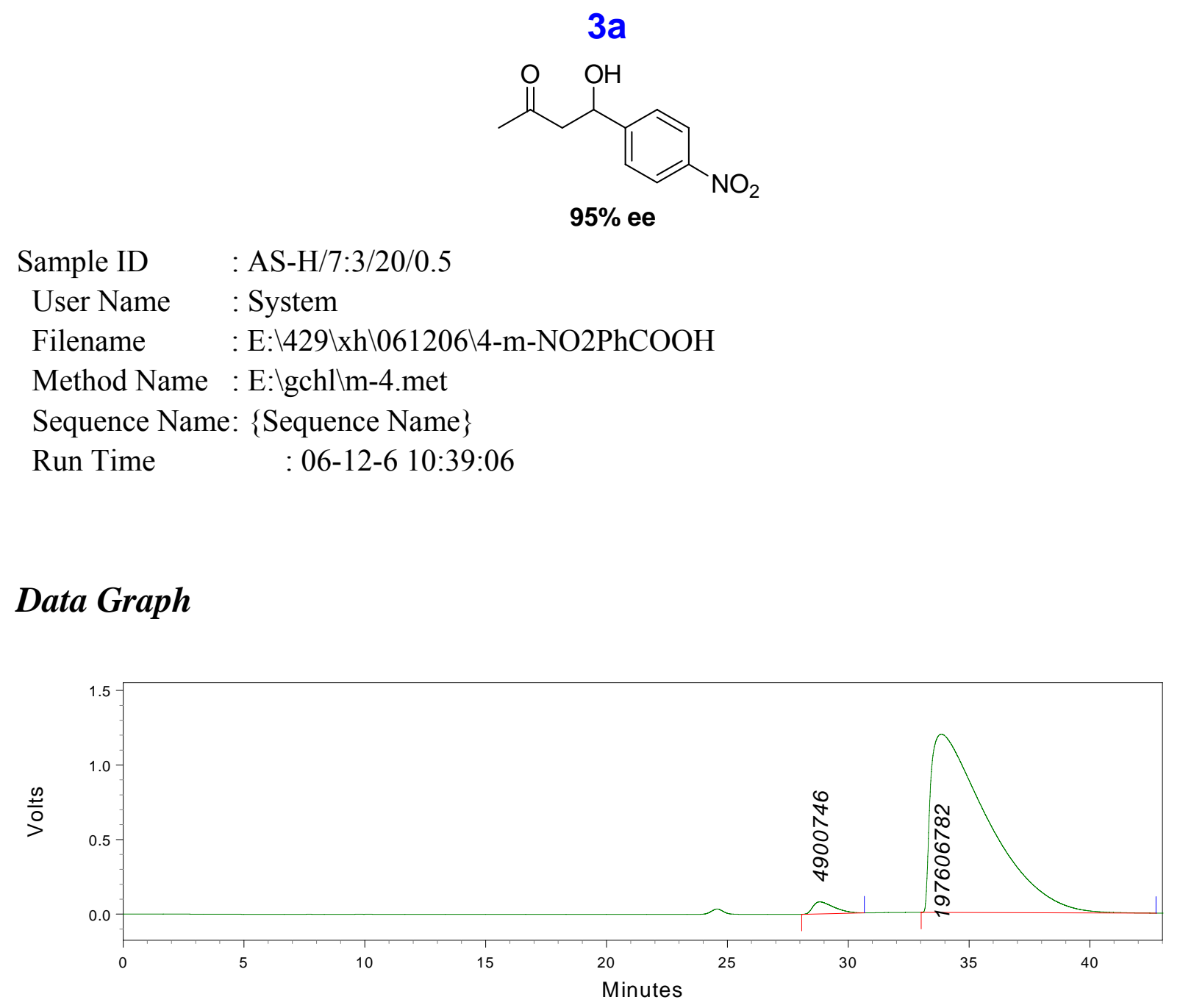

\section{Run Report}

\begin{tabular}{cccc}
$\begin{array}{c}\text { Detector A }(254 \mathrm{~nm}) \\
\text { Retention Time }\end{array}$ & Area & Area Percent \\
\hline 28.817 & 4900746 & 2.42 \\
33.852 & 197606782 & 97.58 \\
\hline Totals & & 100.00 \\
\hline
\end{tabular}




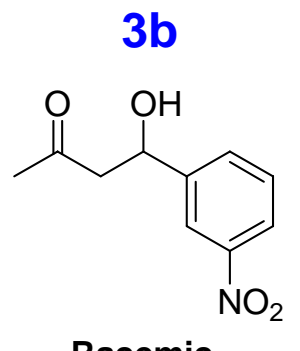

Racemic

Sample ID $\quad$ : OJ-H/1:4/25/0.8

User Name : System

Filename $\quad$ : E: $\backslash 429 \backslash \mathrm{xh} \backslash 061211 \backslash$ racemic-3-NO2-acetone-aldol

Method Name : E: Igchl $\backslash m-4$.met

Sequence Name: \{Sequence Name\}

Run Time : :06-12-11 19:39:28

\section{Data Graph}

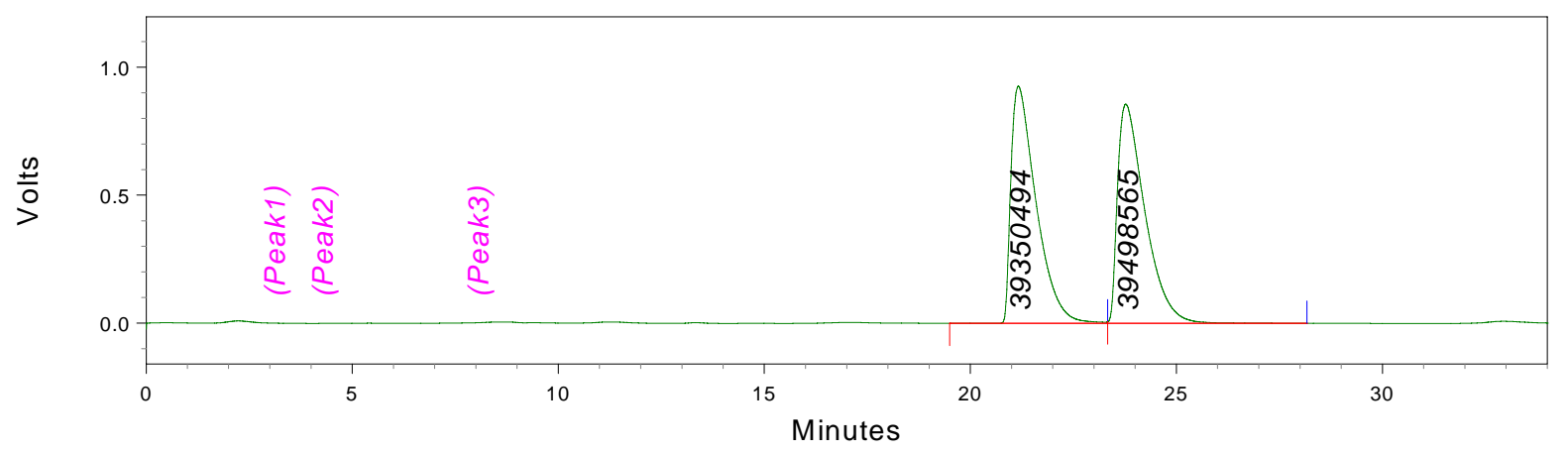

\section{Run Report}

Detector A $(254 \mathrm{~nm})$

Retention Time

Area

Area Percent

\begin{tabular}{|c|c|c|}
\hline 21.188 & 39350494 & 49.91 \\
23.798 & 39498565 & 50.09 \\
\hline Totals & & \\
& 78849058 & 100.00 \\
\hline
\end{tabular}




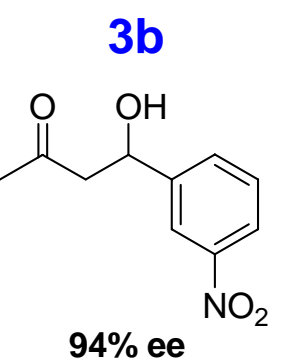
Sample ID $\quad:$ OJ-H/1:4/25/0.8
User Name : System
Filename : E: $4429 \backslash x h \backslash 061211 \backslash 4-3 \mathrm{NO} 2-3 \mathrm{NO} 2 \mathrm{PhCOOH}$
Method Name : E: $\mid g c h l \backslash m-4 . m e t$

Sequence Name: \{Sequence Name\}

Run Time $\quad$ :06-12-11 20:45:13

\section{Data Graph}

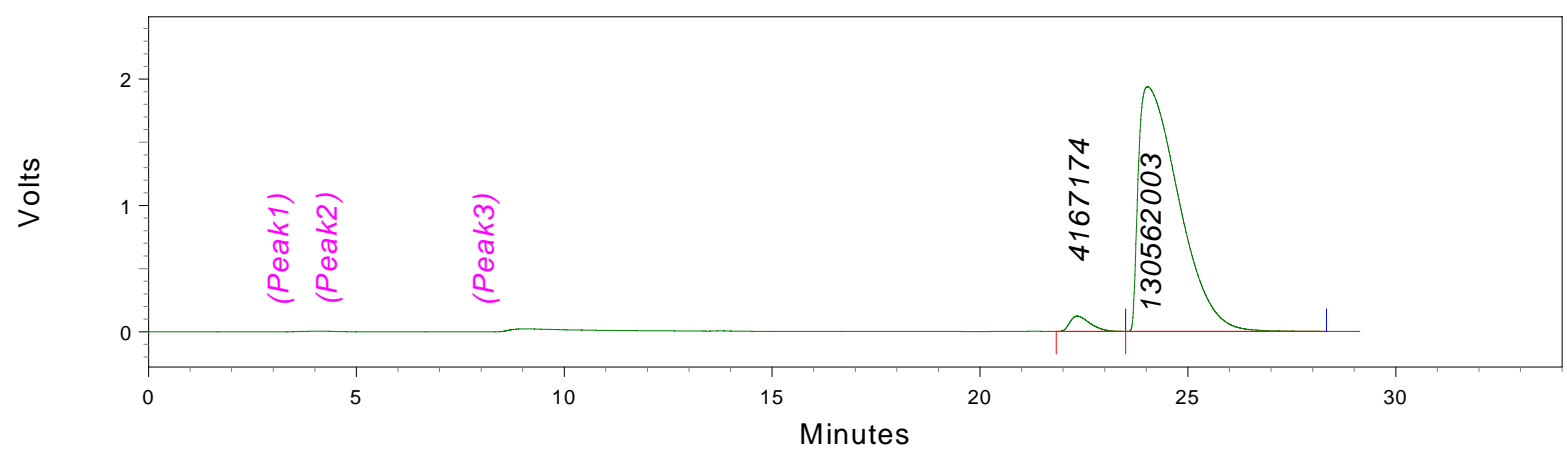

\section{Run Report}

Detector A $(254 \mathrm{~nm})$

Retention Time

Area

Area Percent

22.355

4167174

3.09

24.083

130562003

96.91

Totals 


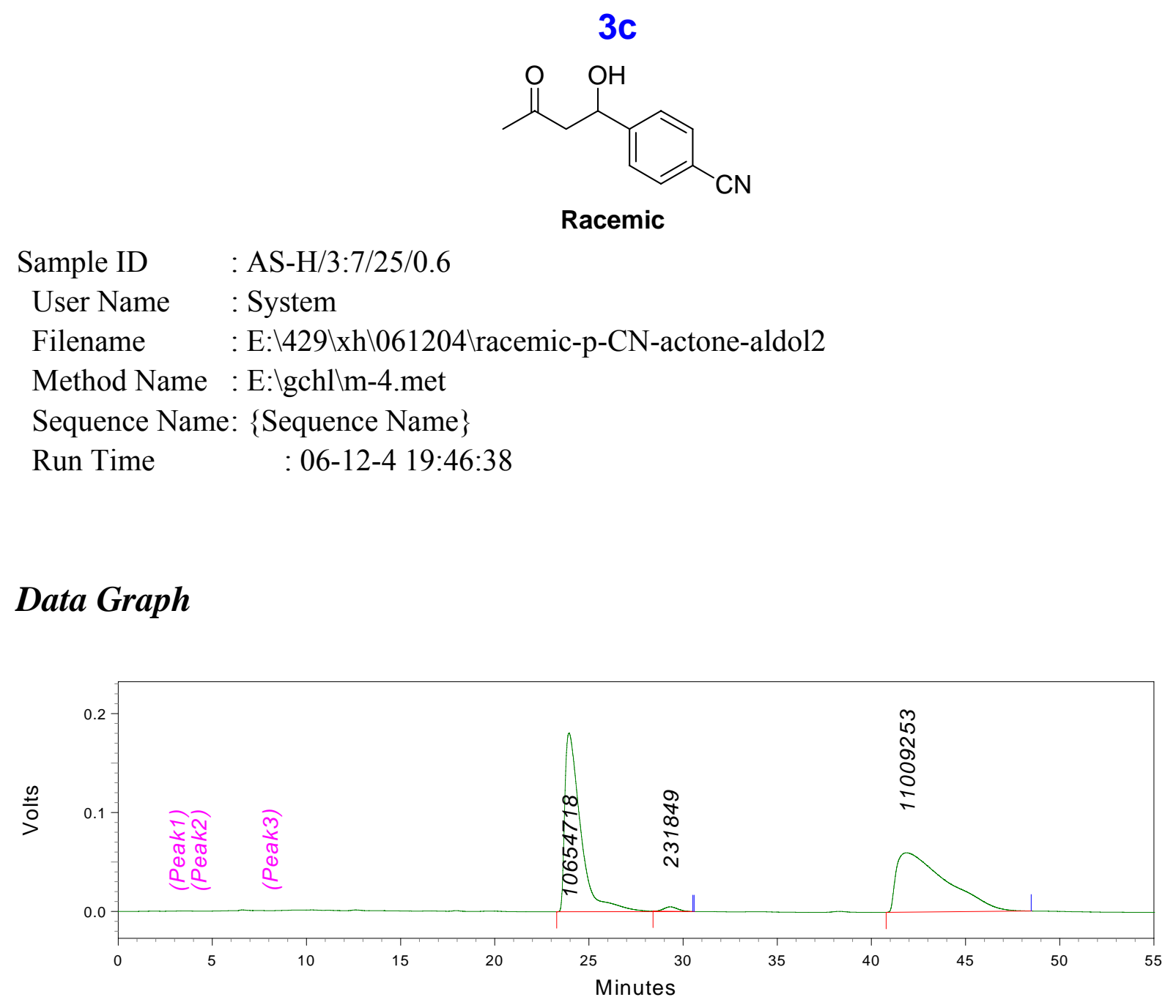

\section{Run Report}

Detector A (254nm)

Retention Time

Area

Area Percent

\begin{tabular}{|l|r|r|}
\hline 23.936 & 10654718 & 48.66 \\
29.318 & 231849 & 1.06 \\
41.865 & 11009253 & 50.28 \\
\hline Totals & 21895820 & 100.00 \\
\hline
\end{tabular}




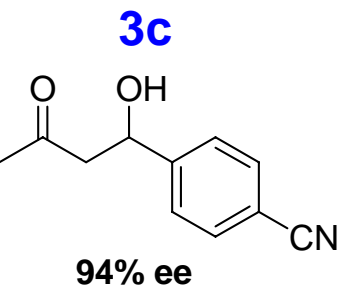

Sample ID $\quad:$ AS-H/3:7/25/0.5

User Name : System

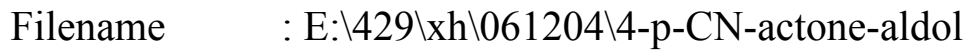

Method Name : E: $\mid g c h l \backslash m-4 . m e t$

Sequence Name: \{Sequence Name\}

Run Time : :06-12-4 17:06:30

\section{Data Graph}

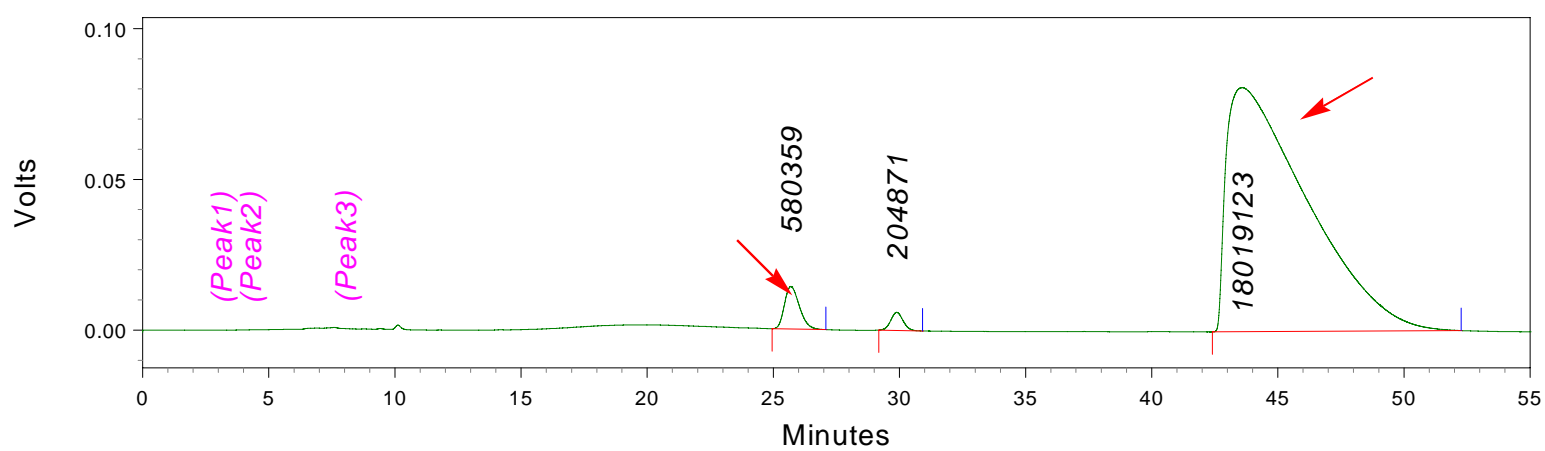

\section{Run Report}

\begin{tabular}{ccc}
$\begin{array}{c}\text { Detector A }(254 \mathrm{~nm}) \\
\text { Retention Time }\end{array}$ & Area & Area Percent \\
\hline 25.689 & 580359 & 3.09 \\
29.888 & 204871 & 1.09 \\
43.574 & 18019123 & 95.82 \\
\hline Totals & 18804353 & 100.00 \\
\hline
\end{tabular}




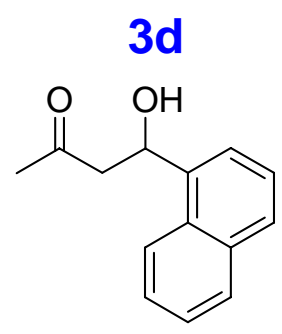

Racemic

$\begin{array}{ll}\text { Sample ID } & : \text { AS-H/15:85/25/0.8 } \\ \text { User Name } & : \text { System } \\ \text { Filename } & : \text { E: } \backslash 429 \backslash \mathrm{xh} \backslash 061211 \backslash \text { racemic-1-Nap-acetone-aldol } \\ \text { Method Name } & : \text { E: } \backslash \text { gchl } \backslash \mathrm{m}-4 . \text { met }\end{array}$

Sequence Name: \{Sequence Name\}

Run Time : :06-12-12 19:11:59

\section{Run}

Report

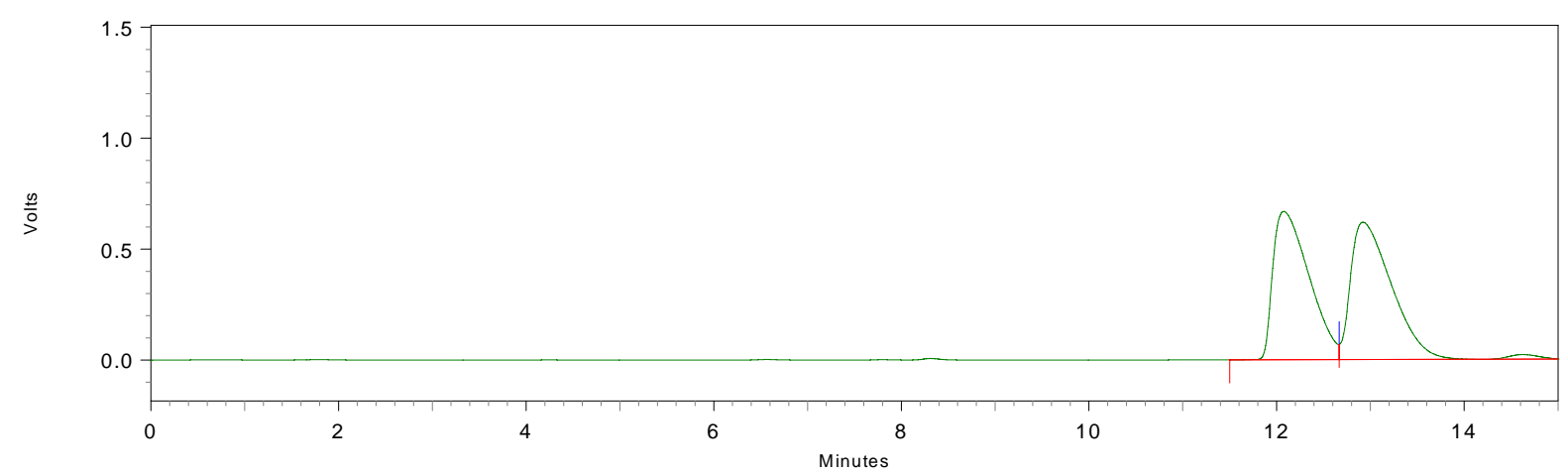

Detector A (254nm)

Retention Time

Area

Area Percent

\begin{tabular}{|c|c|c|}
\hline \multicolumn{1}{|c|}{35856969} & 48.95 \\
12.916 & 37399682 & 51.05 \\
\hline Totals & & \\
& 73256651 & 100.00 \\
\hline
\end{tabular}




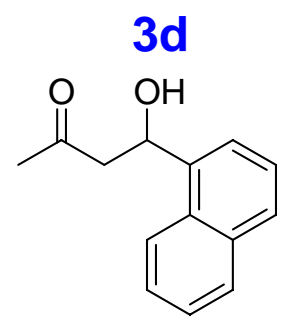

97\% ee
Sample ID
: AS-H/15:85/25/0.8
User Name : System
Filename $\quad:$ E: $\backslash 429 \backslash x h \backslash 061211 \backslash 18-1-N a p-m-N O 2 P h C O O H$
Method Name : E: Igchl $\backslash m-4$.met

Sequence Name: \{Sequence Name\}

Run Time : :06-12-12 19:29:14

\section{Run}

Report

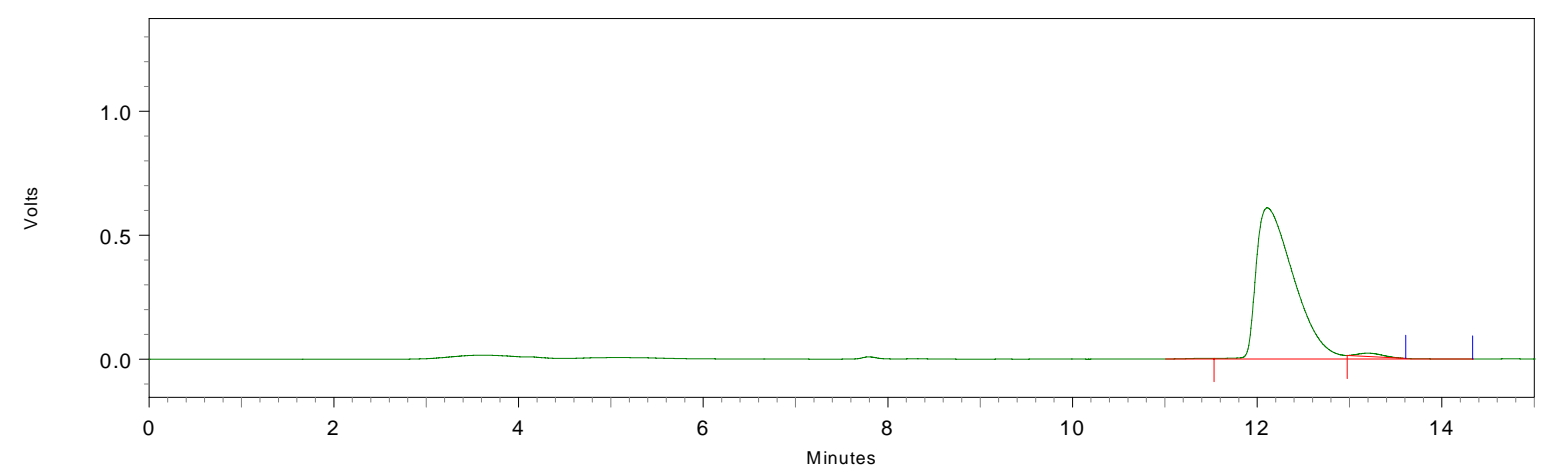

Detector A (254nm)

Retention Time

Area

Area Percent

12.105

35131073

98.64

13.192

483885

1.36

Totals 


\section{$3 e$}<smiles>CC(=O)CC(O)c1ccccc1Cl</smiles>

racemic

Sample ID $\quad$ : AS-H/0.6/25/254/1:9

User Name : System

Filename $\quad$ : E: $\backslash 429 \backslash \mathrm{xh} \backslash 061229 \backslash$ racemic-acetone-o-Cl

Method Name : E:\wzj\11.met

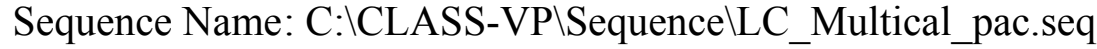

Run Time

: 06-12-29 20:14:55

\section{Data Graph}

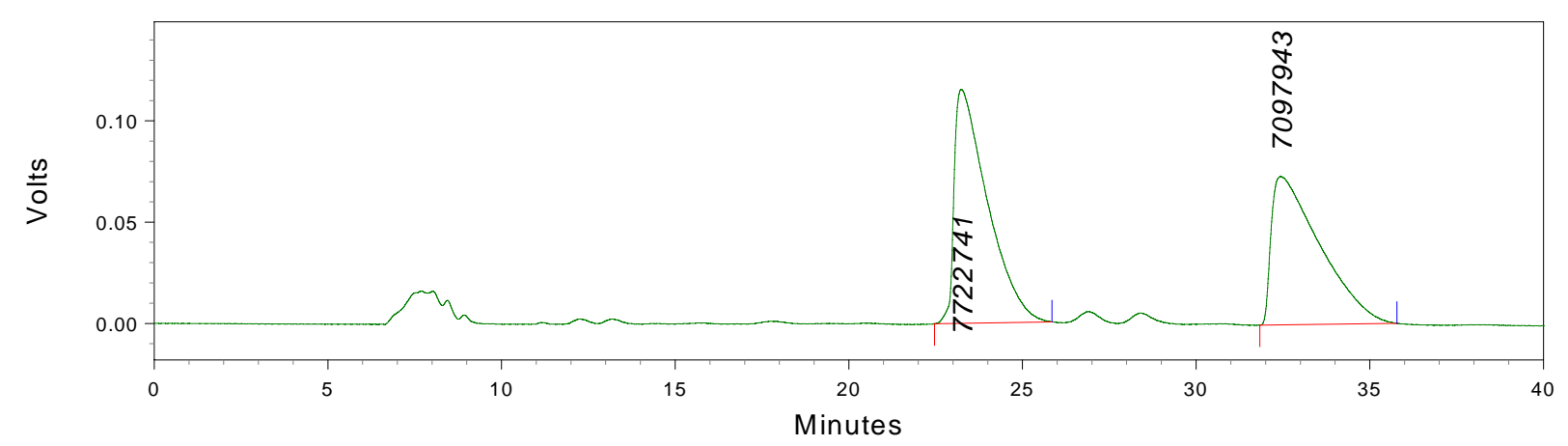

\section{Run Report}

Detector A (254nm)

\begin{tabular}{|ccc|} 
Retention Time & Area & Area Percent \\
\hline 23.235 & 7722741 & 52.11 \\
32.432 & 7097943 & 47.89 \\
\hline Totals & 14820684 & 100.00 \\
\hline
\end{tabular}




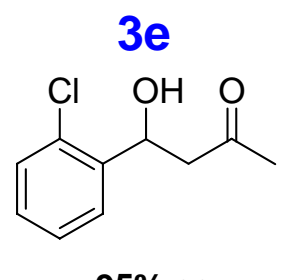

Sample ID $\quad$ : AS-H/0.6/25/254/1:9

User Name : System

Filename $\quad: \mathrm{E}: \backslash 429 \backslash \mathrm{xh} \backslash 061229 \backslash$ acetone-o-Cl-mNO2PhCOOH

Method Name : E:\wzj\11.met

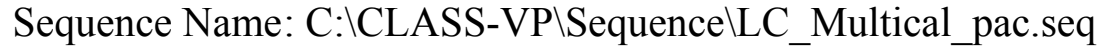

Run Time : :06-12-29 20:57:04

\section{Data Graph}

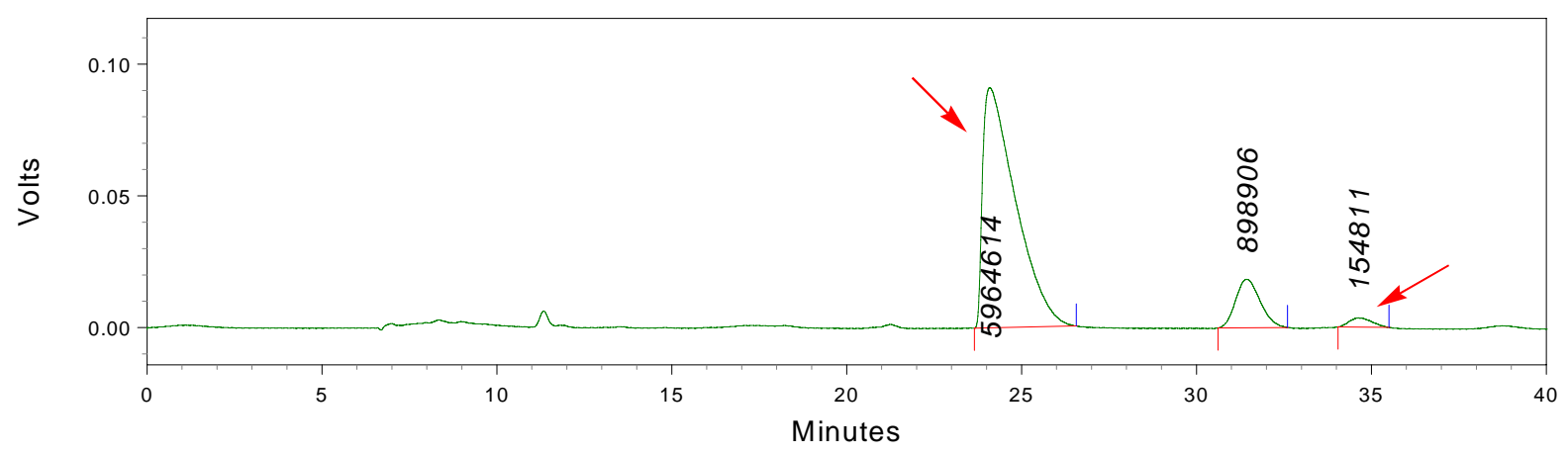

\section{Run Report}

\begin{tabular}{c|cc|}
$\begin{array}{c}\text { Detector A }(254 \mathrm{~nm}) \\
\text { Retention Time }\end{array}$ & Area & Area Percent \\
\hline 24.087 & 5964614 & 84.99 \\
31.422 & 898906 & 12.81 \\
34.623 & 154811 & 2.21 \\
\hline Totals & & 100.00 \\
\hline
\end{tabular}


<smiles>CC(=O)CC(O)c1cccc(Br)c1</smiles>

Racemic

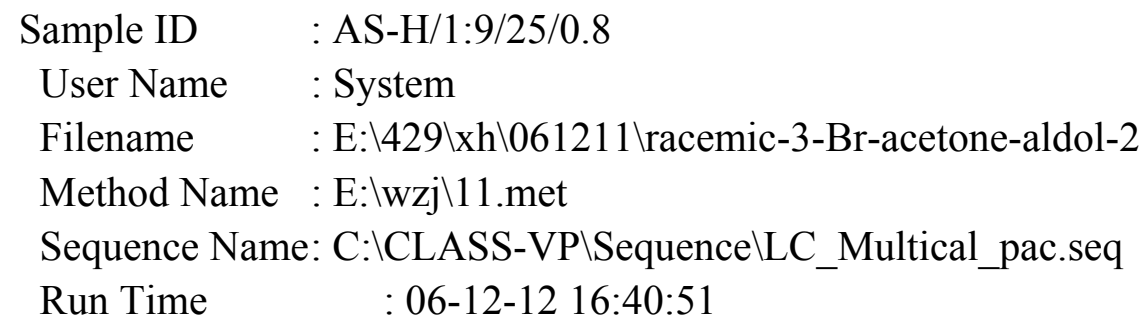

\section{Run}

\section{Report}

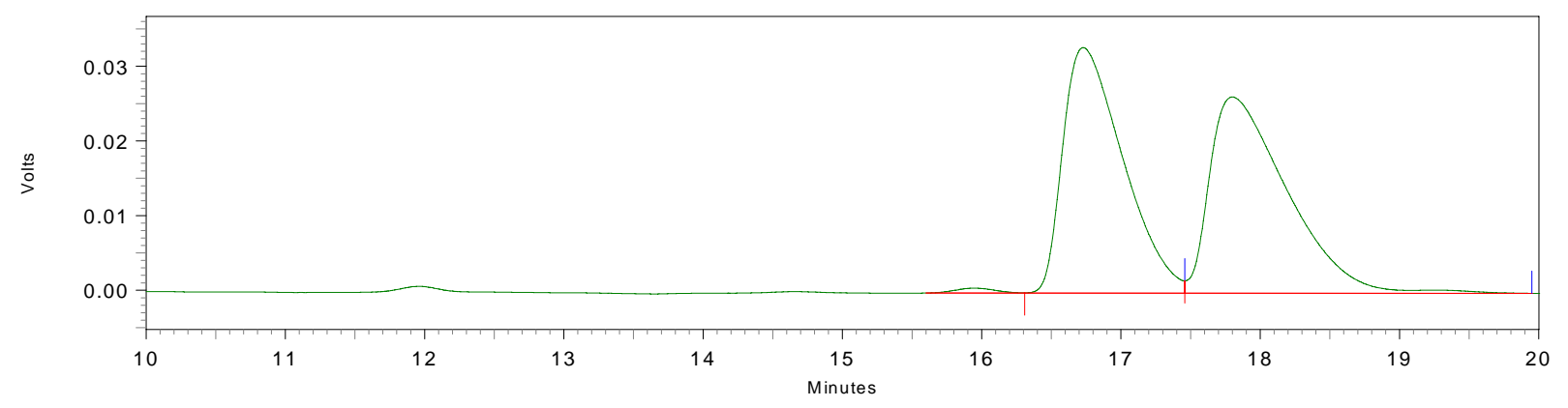

Detector A (254nm)

\begin{tabular}{|ccc|} 
Retention Time & Area & Area Percent \\
\hline 16.724 & 989831 & 49.42 \\
17.796 & 1012884 & 50.58 \\
\hline Totals & 2002716 & 100.00 \\
\hline
\end{tabular}




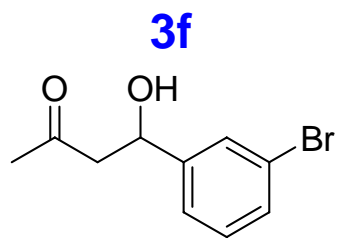

$92 \%$ ee

Sample ID $\quad$ : AS-H/1:9/25/0.8

User Name : System

Filename $\quad$ : E: $\backslash 429 \backslash \mathrm{xh} \backslash 061211 \backslash 16-3-\mathrm{Br}-\mathrm{m}-\mathrm{NO} 2 \mathrm{PhCOOH}$

Method Name : E: \wzj\11.met

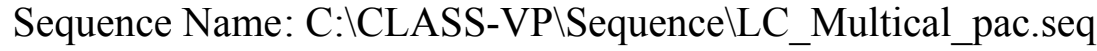

Run Time : :06-12-12 15:52:11

\section{Run}

\section{Report}

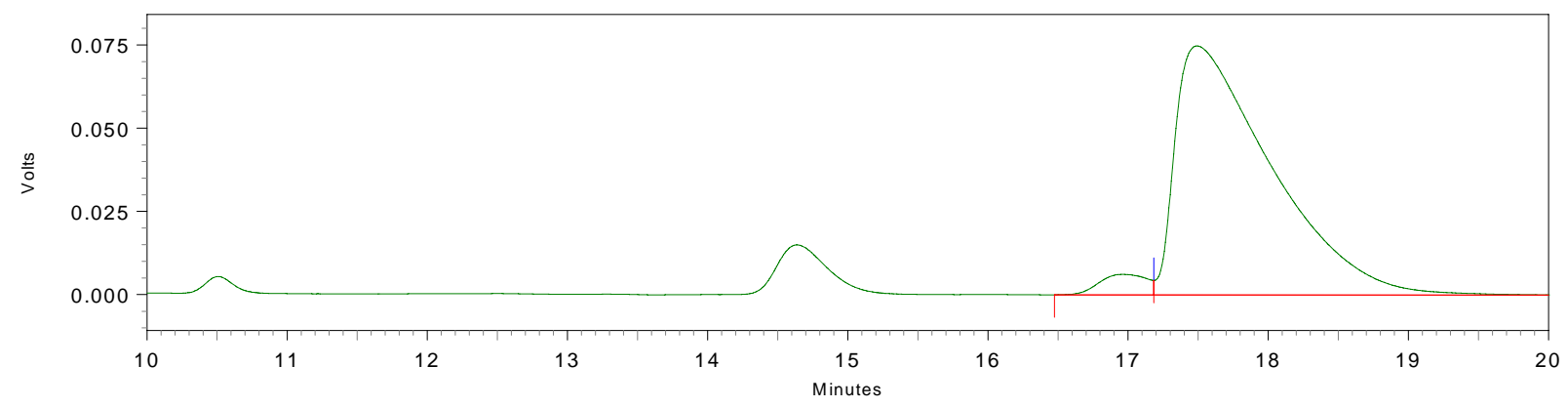

Detector A (254nm)

Retention Time

Area

Area Percent

\begin{tabular}{|c|c|c|}
\hline \multicolumn{1}{|c|}{16.956} & 145317 & 3.92 \\
17.488 & 3561126 & 96.08 \\
\hline Totals & & 100.00 \\
\hline
\end{tabular}


$3 g$<smiles>CC(=O)CC(O)c1ccccc1</smiles>

Racemic
Sample ID $\quad$ : AS-H/15:85/25/0.8
User Name : System
Filename $\quad$ : E: $\backslash 429 \backslash \mathrm{xh} \backslash 061211 \backslash$ racemic-4-H-acetone-aldol
Method Name : E: \wzj\11.met

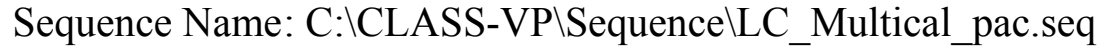
Run Time : :06-12-12 18:37:32

\section{Run}

\section{Report}

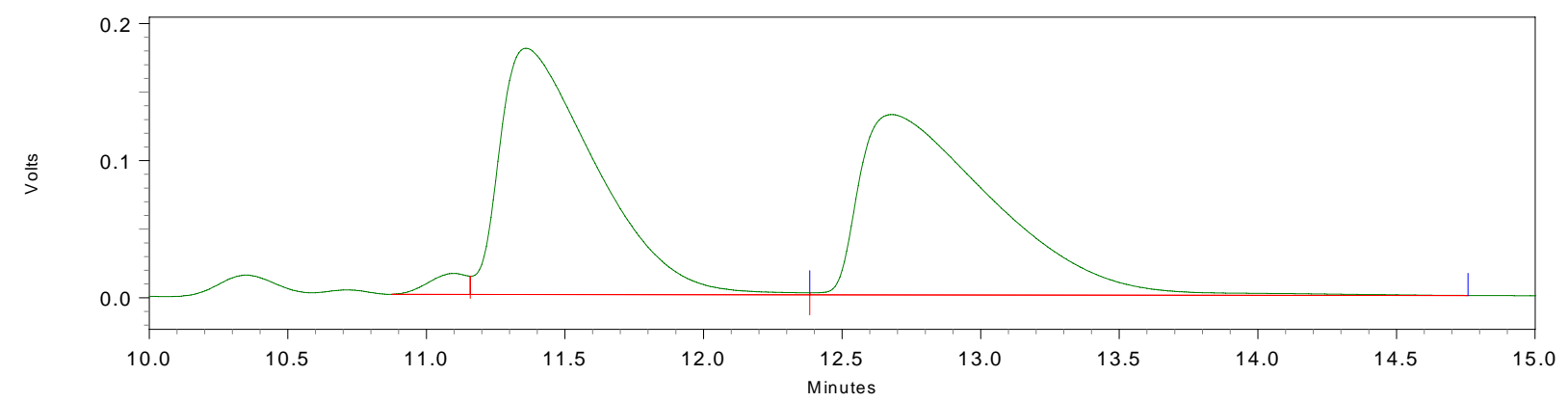

Detector A (254nm)

Retention Time Area

Area Percent

\begin{tabular}{|c|c|c|}
\multicolumn{1}{c}{ Retention Time } & Area & Area Percent \\
\hline 11.355 & 4310419 & 49.56 \\
12.674 & 4387555 & 50.44 \\
\hline Totals & & 100.00 \\
\hline
\end{tabular}


<smiles>CC(=O)CC(O)c1ccccc1</smiles>

$94 \%$ ee

Sample ID : AS-H/15:85/25/0.8

User Name : System

Filename $\quad$ : E: $\backslash 429 \backslash \mathrm{xh} \backslash 061211 \backslash 17-4-\mathrm{H} \mathrm{m}-\mathrm{NO} 2 \mathrm{PhCOOH}$

Method Name : E: \wzj\11.met

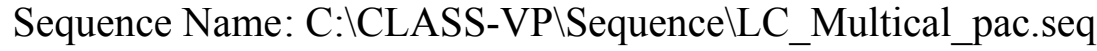

Run Time : :06-12-12 18:55:08

\section{Run}

\section{Report}

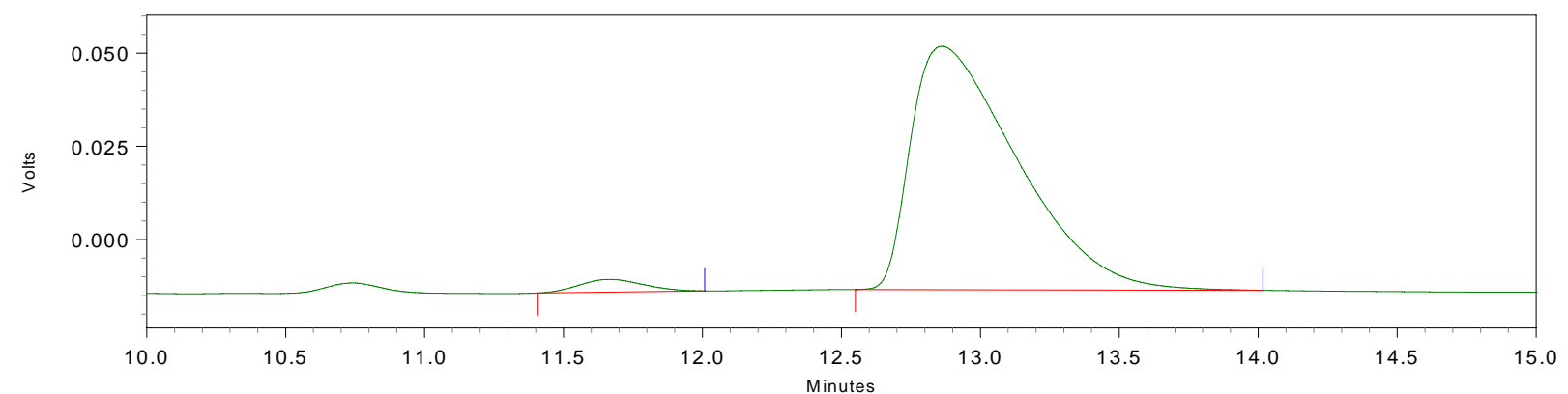

Detector A (254nm)

Retention Time Area Area Percent 11.661 54833 3.06

12.857

1738465

96.94

\section{Totals}




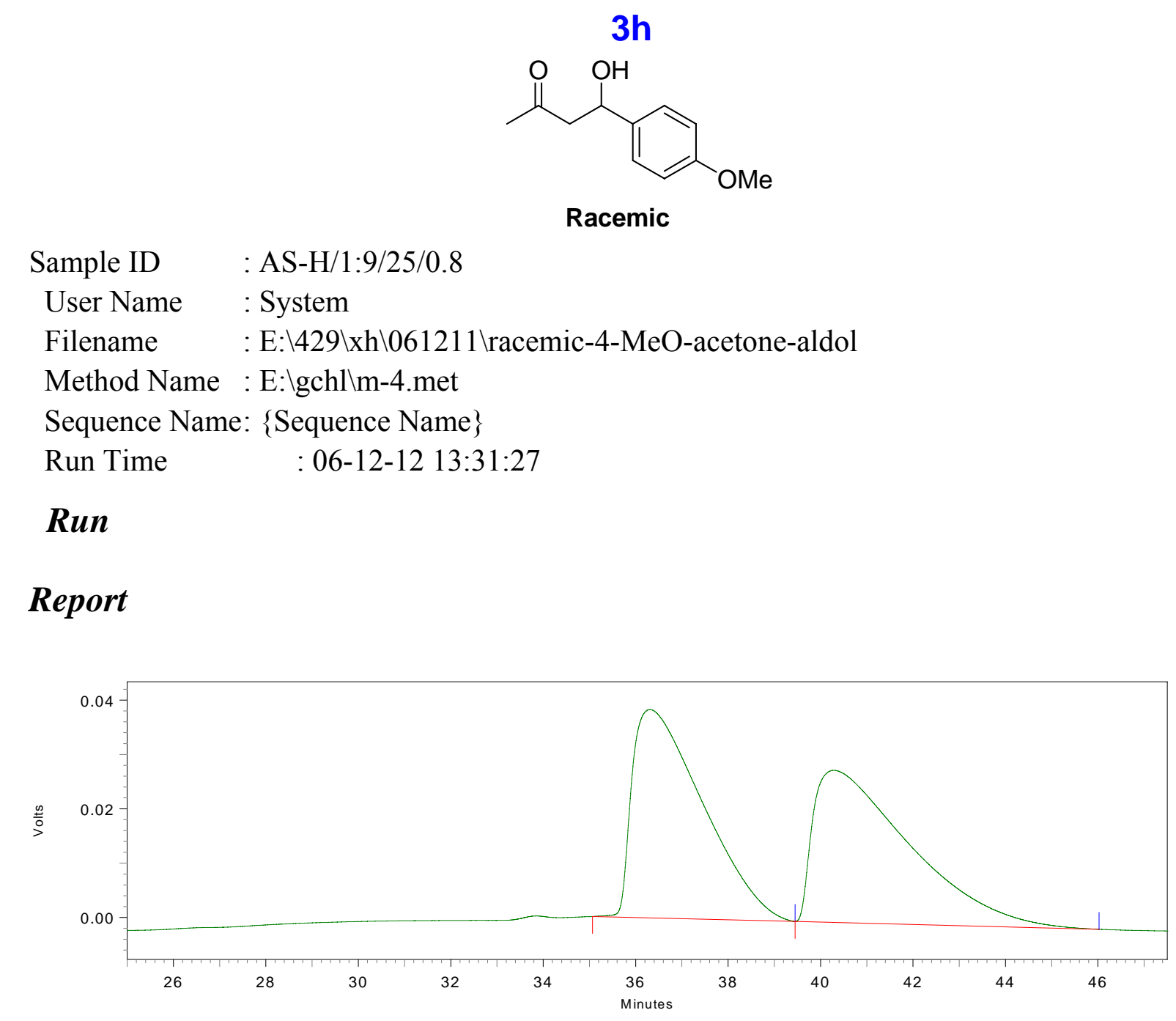

\begin{tabular}{c|c|c|}
$\begin{array}{c}\text { Detector A }(254 \mathrm{~nm}) \\
\text { Retention Time }\end{array}$ & Area & Area Percent \\
\hline 36.304 & 4066058 & 50.15 \\
40.284 & 4041858 & 49.85 \\
\hline Totals & & 100.00 \\
\hline
\end{tabular}




\section{3h}<smiles>COc1ccc(C(O)CC(C)=O)cc1</smiles>

$93 \%$ ee

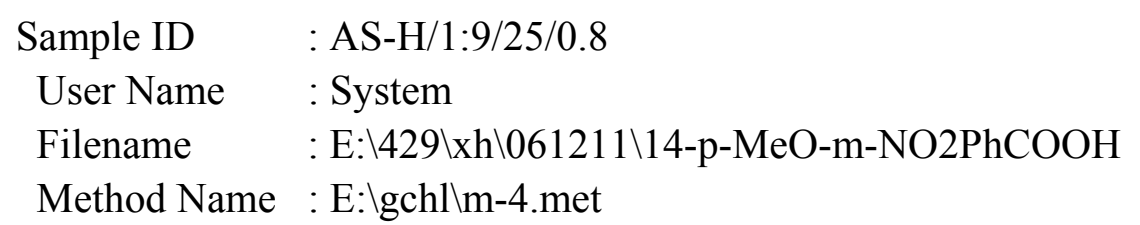

Sequence Name: \{Sequence Name\}

Run Time $\quad$ :06-12-12 14:25:09

\section{Run}

\section{Report}

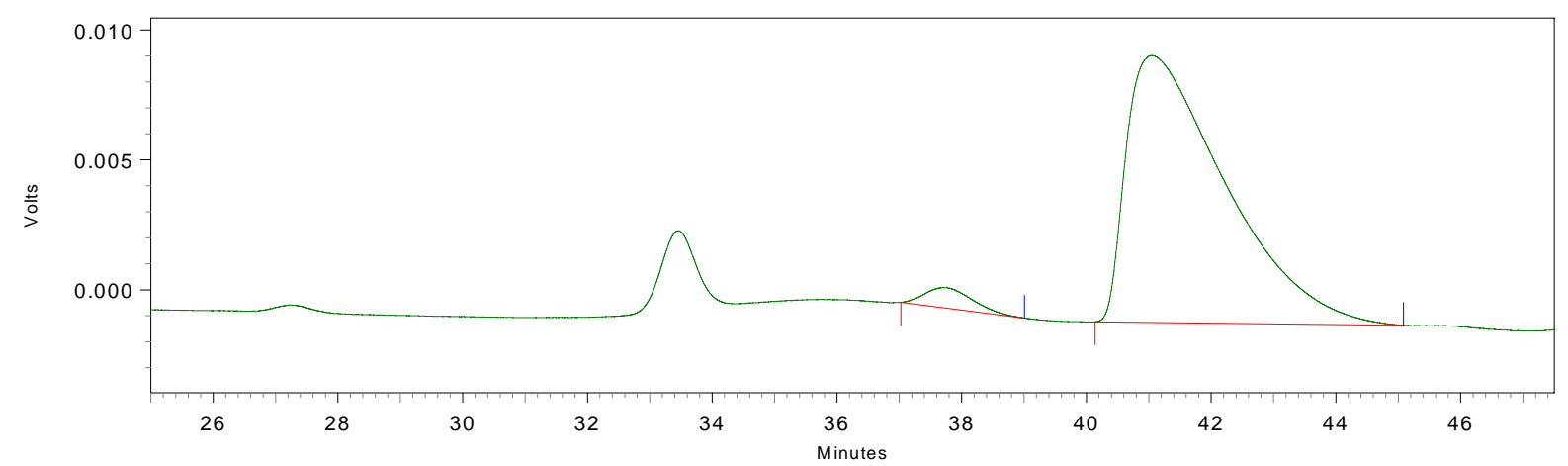

Detector A (254nm)

Retention Time

Area

Area Percent

\begin{tabular}{|ccc|}
\multicolumn{1}{c}{ Area } & Area Percent \\
\hline Retention Time & 41529 & 3.53 \\
47.701 & 1133667 & 96.47 \\
\hline Totals & 1175196 & 100.00 \\
\hline
\end{tabular}




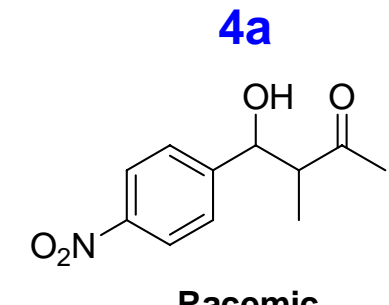

\section{Racemic}

Sample ID $\quad$ : AS-H/3:7/25/0.5/254

User Name : System

Filename $\quad:$ E: $4429 \backslash x h \backslash 061217 \backslash$ racemic-butone-p-NO2-aldol

Method Name : E:Iwzj\11.met

Sequence Name: C:ICLASS-VPISequencelLC_Multical_pac.seq

Run Time : :06-12-17 11:32:30

\section{Run}

\section{Report}

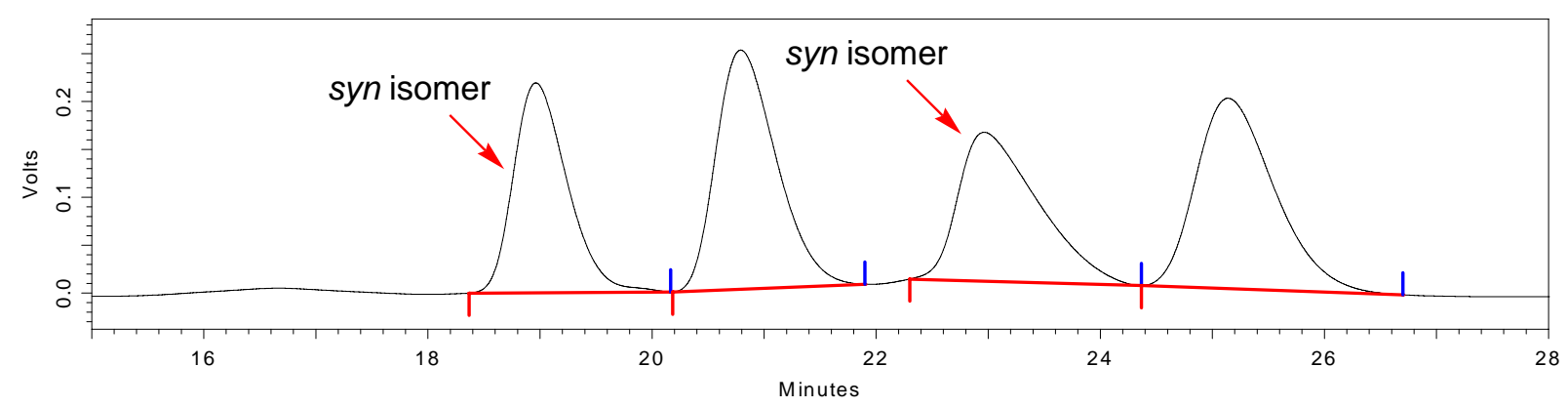

Detector A (254nm)

Retention Time

Area

Area Percent 


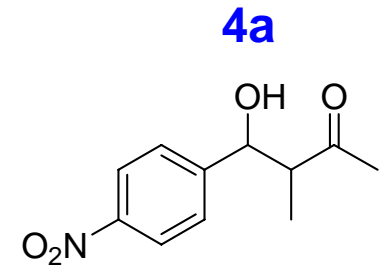

for syn isomer: $96 \%$ ee
Sample ID : AS-H/3:7/25/0.5/254
User Name : System
Filename $\quad:$ E: $\backslash 429 \backslash x h \backslash 061217 \backslash 3$-butone-p-NO2-aldol-cool
Method Name : E: $\mid w z j \backslash 11 . m e t$
Sequence Name: C:\CLASS-VP \SequencelLC_Multical_pac.seq
Run Time : :06-12-17 12:02:03

\section{Run}

\section{Report}

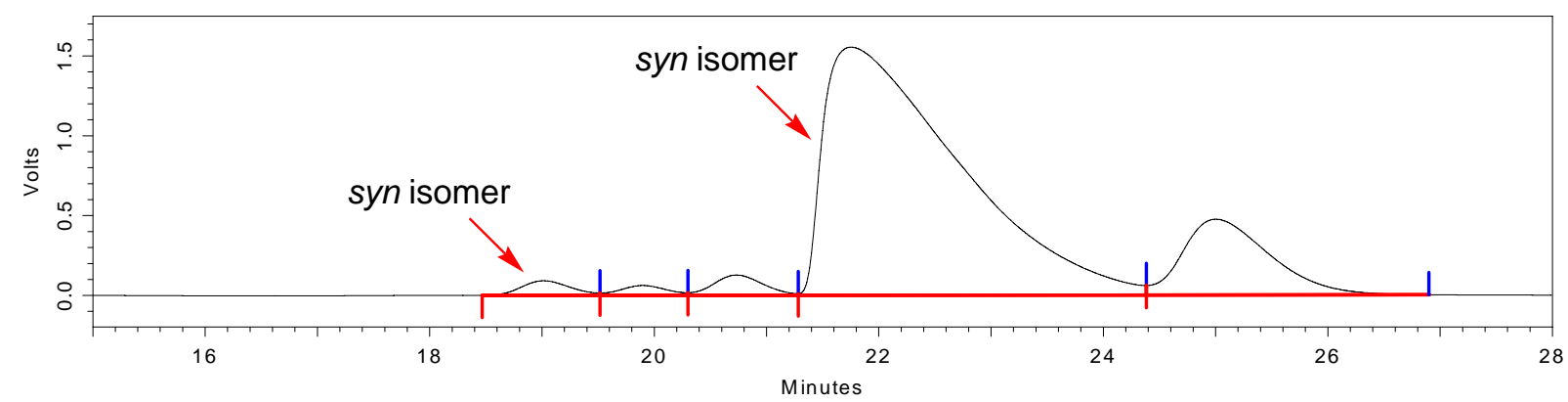

Detector A (254nm)

\begin{tabular}{ccc} 
Retention Time & Area & Area Percent \\
\hline 19.009 & 2724633 & 1.66 \\
19.894 & 1727267 & 1.05 \\
20.727 & 3814778 & 2.33 \\
21.750 & 130223504 & 79.42 \\
24.998 & 25481892 & 15.54 \\
\hline Totals & 163972074 & 100.00 \\
\hline
\end{tabular}




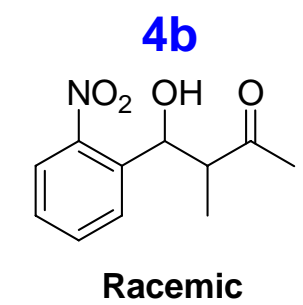
Sample ID $\quad$ : AS-H/3:7/25/0.6/254
User Name : System
Filename $\quad:$ E: $429 \backslash \times h \backslash 061217 \backslash$ racemic-butone-o-NO2-aldol
Method Name : E: $\mid g c h l \backslash m-4 . m e t$

Sequence Name: \{Sequence Name\}

Run Time : :06-12-17 12:43:24

\section{Run}

\section{Report}

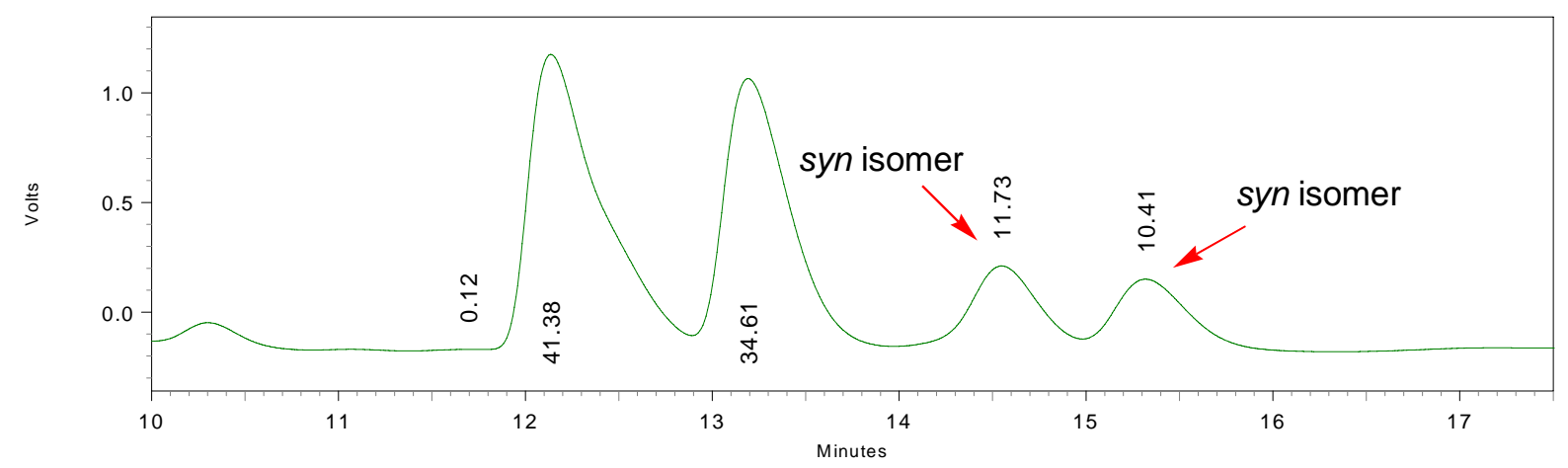

Detector A (254nm)

\begin{tabular}{|ccc|}
\hline Retention Time & Area & Area Percent \\
\hline 11.688 & 107970 & 0.12 \\
12.132 & 36585461 & 41.38 \\
13.188 & 30598253 & 34.61 \\
14.544 & 10369683 & 11.73 \\
15.314 & 9206119 & 10.41 \\
18.125 & 1315176 & 1.49 \\
20.290 & 229666 & 0.26 \\
\hline Totals & & 100.00 \\
\hline
\end{tabular}




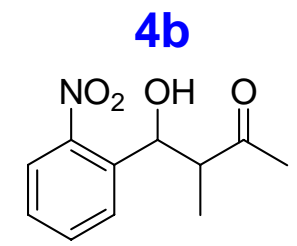

for syn isomer: $96 \%$ ee

Sample ID : AS-H/3:7/25/0.6/254

User Name : System

Filename $\quad$ : E: $4429 \backslash x h \backslash 061217 \backslash 4$-butone-o-NO2-aldol

Method Name : E:lgchl $\backslash$ m-4.met

Sequence Name: \{Sequence Name\}

Run Time : :06-12-17 13:05:01

\section{Run}

\section{Report}

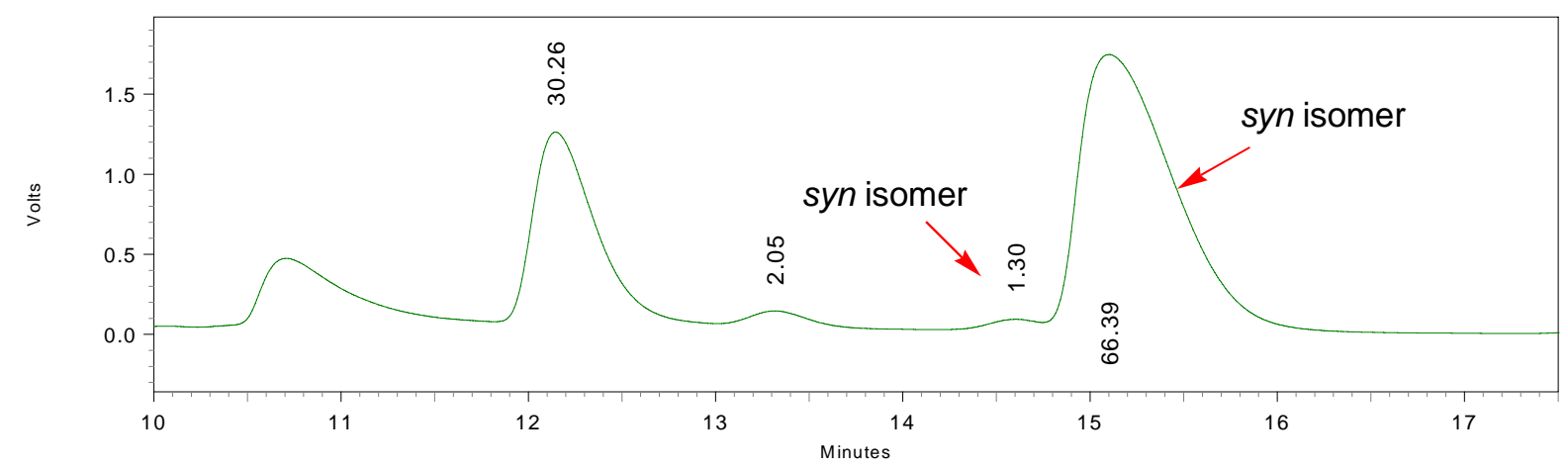

Detector A (254nm)

Retention Time

\begin{tabular}{|ccc|}
\hline 12.141 & Area & Area Percent \\
13.312 & 27088272 & 30.26 \\
14.598 & 1834701 & 2.05 \\
15.097 & 1166060 & 1.30 \\
\hline Totals & 59430295 & 66.39 \\
\hline
\end{tabular}




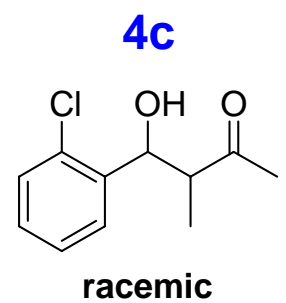

Sample ID $\quad$ : AS-H/0.5/25/254/1:9

User Name : System

Filename $\quad:$ E: $\backslash 429 \backslash x h \backslash 061229 \backslash$ racemic-butone-o-Cl-again-2

Method Name : E:lgchl \m-4.met

Sequence Name: \{Sequence Name\}

Run Time : :06-12-29 18:24:04

\section{Data Graph}

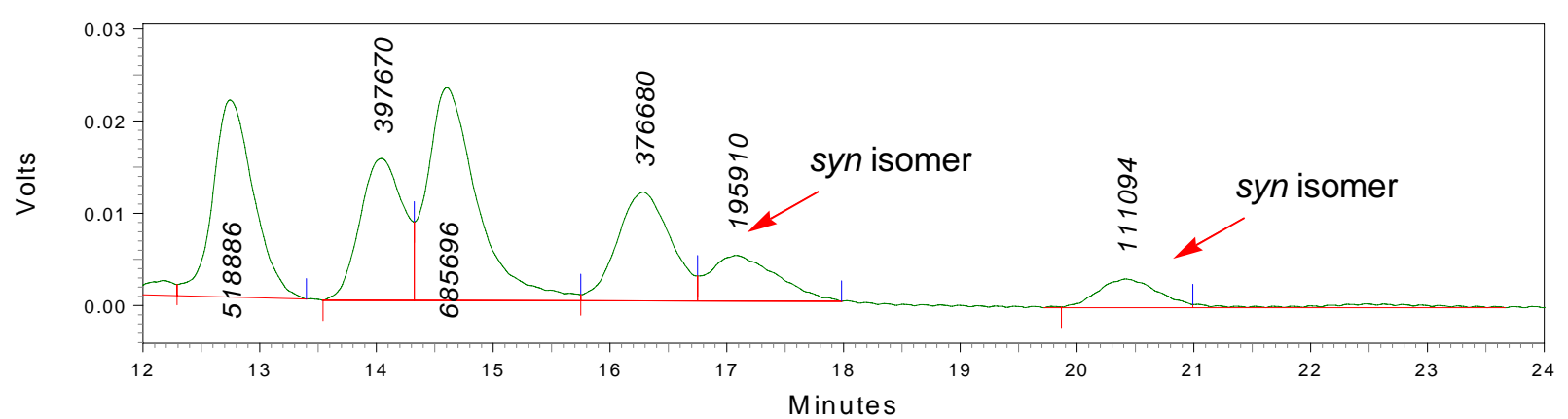

\section{Run Report}

Detector A (254nm)

\begin{tabular}{ccc} 
Retention Time & Area & Area Percent \\
\hline 12.742 & 518886 & 22.70 \\
14.038 & 397670 & 17.40 \\
14.600 & 685696 & 30.00 \\
16.280 & 376680 & 16.48 \\
17.078 & 195910 & 8.57 \\
20.410 & 111094 & 4.86 \\
\hline Totals & & 100.00 \\
\hline
\end{tabular}




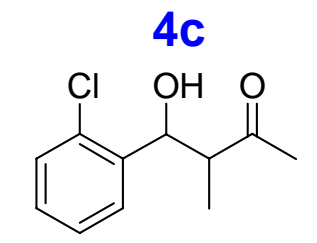

\section{for syn isomer $87 \%$ ee}

Sample ID : : AS-H/1:9/0.6/25/254

User Name : System

Filename $\quad:$ E: $\backslash 429 \backslash x h \backslash 061227 \backslash$ butone-2-Cl-syn-again

Method Name : E: $\backslash g c h l \backslash m-4 . m e t$

Sequence Name: \{Sequence Name\}

Run Time : :06-12-27 23:03:38

\section{Data Graph}

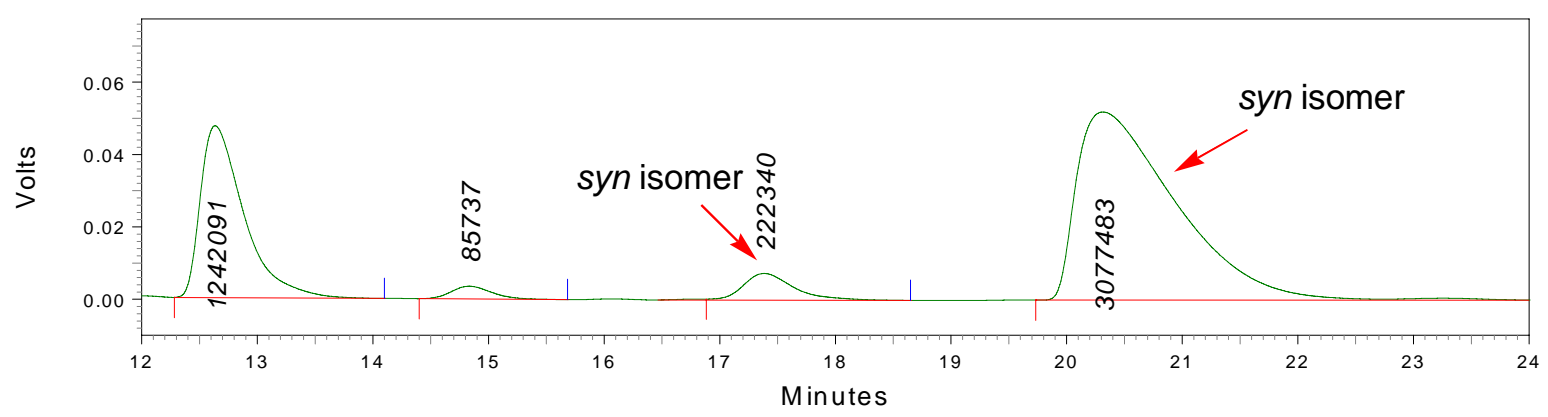

\section{Run Report}

Detector A (254nm)

\begin{tabular}{|ccc|}
\hline Retention Time & Area & Area Percent \\
\hline 12.631 & 1242091 & 26.84 \\
14.829 & 85737 & 1.85 \\
17.379 & 222340 & 4.80 \\
20.310 & 3077483 & 66.50 \\
\hline Totals & & 100.00 \\
\hline
\end{tabular}


<smiles>CC(=O)C(C)C(O)c1cccc2ccccc12</smiles>

Racemic

Printed : 2007-2-8 12:19:38

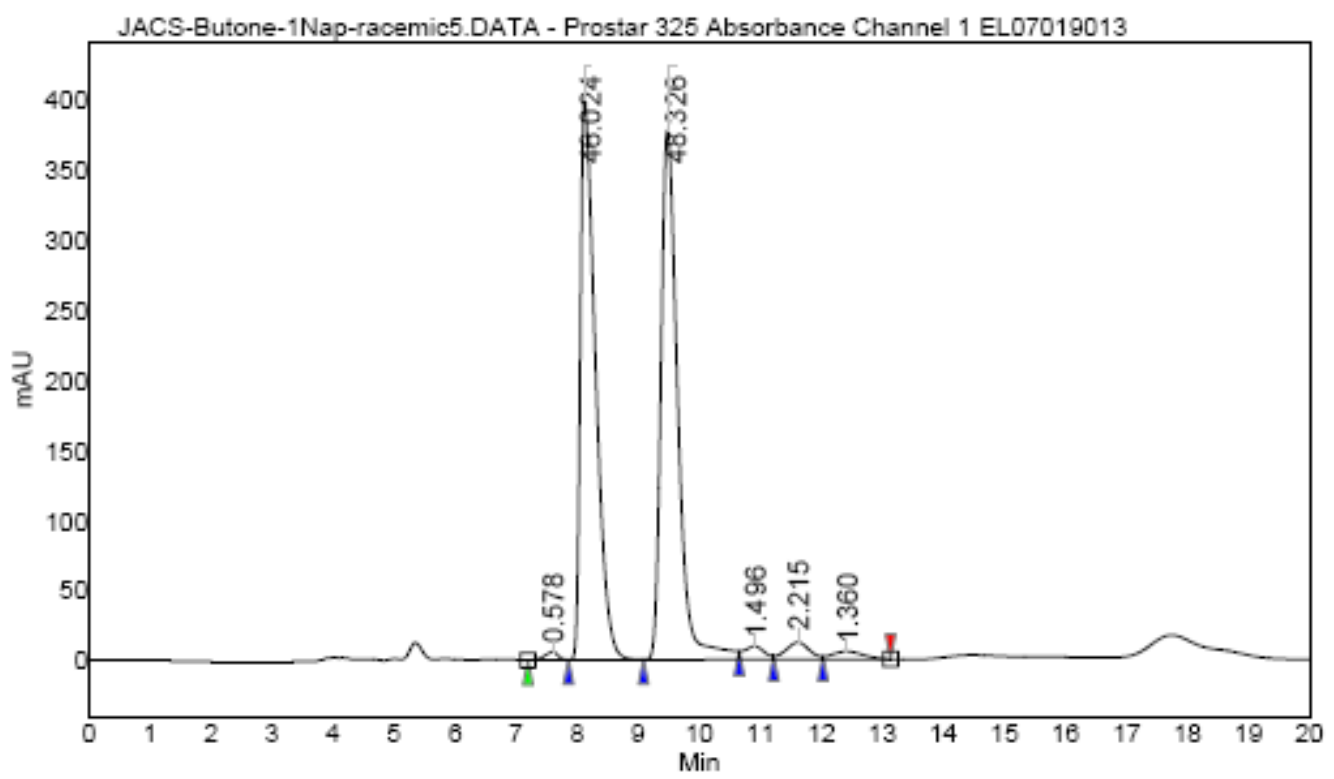

Peak results :

\begin{tabular}{|c|l|r|r|r|r|r|}
\hline Index & Name & $\begin{array}{r}\text { Time } \\
\text { [Min] }\end{array}$ & $\begin{array}{r}\text { Quantity } \\
\text { [\% Area] }\end{array}$ & $\begin{array}{r}\text { Height } \\
\text { [mAU] }\end{array}$ & $\begin{array}{r}\text { Area } \\
\text { [mAU.Min] }\end{array}$ & $\begin{array}{r}\text { Area \% } \\
\text { [\%] }\end{array}$ \\
\hline \hline 1 & UNKNOWN & 7.60 & 0.58 & 6.0 & 1.4 & 0.578 \\
\hline 2 & UNKNOWN & 8.12 & 46.02 & 400.2 & 114.8 & 46.024 \\
\hline 3 & UNKNOWN & 9.48 & 48.33 & 377.6 & 120.5 & 48.326 \\
\hline 4 & UNKNOWN & 10.90 & 1.50 & 9.5 & 3.7 & 1.496 \\
\hline 5 & UNKNOWN & 11.62 & 2.22 & 12.3 & 5.5 & 2.215 \\
\hline 6 & UNKNOWN & 12.41 & 1.36 & 5.7 & 3.4 & 1.360 \\
\hline & & & & & & \\
\hline Total & & & 100.00 & 811.2 & 249.4 & 100.000 \\
\hline
\end{tabular}




\section{ICCAS}

Chromatogram : JACS-Butone-1Nap-sample5_channel1

System : HPLC

Method : $\mathrm{XH}-1-4$

User : XH

Acquired : 2007-2-7 11:46:47

Processed : 2007-2-7 12:15:16

Printed : 2007-2-8 12:17:02

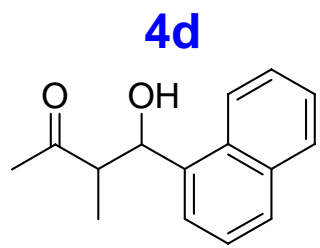

for syn isomer $92 \%$ ee

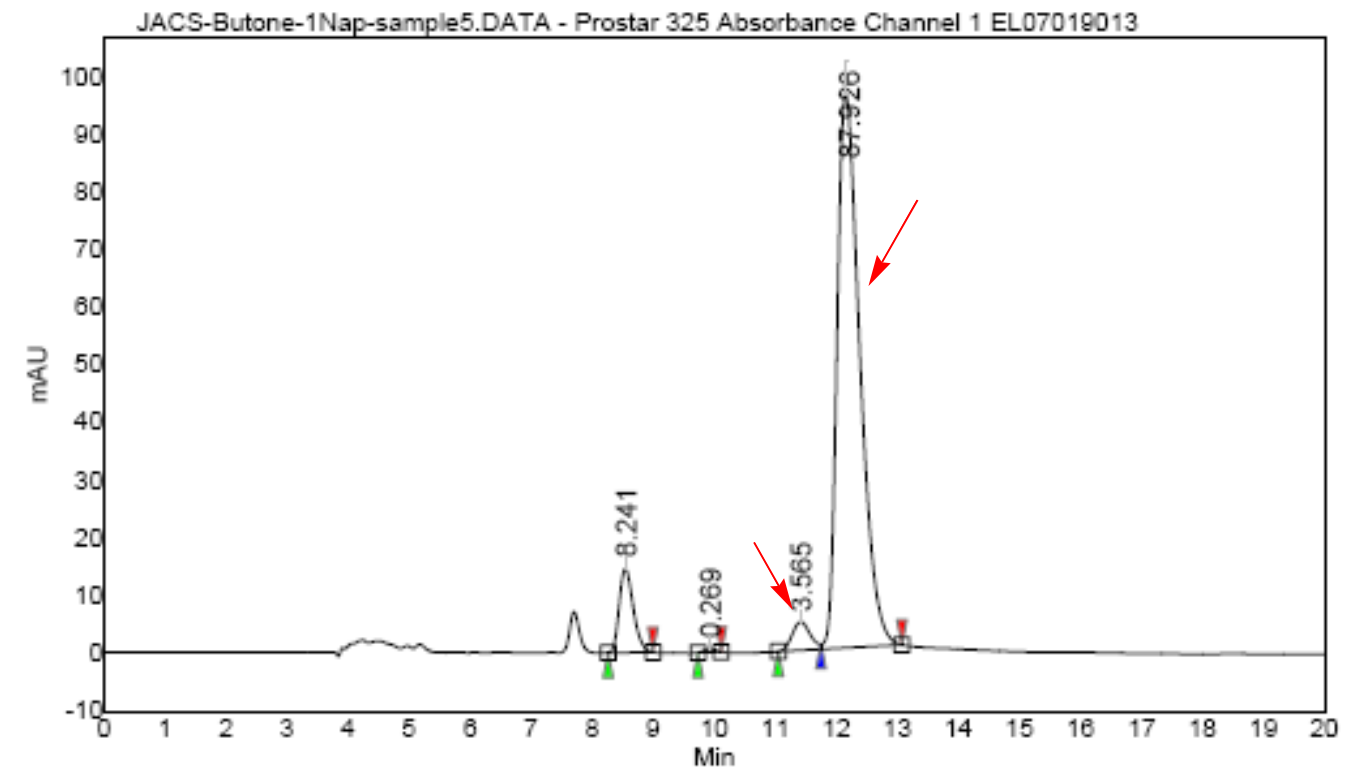

Peak results :

\begin{tabular}{|c|l|r|r|r|r|r|}
\hline Index & Name & $\begin{array}{r}\text { Time } \\
\text { [Min] }]\end{array}$ & $\begin{array}{r}\text { Quantity } \\
\text { [\% Area] }\end{array}$ & $\begin{array}{r}\text { Height } \\
\text { [mAU] }\end{array}$ & $\begin{array}{r}\text { Area } \\
\text { [mAU.Min] }\end{array}$ & $\begin{array}{r}\text { Area \% } \\
{[\%]}\end{array}$ \\
\hline \hline 1 & UNKNOWN & 8.54 & 8.24 & 14.6 & 3.9 & 8.241 \\
\hline 2 & UNKNOWN & 9.93 & 0.27 & 0.6 & 0.1 & 0.269 \\
\hline 3 & UNKNOWN & 11.41 & 3.56 & 4.9 & 1.7 & 3.565 \\
\hline 4 & UNKNOWN & 12.15 & 87.93 & 96.1 & 41.4 & 87.926 \\
\hline & & & & & & \\
\hline Total & & & 100.00 & 116.2 & 47.1 & 100.000 \\
\hline
\end{tabular}




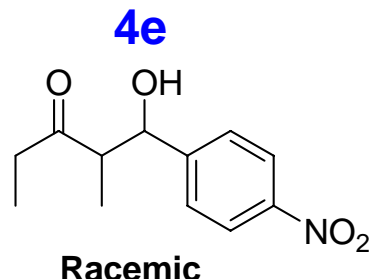

Sample ID : As-H/0.8/20/254/5:95

User Name : System

Filename : E: $4429 \backslash x h \backslash 061230 \backslash$ racemic-3wutong-ASH-1

Method Name : E: $\mid g c h l \backslash m-4 . m e t$

Sequence Name: \{Sequence Name\}

Run Time : :06-12-30 21:31:44

\section{Data Graph}

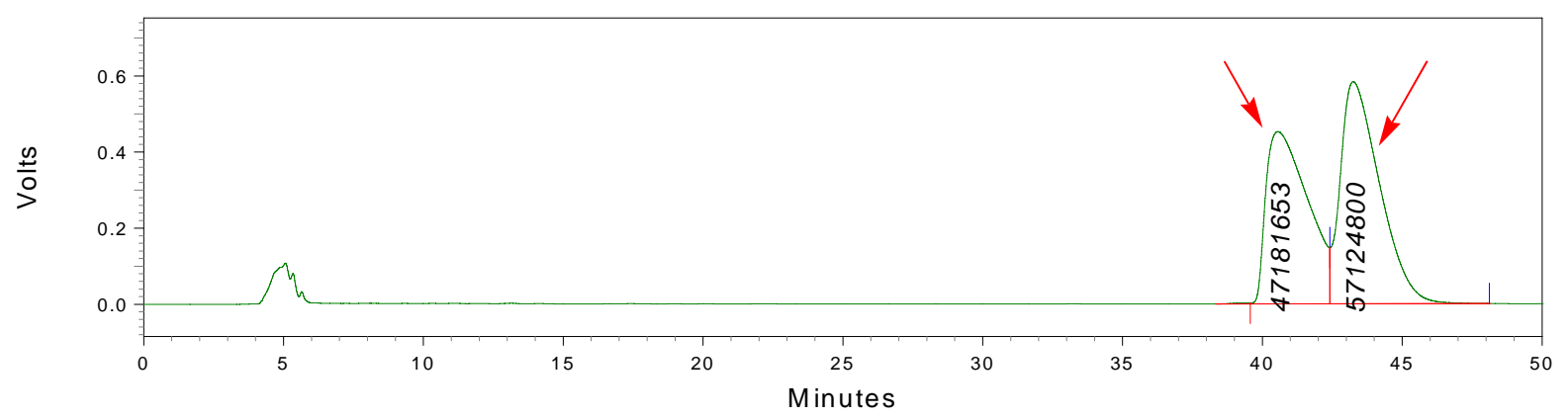

\section{Run Report}

Detector A $(254 \mathrm{~nm})$

Retention Time

Area

47181653

57124800

104306453
Area Percent

45.23

54.77

Totals 


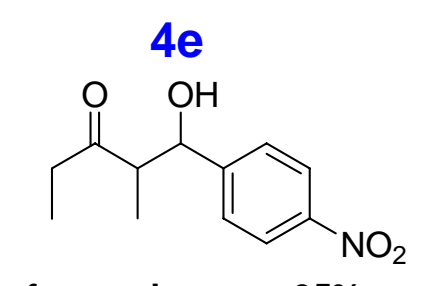

for syn isomer $>95 \%$ ee

Sample ID $\quad$ : As-H/0.8/20/254/5:95

User Name : System

Filename $\quad:$ E: $\backslash 429 \backslash x h \backslash 061230 \backslash 3$ wutong-ASH-1

Method Name : E:lgchl $\backslash$ m-4.met

Sequence Name: \{Sequence Name\}

Run Time : :06-12-30 22:32:03

\section{Data Graph}

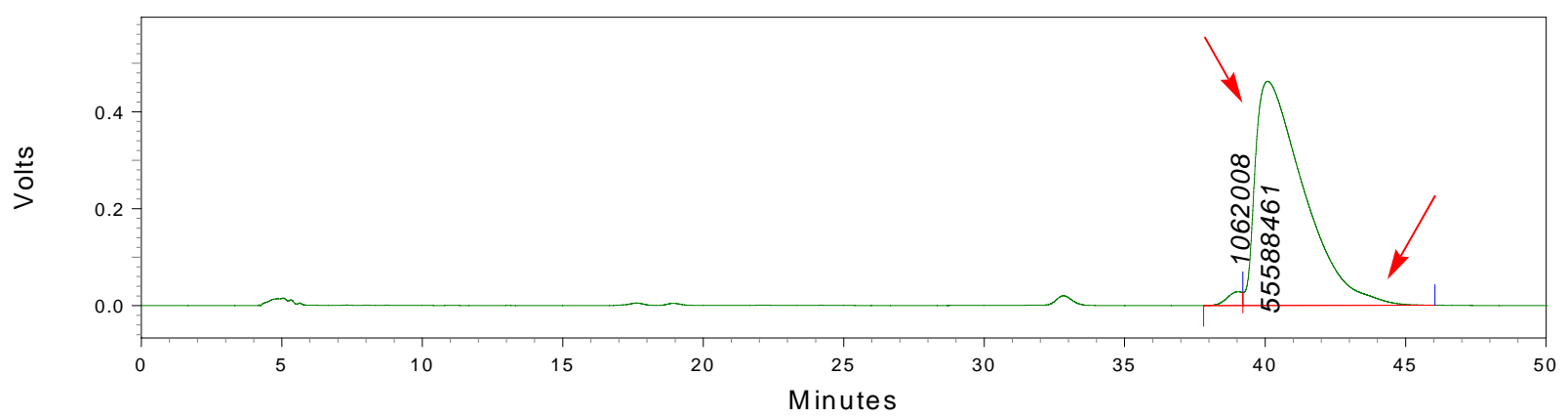

\section{Run Report}

Detector A (254nm)

Retention Time

Area

Area Percent

\begin{tabular}{|c|c|c|}
\multicolumn{1}{c}{ Retention Time } & Area & Area Percent \\
\hline 39.034 & 1062008 & 1.87 \\
40.085 & 55588461 & 98.13 \\
\hline Totals & & 100.00 \\
\hline
\end{tabular}




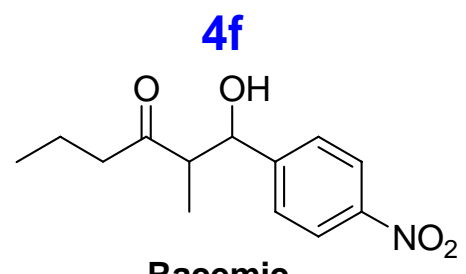

Sample ID : : AS-H/5:95/0.8/25/254

User Name : System

Filename : E: $429 \backslash x h \backslash 061220 \backslash$ racemic-3hexanone-2ed-final

Method Name : E: $\mid g c h l \backslash m-4$.met

Sequence Name: \{Sequence Name\}

Run Time : :06-12-21 7:46:51

\section{Data Graph}

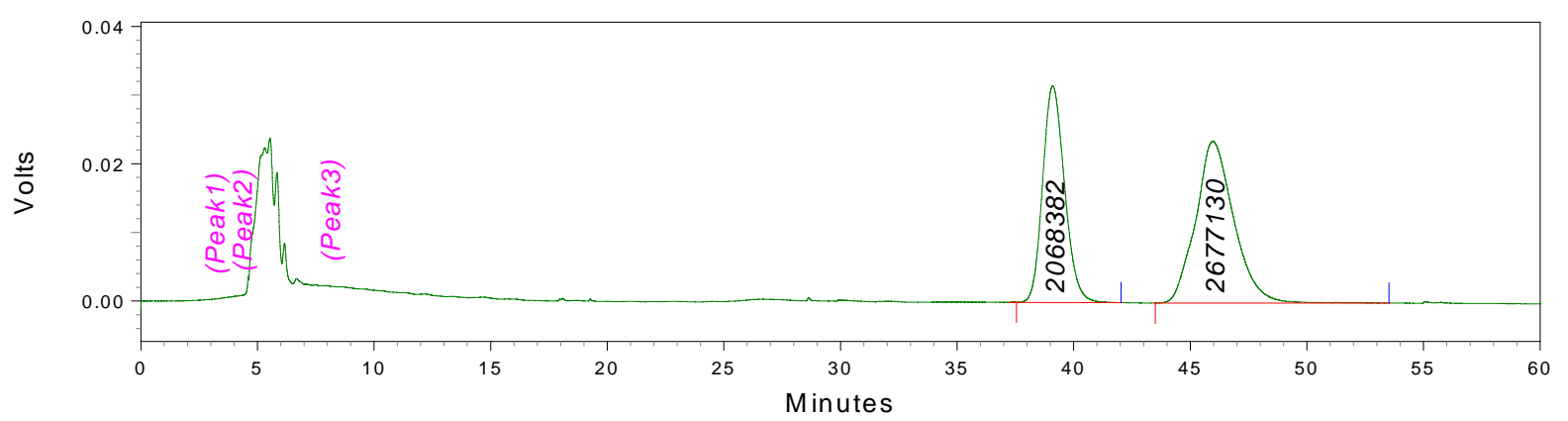

\section{Run Report}

\begin{tabular}{c|c|c|}
$\begin{array}{c}\text { Detector A }(254 \mathrm{~nm}) \\
\text { Retention Time }\end{array}$ & Area & Area Percent \\
\hline 39.097 & 2068382 & 43.59 \\
45.963 & 2677130 & 56.41 \\
\hline Totals & & 100.00 \\
\hline
\end{tabular}




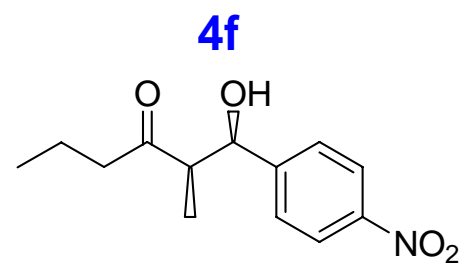

for syn isomer $96 \%$ ee

Sample ID : : AS-H/5:95/0.8/25/254

User Name : System

Filename $\quad$ : E: $\backslash 429 \backslash x h \backslash 061220 \backslash 3$ hexanone-sample1

Method Name : E:lgchl $\backslash$ m-4.met

Sequence Name: \{Sequence Name\}

Run Time : :06-12-21 6:05:06

\section{Data Graph}

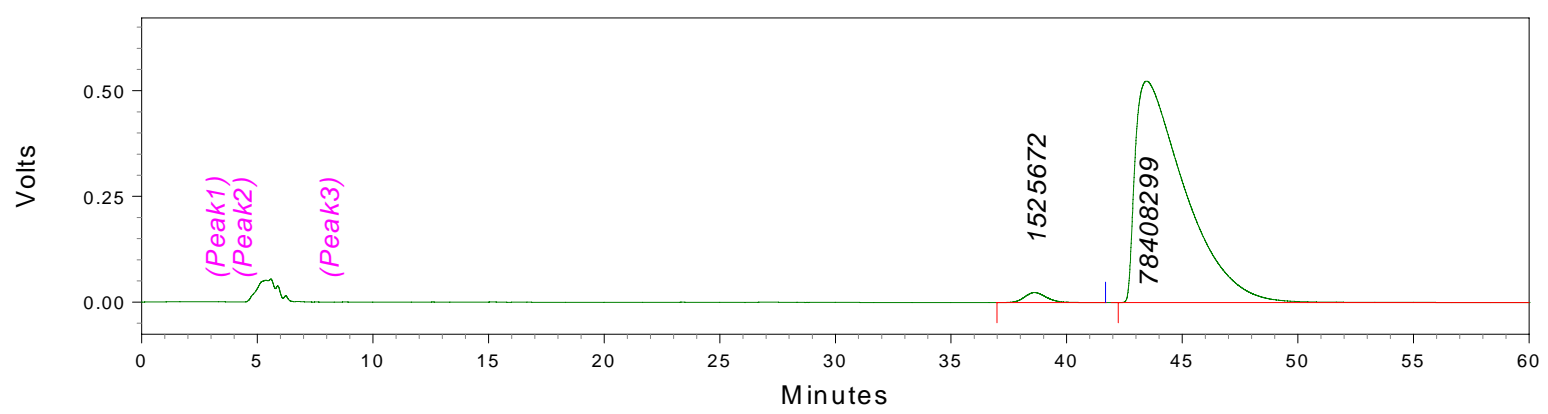

\section{Run Report}

Detector A (254nm)

Retention Time

Area

Area Percent

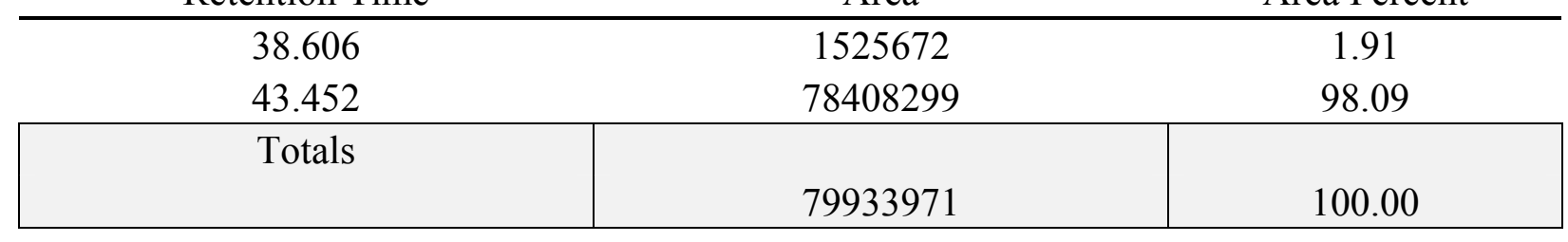




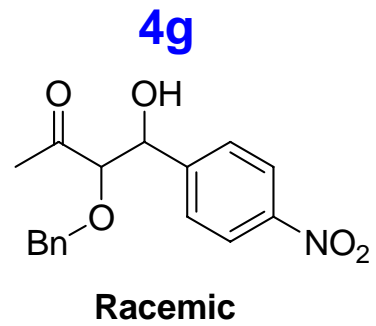
Sample ID : : AS-H/3:7/0.5/25/254
User Name : System
Filename : E: $1429 \backslash \mathrm{xh} \backslash 061227 \backslash \mathrm{racemic}-\mathrm{BnOacetone-pNO2-anti} \mathrm{and} \mathrm{syn}$
Method Name : E: $\mid g c h l \backslash m-4 . m e t$
Sequence Name: \{Sequence Name\}
Run Time : :06-12-27 20:34:47

\section{Data Graph}

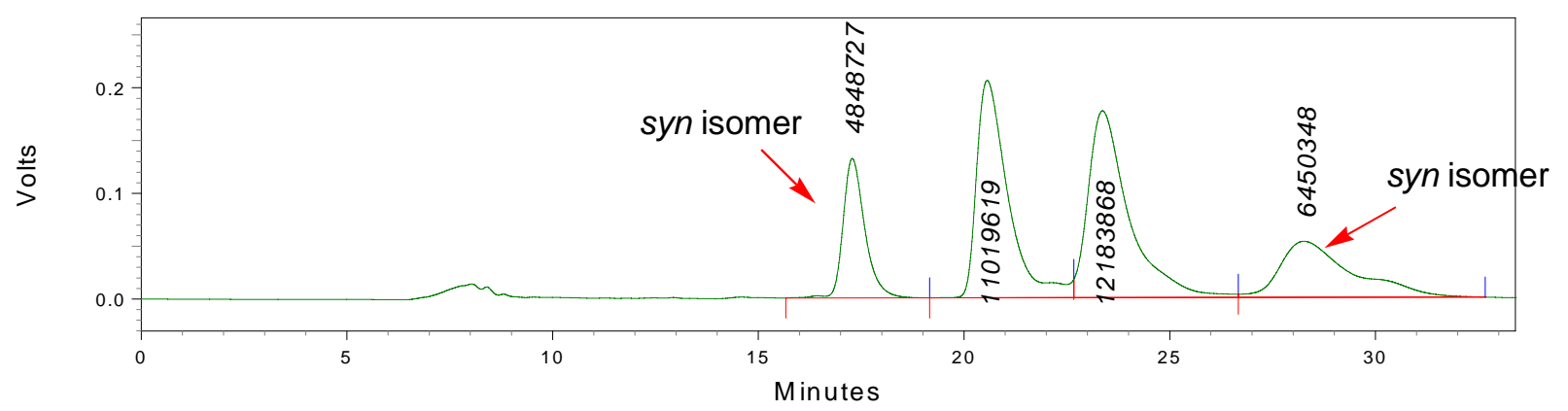

\section{Run Report}

Detector A $(254 \mathrm{~nm})$

\begin{tabular}{ccc} 
Retention Time & Area & Area Percent \\
\hline 17.287 & 4848727 & 14.05 \\
20.584 & 11019619 & 31.94 \\
23.375 & 12183868 & 35.31 \\
28.266 & 6450348 & 18.70 \\
\hline Totals & 34502561 & 100.00 \\
\hline
\end{tabular}




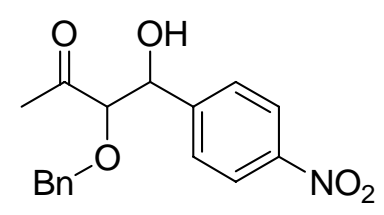

for syn isomer $97 \%$ ee
Sample ID : AS-H/3:7/0.5/25/254
User Name : System
Filename $\quad:$ E: $\backslash 429 \backslash x h \backslash 061227 \backslash$ BnOacetone-pNO2-syn-again-1
Method Name : E: Igchl $\backslash \mathrm{m}-4$.met

Sequence Name: \{Sequence Name\}

Run Time : :06-12-27 19:59:13

\section{Data Graph}

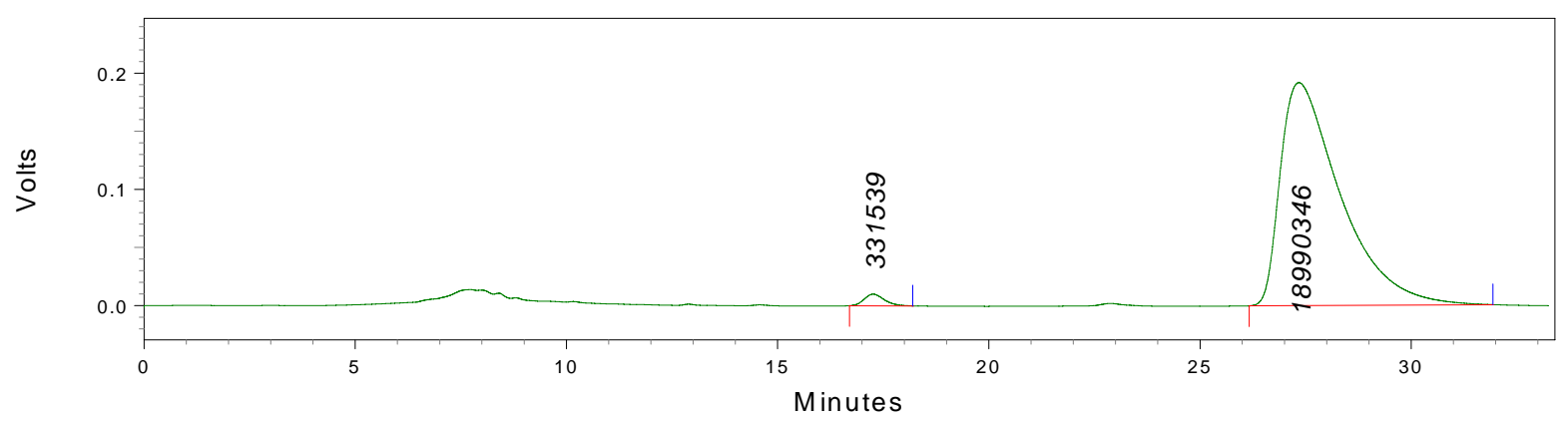

\section{Run Report}

\begin{tabular}{c|c|c|}
$\begin{array}{c}\text { Detector A }(254 \mathrm{~nm}) \\
\text { Retention Time }\end{array}$ & Area & Area Percent \\
\hline 17.258 & 331539 & 1.72 \\
27.337 & 18990346 & 98.28 \\
\hline Totals & 19321884 & 100.00 \\
\hline
\end{tabular}


<smiles>CC(=O)C(OCc1ccccc1)C(O)c1ccc(Cl)cc1</smiles>

Racemic

Acquired : 2007-2-7 13:39:29

Processed : 2007-2-7 15:03:37

Printed : 2007-2-8 12:14:58

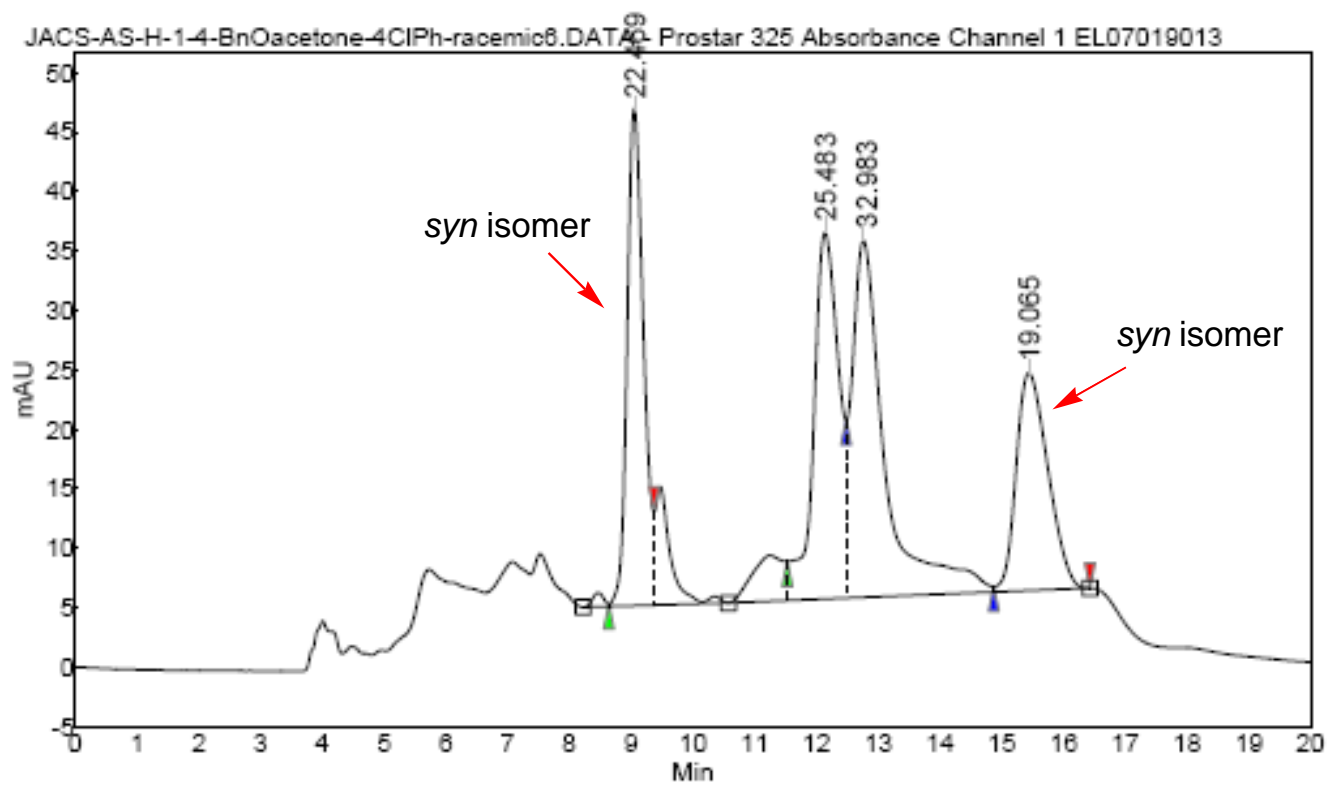

Peak results :

\begin{tabular}{|c|l|r|r|r|r|r|}
\hline Index & Name & $\begin{array}{r}\text { Time } \\
\text { [Min] }]\end{array}$ & $\begin{array}{r}\text { Quantity } \\
\text { [\% Area] }\end{array}$ & $\begin{array}{r}\text { Height } \\
\text { [mAU] }\end{array}$ & $\begin{array}{r}\text { Area } \\
\text { [mAU.Min] }\end{array}$ & $\begin{array}{r}\text { Area \% } \\
\text { [\%] }\end{array}$ \\
\hline \hline 1 & UNKNOWN & 9.05 & 22.47 & 41.8 & 12.8 & 22.469 \\
\hline 2 & UNKNOWN & 12.14 & 25.48 & 30.8 & 14.6 & 25.483 \\
\hline 3 & UNKNOWN & 12.76 & 32.98 & 30.0 & 18.8 & 32.983 \\
\hline 4 & UNKNOWN & 15.44 & 19.06 & 18.3 & 10.9 & 19.065 \\
\hline & & & & & & \\
\hline Total & & & 100.00 & 120.9 & 57.1 & 100.000 \\
\hline
\end{tabular}


Chromatogram : JACS-AS-H-1-4-BnOacetone-4CIPh-sample6_channel2

System : HPLC

Method : $\mathrm{XH}-1-4$

User : XH

Acquired : 2007-2-7 14:28:36

Processed : 2007-2-7 15:01:15

Printed : 2007-2-8 12:14:23<smiles>CC(=O)C(OCc1ccccc1)C(O)c1ccc(Cl)cc1</smiles>

for syn isomer $92 \%$ ee

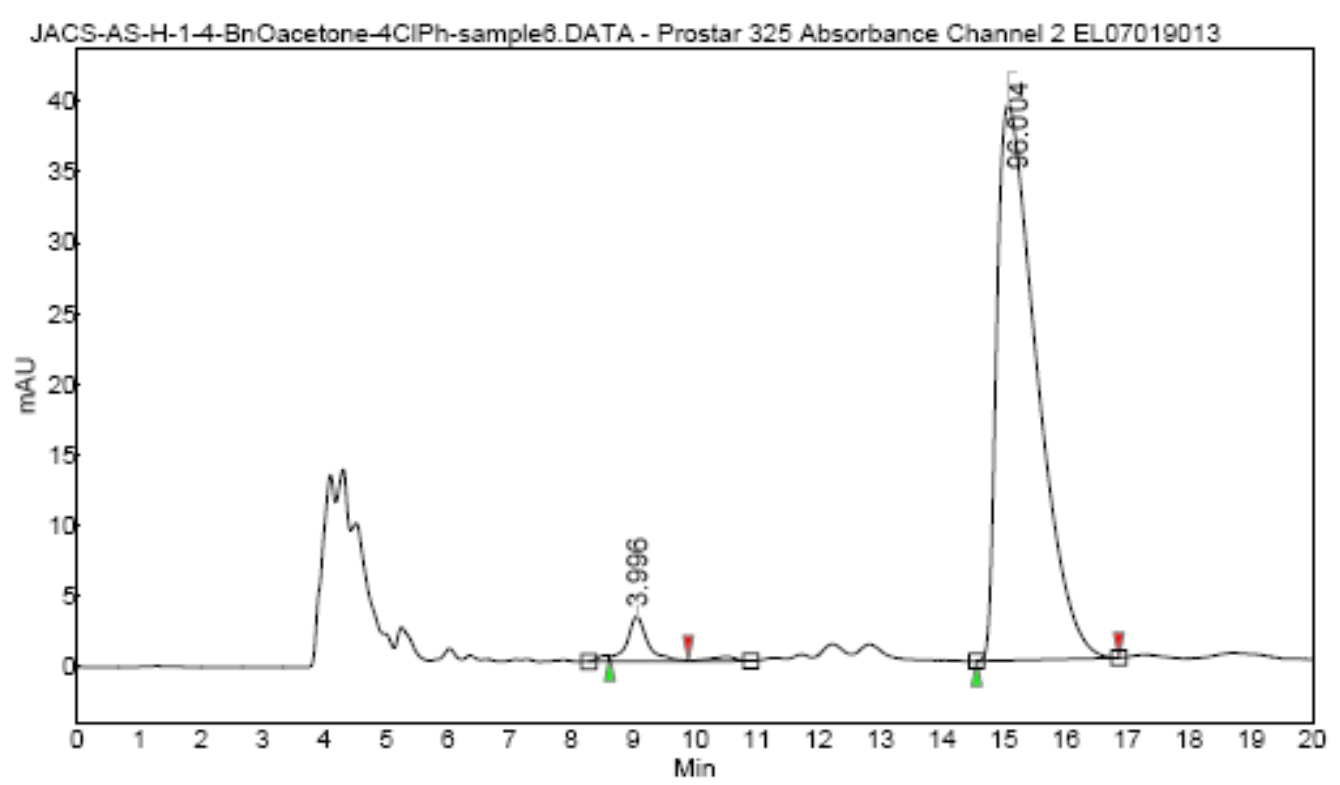

Peak results :

\begin{tabular}{|c|l|r|r|r|r|r|}
\hline Index & Name & $\begin{array}{r}\text { Time } \\
\text { [Min] }\end{array}$ & $\begin{array}{r}\text { Quantity } \\
\text { [\% Area] }\end{array}$ & $\begin{array}{r}\text { Height } \\
\text { [mAU] }\end{array}$ & $\begin{array}{r}\text { Area } \\
\text { [mAU.Min] }\end{array}$ & $\begin{array}{r}\text { Area \% } \\
\text { [\%] }\end{array}$ \\
\hline \hline 1 & UNKNOWN & 9.05 & 4.00 & 3.2 & 1.2 & 3.996 \\
\hline 2 & UNKNOWN & 15.07 & 96.00 & 39.3 & 29.3 & 96.004 \\
\hline & & & & & & \\
\hline Total & & & 100.00 & 42.5 & 30.5 & 100.000 \\
\hline
\end{tabular}


<smiles>CC(=O)C(OCc1ccccc1)C(O)c1cccc2ccccc12</smiles>

Racemic

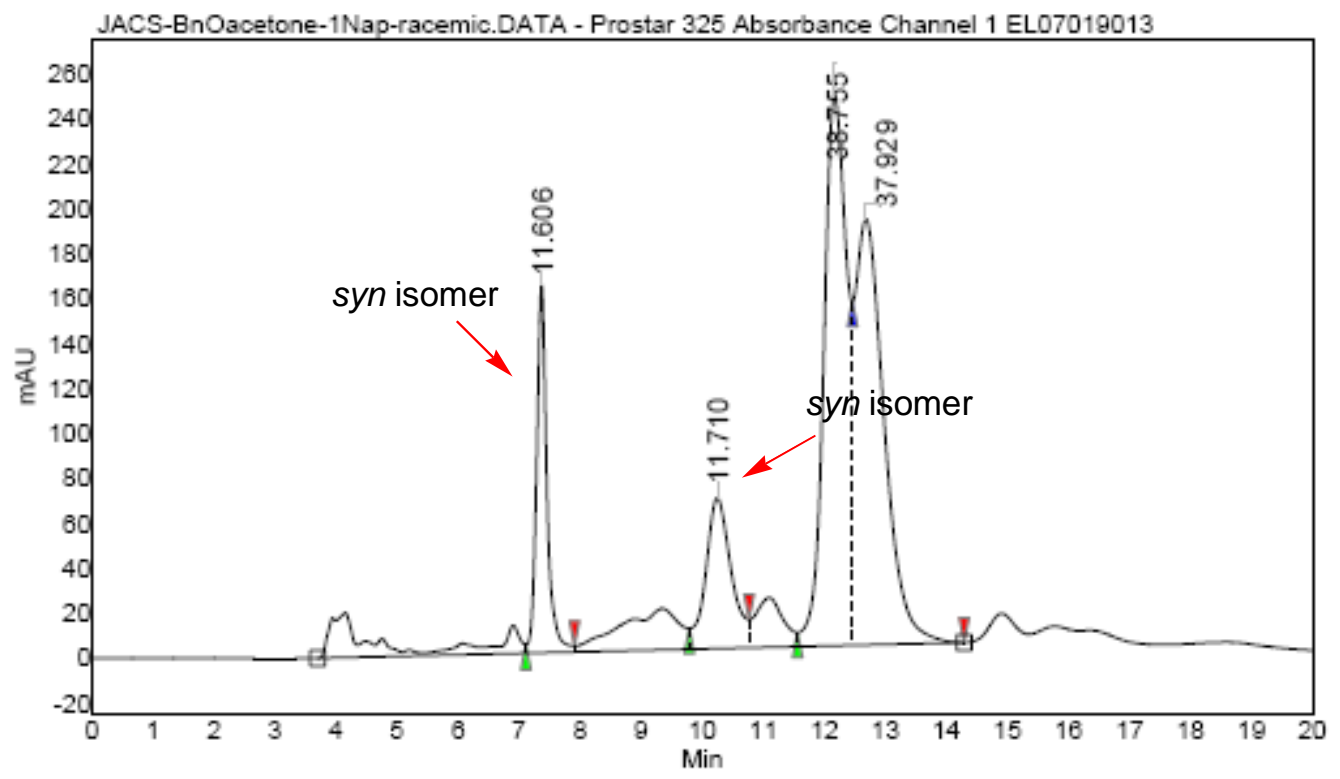

Peak results :

\begin{tabular}{|c|l|r|r|r|r|r|}
\hline Index & Name & $\begin{array}{r}\text { Time } \\
\text { [Min] }]\end{array}$ & $\begin{array}{r}\text { Quantity } \\
\text { [\% Area] }\end{array}$ & $\begin{array}{r}\text { Height } \\
\text { [mAU] }\end{array}$ & $\begin{array}{r}\text { Area } \\
\text { [mAU.Min] }\end{array}$ & $\begin{array}{r}\text { Area \% } \\
{[\%]}\end{array}$ \\
\hline \hline 1 & UNKNOWN & 7.37 & 11.61 & 163.7 & 32.2 & 11.606 \\
\hline 2 & UNKNOWN & 10.25 & 11.71 & 67.1 & 32.5 & 11.710 \\
\hline 3 & UNKNOWN & 12.17 & 38.75 & 244.9 & 107.7 & 38.755 \\
\hline 4 & UNKNOWN & 12.68 & 37.93 & 189.7 & 105.4 & 37.929 \\
\hline & & & & & & \\
\hline Total & & & 100.00 & 665.4 & 277.8 & 100.000 \\
\hline
\end{tabular}


System : HPLC

Method : $\mathrm{XH}-1-4$

User : $\mathrm{XH}$

Acquired : 2007-2-7 14:56:32

Processed : 2007-2-7 15:32:33

Printed : 2007-2-8 12:12:31<smiles>CC(=O)C(OCc1ccccc1)C(O)c1cccc2ccccc12</smiles>

for syn isomer $>99 \%$ ee

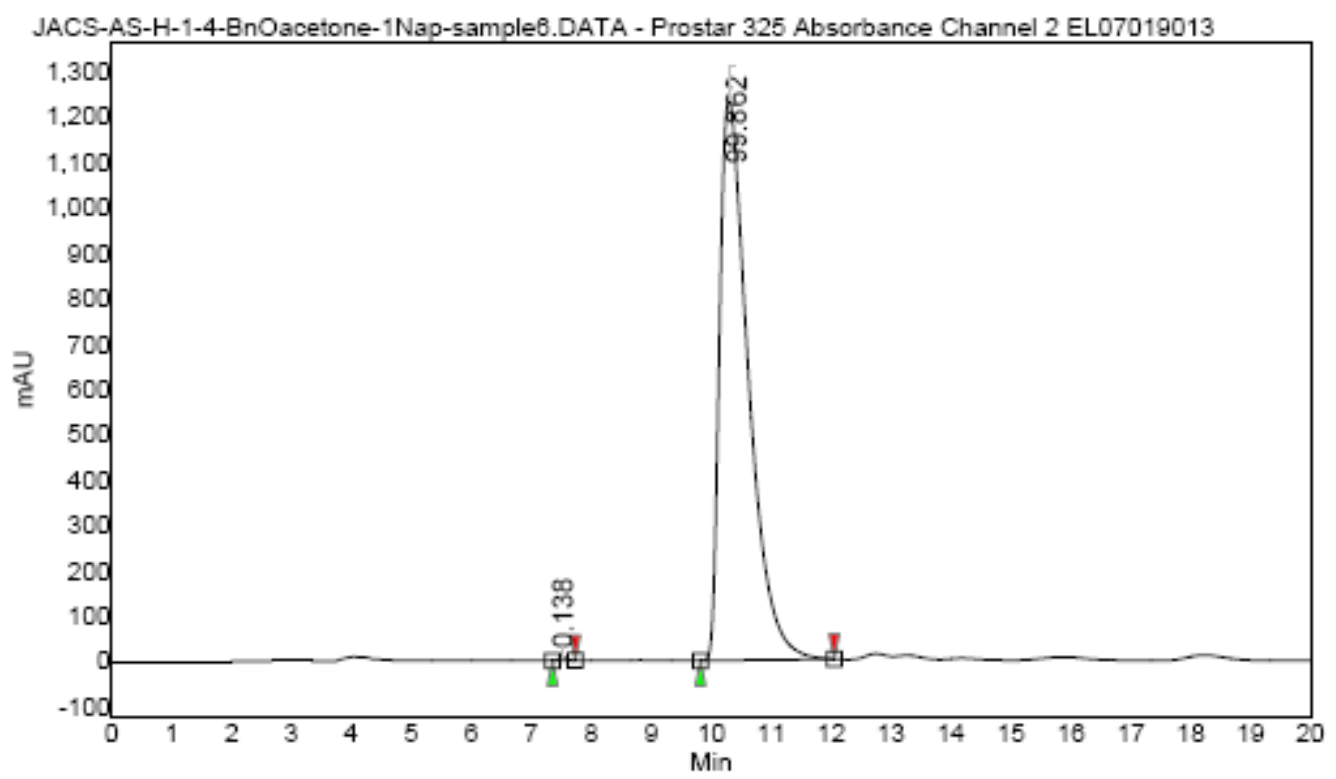

Peak results :

\begin{tabular}{|c|c|c|c|c|c|c|}
\hline Index & Name & $\begin{array}{l}\text { Time } \\
\text { [Min] }\end{array}$ & $\begin{array}{l}\text { Quantity } \\
\text { [\% Area] }\end{array}$ & $\begin{array}{l}\text { Height } \\
\text { [mAU] }\end{array}$ & $\begin{array}{r}\text { Area } \\
\text { [mAU.Min] }\end{array}$ & $\begin{array}{r}\text { Area \% } \\
{[\%]} \\
\end{array}$ \\
\hline 1 & UNKNOWN & 7.51 & 0.14 & 6.7 & 1.0 & 0.13 \\
\hline 2 & UNKNOWN & 10.30 & 99.86 & 1238.4 & 687.6 & 99.862 \\
\hline otal & & & 100.00 & 1245.1 & 688.6 & 10 \\
\hline
\end{tabular}


Chromatogram : JACS-AS-H-1-4-BnOacetone-Ph-racemic-again-2-8_channel1

System : HPLC

Method : $\mathrm{XH}-1-4$

User : $\mathrm{XH}$

Acquired : 2007-2-8 11:07:11

Processed : 2007-2-8 12:09:35

Printed : 2007-2-8 12-09:38<smiles>CC(=O)C(OCc1ccccc1)C(O)c1ccccc1</smiles>

Racemic

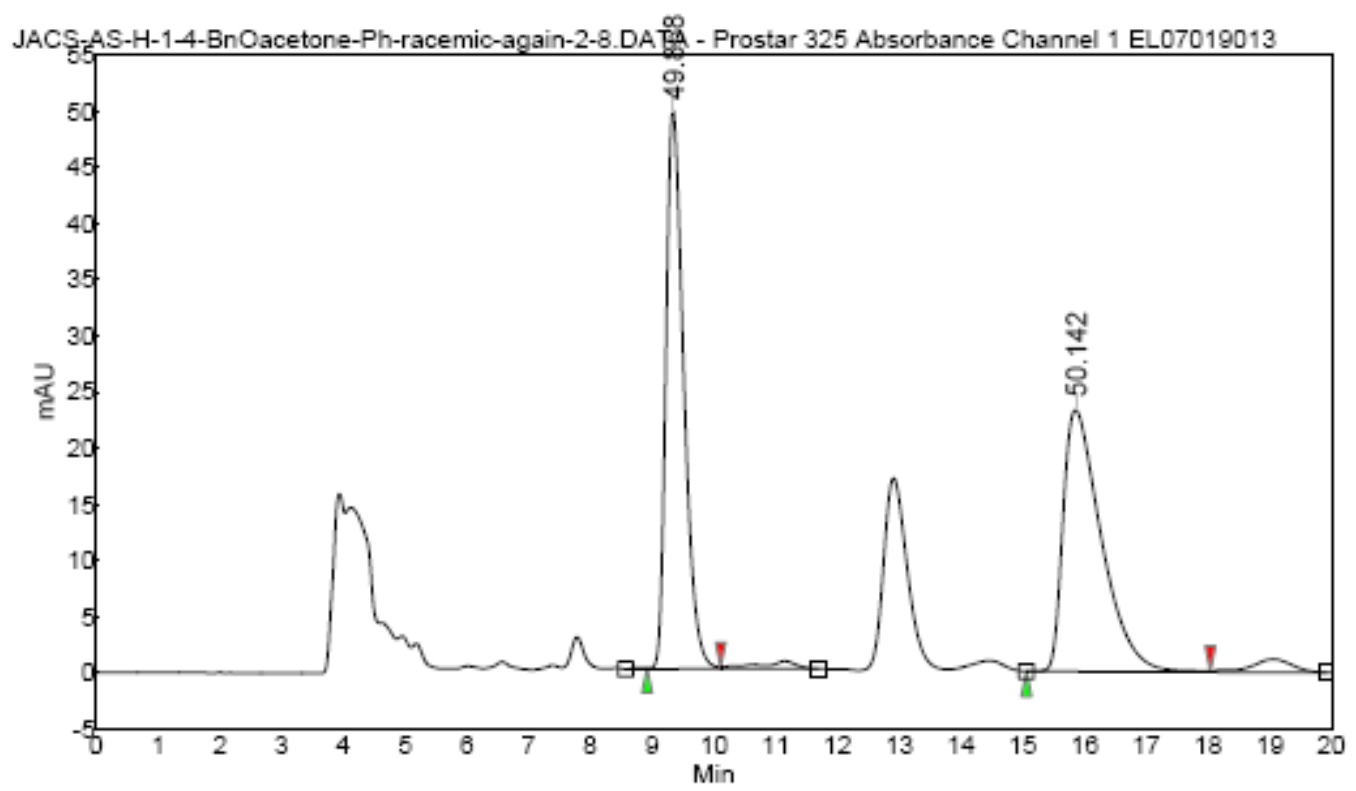

Peak results :

\begin{tabular}{|c|l|r|r|r|r|r|}
\hline Index & Name & $\begin{array}{r}\text { Time } \\
\text { [Min] }\end{array}$ & $\begin{array}{r}\text { Quantity } \\
\text { [\% Area] }\end{array}$ & $\begin{array}{r}\text { Height } \\
\text { [mAU] }\end{array}$ & $\begin{array}{r}\text { Area } \\
\text { [mAU.Min] }\end{array}$ & $\begin{array}{r}\text { Area \% } \\
\text { [\%] }\end{array}$ \\
\hline \hline 1 & UNKNOWN & 9.34 & 49.86 & 49.8 & 16.6 & 49.858 \\
\hline 2 & UNKNOWN & 15.86 & 50.14 & 23.3 & 16.7 & 50.142 \\
\hline & & & & & & \\
\hline Total & & & 100.00 & 73.1 & 33.3 & 100.000 \\
\hline
\end{tabular}


<smiles>CC(=O)C(OCc1ccccc1)C(O)c1ccccc1</smiles>

for syn isomer $86 \%$ ee

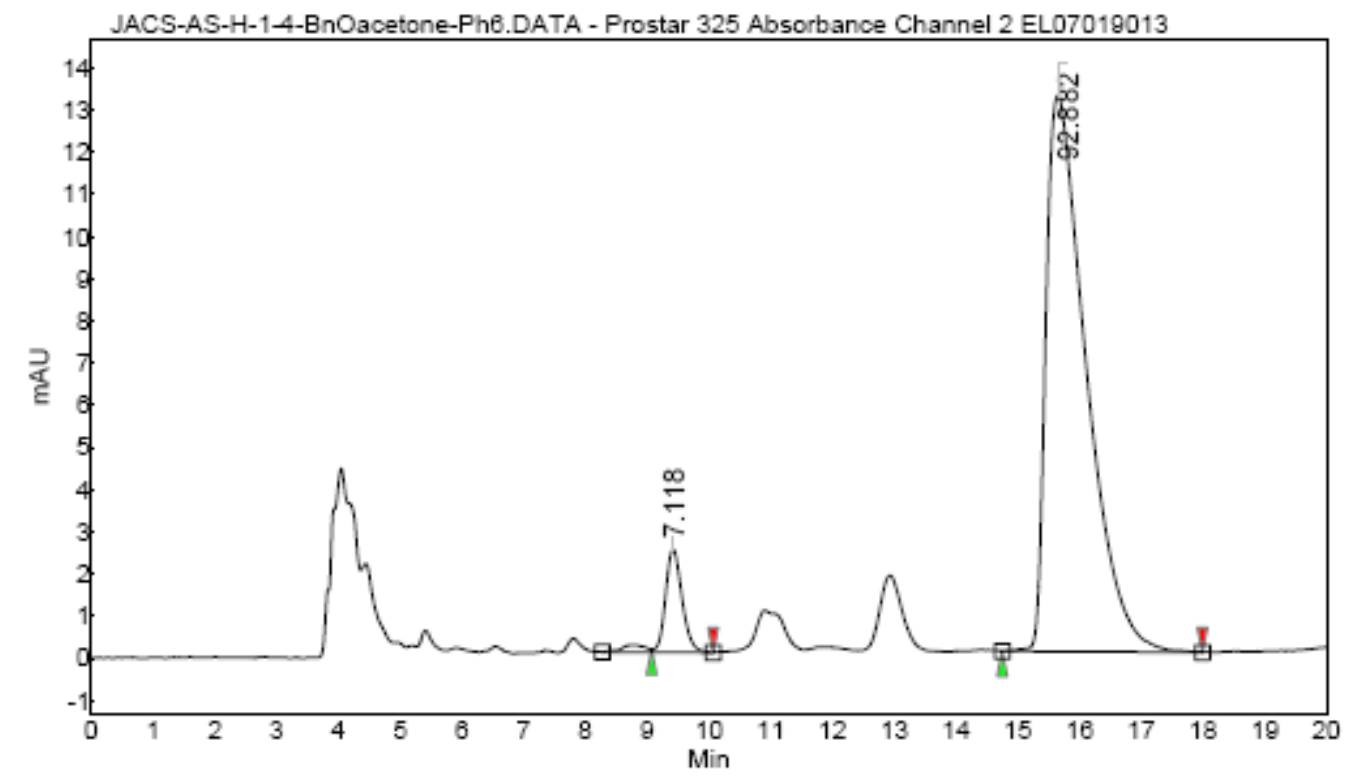

Peak results :

\begin{tabular}{|c|l|r|r|r|r|r|}
\hline Index & Name & $\begin{array}{r}\text { Time } \\
\text { [Min] }\end{array}$ & $\begin{array}{r}\text { Quantity } \\
\text { [\% Area] }\end{array}$ & $\begin{array}{r}\text { Height } \\
\text { [mAU] }\end{array}$ & $\begin{array}{r}\text { Area } \\
\text { [mAU.Min] }\end{array}$ & $\begin{array}{r}\text { Area \% } \\
\text { [\%] }\end{array}$ \\
\hline \hline 1 & UNKNOWN & 9.42 & 7.12 & 2.5 & 0.8 & 7.118 \\
\hline 2 & UNKNOWN & 15.65 & 92.88 & 13.2 & 9.9 & 92.882 \\
\hline & & & & & & \\
\hline Total & & & 100.00 & 15.6 & 10.7 & 100.000 \\
\hline
\end{tabular}




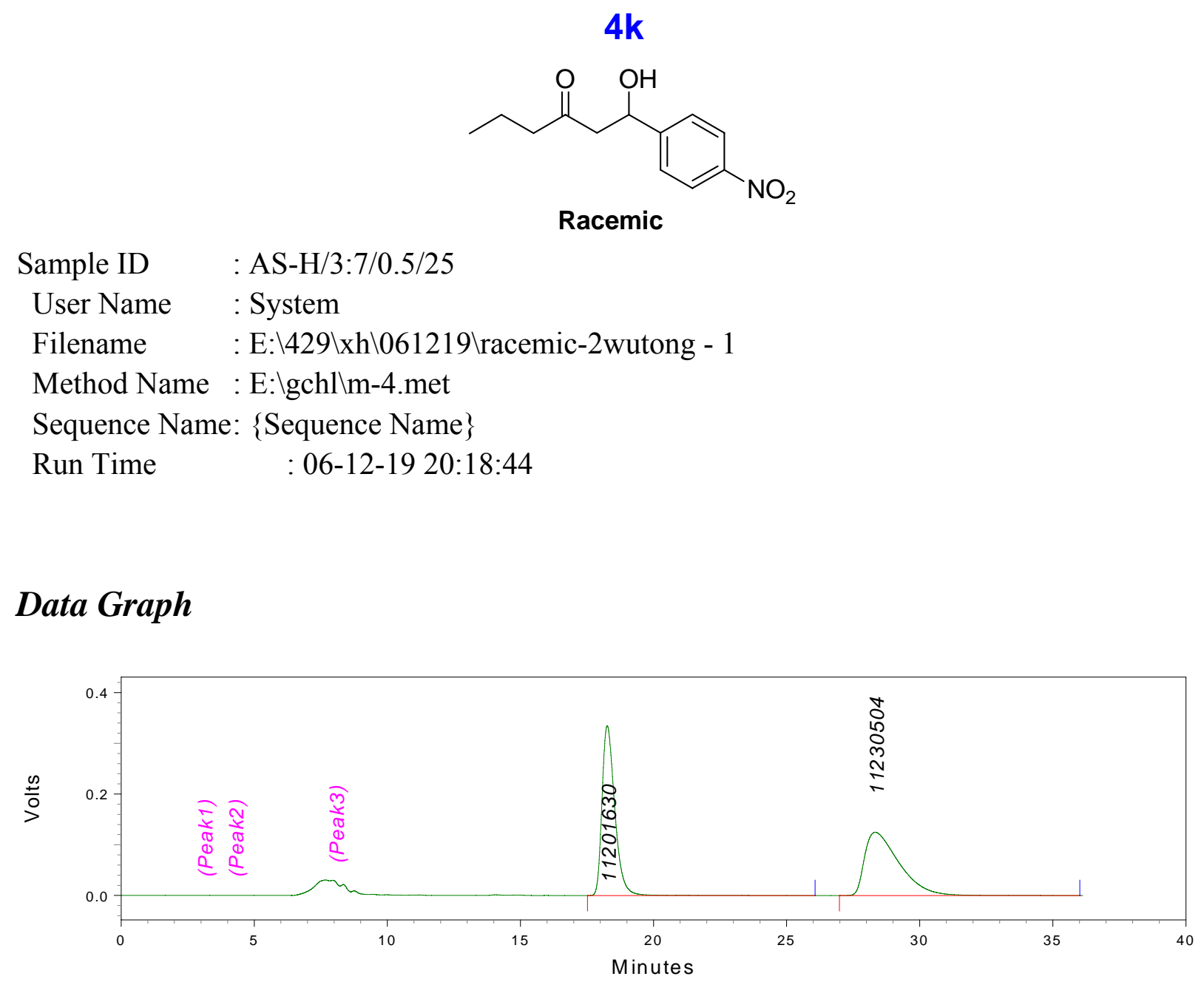

\section{Run Report}

Detector A (254nm)

\begin{tabular}{|ccc|} 
Retention Time & Area & Area Percent \\
\hline 18.255 & 11201630 & 49.94 \\
28.325 & 11230504 & 50.06 \\
\hline Totals & 22432134 & 100.00 \\
\hline
\end{tabular}




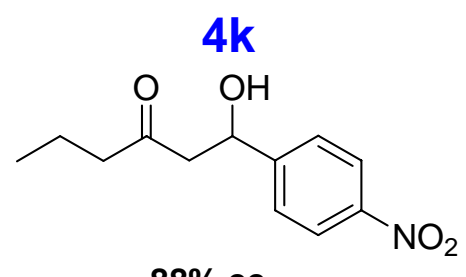

Sample ID $\quad$ : AS-H/3:7/0.5/25

User Name : System

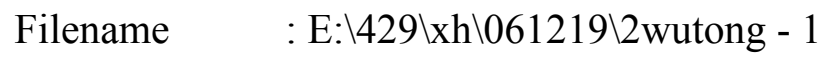

Method Name : E: $\mid g c h l \backslash m-4 . m e t$

Sequence Name: \{Sequence Name\}

Run Time : : 06-12-19 19:00:53

\section{Data Graph}

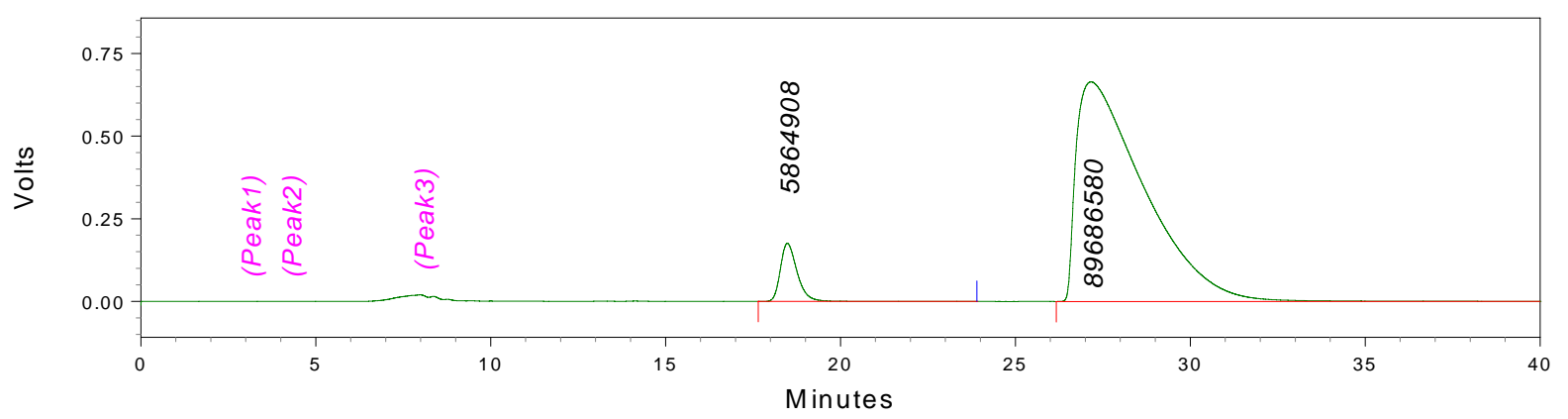

\section{Run Report}

Detector A (254nm)

Retention Time

Area

Area Percent

\begin{tabular}{|c|c|c|}
\hline \multicolumn{1}{c}{ Area } & Area Percent \\
\hline 18.478 & 5864908 & 6.14 \\
27.158 & 89686580 & 93.86 \\
\hline Totals & & 100.00 \\
\hline
\end{tabular}




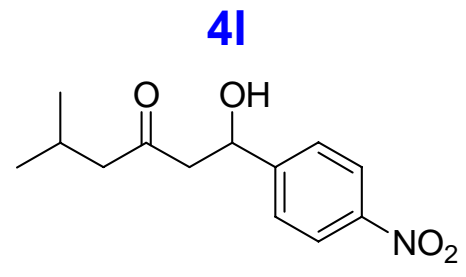

Racemic

Sample ID : : AS-H/3:7/25/0.6/254

User Name : System

Filename $\quad$ : E: $\backslash 429 \backslash x h \backslash 061217 \backslash$ racemic-4methyl-wutong

Method Name : E: $\operatorname{lgchl} \backslash \mathrm{m}-4$.met

Sequence Name: \{Sequence Name\}

Run Time $\quad$ :06-12-17 13:28:14

\section{Run}

\section{Report}

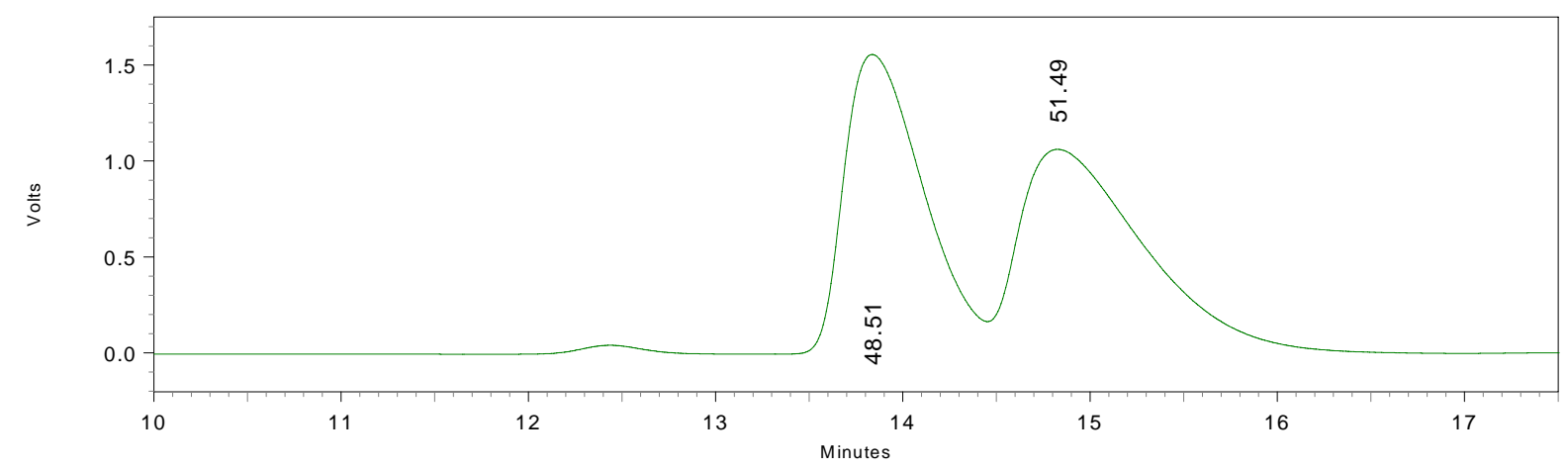

Detector A (254nm)

Retention Time Area

Area Percent

13.832
14.823

45601467

48.51

14.823

48403851

51.49

Totals 


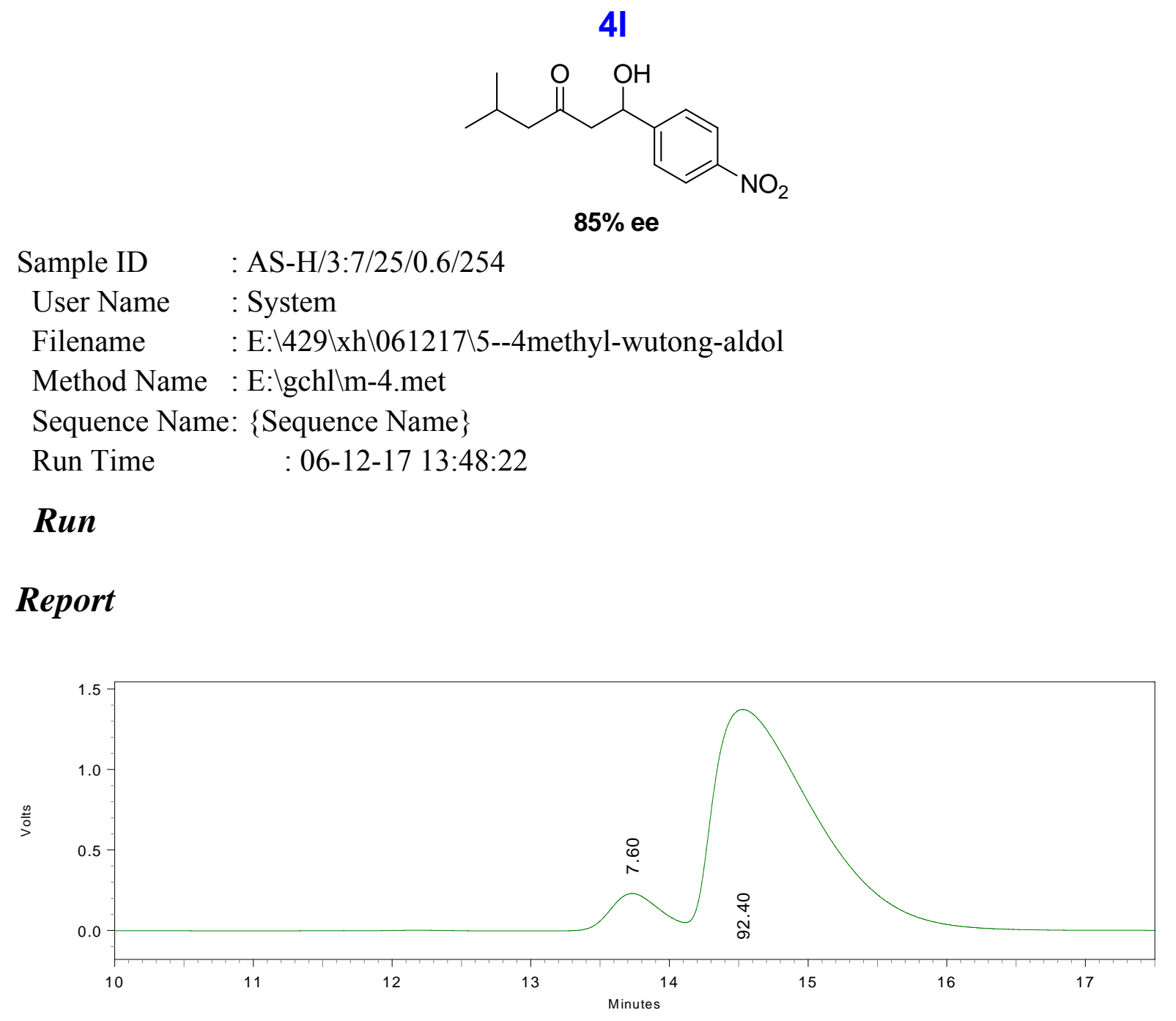

Detector A (254nm)

Retention Time

Area

Area Percent

\begin{tabular}{|c|c|c|}
\multicolumn{1}{c}{ Retention Time } & Area & Area Percent \\
\hline 13.727 & 5615281 & 7.60 \\
14.524 & 68274932 & 92.40 \\
\hline Totals & & 100.00 \\
\hline
\end{tabular}




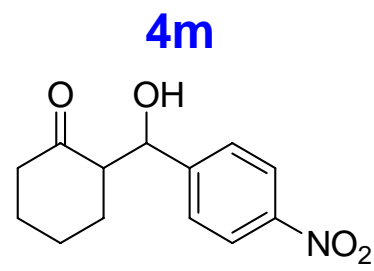

Racemic

Sample ID : AD-H/1:4/0.5/25/254

User Name : System

Filename $\quad: \mathrm{E}: \backslash 429 \backslash \mathrm{xh} \backslash 061227 \backslash$ racemic-cyclohexanone-pNO2-anti

Method Name : E: Igchl \m-4.met

Sequence Name: \{Sequence Name\}

Run Time : :06-12-27 10:55:24

\section{Data Graph}

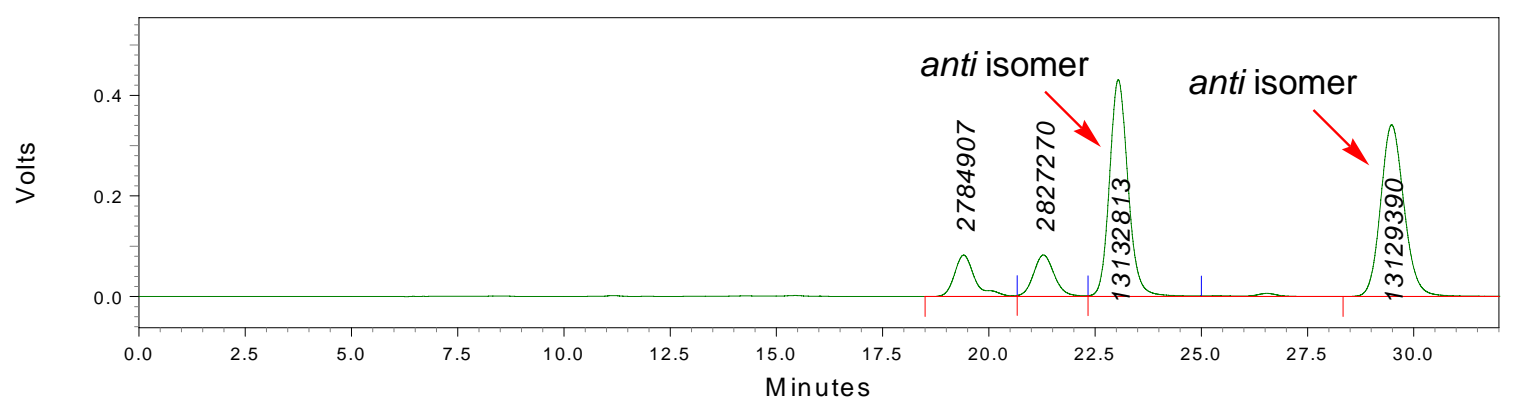

\section{Run Report}

Detector A (254nm)

Retention Time

Area

2784907

2827270

13132813

13129390

31874379
Area Percent

8.74

8.87

41.20

29.474

Totals
41.19

100.00 


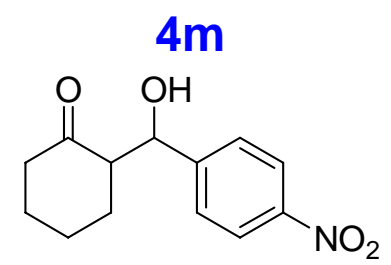

for anti isomer $98 \%$ ee

Sample ID $\quad:$ AD-H-0.5-25-1:4

User Name : System

Filename $\quad$ : E: $\backslash 429 \backslash \mathrm{xh} \backslash 060825 \backslash 1-\mathrm{CH} 2 \mathrm{Cl} 2$

Method Name : E: $\operatorname{lgchl} \backslash \mathrm{m}-4$.met

Sequence Name: \{Sequence Name\}

Run Time : :06-8-25 15:50:51

\section{Data Graph}

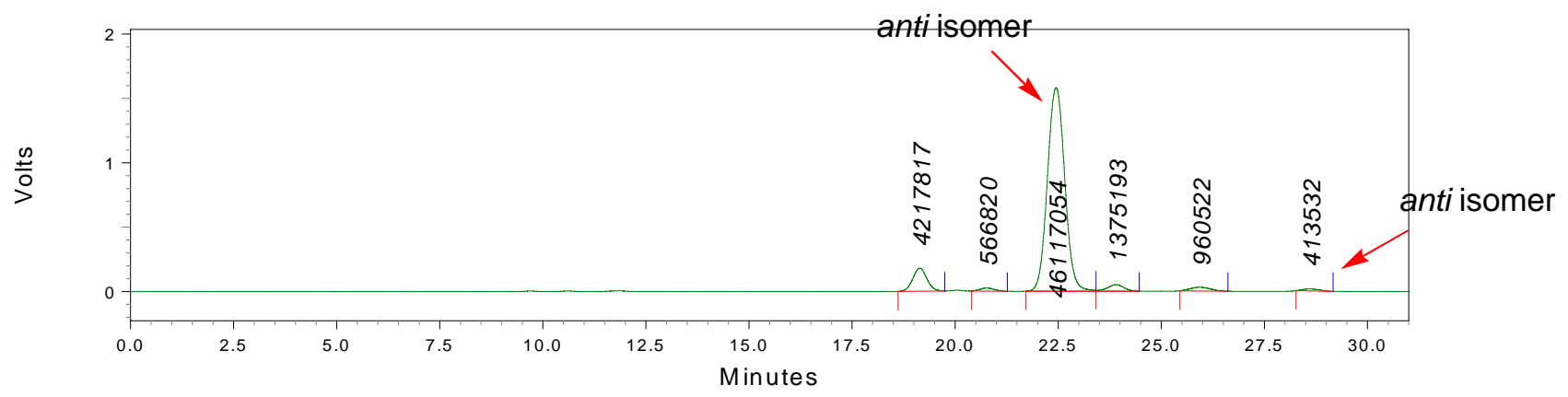

\section{Run Report}

Detector A $(254 \mathrm{~nm})$

\begin{tabular}{ccc} 
Retention Time & Area & Area Percent \\
\hline 19.136 & 4217817 & 7.86 \\
20.760 & 566820 & 1.06 \\
22.443 & 46117054 & 85.96 \\
23.898 & 1375193 & 2.56 \\
25.928 & 960522 & 1.79 \\
28.598 & 413532 & 0.77 \\
\hline Totals & & 100.00 \\
\hline
\end{tabular}

